\title{
In vivo super-resolution live-cell RESOLFT-microscopy of Drosophila melanogaster and Arabidopsis thaliana
}

\author{
Dissertation \\ for the award of the degree \\ "Doctor rerum naturalium" \\ of the Georg-August-Universität Göttingen
}

within the doctoral program in Biology

of the Georg-August University School of Science (GAUSS)

submitted by

Sebastian Schnorrenberg

from Hürth

Göttingen, 2017 
Thesis committee:

Prof. Dr. Stefan Jakobs

Department of NanoBiophotonik

Max Planck Institute for Biophysical Chemistry

PD Dr. Halyna Shcherbata

Max Planck Research Group for Gene Expression and Signaling

Max Planck Institute for Biophysical Chemistry

Members of the examination board:

Reviewer:

Prof. Dr. Stefan Jakobs

Department of NanoBiophotonik

Max Planck Institute for Biophysical Chemistry

Second reviewer:

PD Dr. Halyna Shcherbata

Max Planck Research Group for Gene Expression and Signaling

Max Planck Institute for Biophysical Chemistry

Further members of the Examination board:

Prof. Dr. Dr. h. c. mult. Stefan Hell

Department of NanoBiophotonik

Max Planck Institute for Biophysical Chemistry

Dr. Melina Schuh

Department of Meiosis

Max Planck Institute for Biophysical Chemistry

PD Dr. Thomas Teichmann

Department of Plant Cell Biology

Georg-August-University Goettingen

Dr. Gerd Vorbrüggen

Developmental Biology

Georg-August-University Goettingen

Date of oral examination: $15^{\text {th }}$ August, 2017 


\section{Affidavit}

I hereby declare that my doctoral thesis entitled "In vivo super-resolution live-cell RESOLFT-microscopy of Drosophila melanogaster and Arabidopsis thaliana" has been written independently and with no other sources and aids than quoted. I have indicated the parts which were performed by project collaborators.

Sebastian Schnorrenberg

Göttingen, July 2017 


\section{Abstract}

The optical resolution of conventional light microscopy is limited by the diffraction barrier of light. This resolution limit impedes the characterization of cellular structures featuring a size smaller than half the wavelength of light. RESOLFT (reversible saturable optical linear fluorescence transitions)-microscopy breaks the diffraction barrier by targeted swichting of fluorophores between separable states using low light levels. Since its first demonstration in 2005, RESOLFT-microscopy has proved its usability in high resolution imaging of living single cells.

This work reports, for the first time, the establishment of RESOLFT microscopy in a multicellular organism, namely Drosophila melanogaster and its first application for in vivo-imaging.

Using RESOLFT-microscopy on isolated tissues, a lateral resolution of $50-60 \mathrm{~nm}$ on single microtubule filaments was achieved. Additionally, the dynamic changes of the microtubule network were captured in high spatial resolution by recording 40 consecutive RESOLFT frames. The fluorescent probe rsEGFP2 enabled high resolution three dimensional imaging with 4 fold improved axial resolution. Using intact living Drosophila melanogaster larvae, in vivo high resolution RESOLFT imaging with a resolution of $\sim 50 \mathrm{~nm}$ could be demonstrated, which up to now had not been shown.

Moreover, this work reports on the first application of RESOLFT-microscopy in the plant Arabidopsis thaliana. The relatively low light levels used in RESOLFTmicroscopy enabled high resolution imaging in this light absorbing model organism of plant research. RESOLFT imaging of green leaf epidermis cells resulted in lateral resolution of $\sim 70 \mathrm{~nm}$. Further, it could be shown that the autofluorescence of chloroplasts can be excluded in the fluorescence recordings of rsEGFP2 using fluorescence lifetime gating. Time-lapse recording facilitated the characterization of the dynamic behaviour of microtubule filaments in epidermis cells over a period of approximately 1 hour without any signs of phototoxicity. 


\section{Acronyms}

\begin{tabular}{|c|c|}
\hline $3 \mathrm{D}$ & three-dimensional \\
\hline CCD & charge-coupled device \\
\hline CSLM & confocal laser scanning microscopy \\
\hline $\mathrm{CW}$ & continous wave \\
\hline DNA & deoxyribonucleic acid \\
\hline EGTA & ethylene glycol-bis( $\beta$-aminoethyl ether)-N,N,N',N'-tetraacetic acid \\
\hline FBS & fetal bovine serum \\
\hline FRAP & fluorescence recovery after photobleaching \\
\hline FWHM & full width at half maximum \\
\hline GFP & green fluorescent protein \\
\hline GSD & ground state depletion \\
\hline HEPES & (4-(2-hydroxyethyl)-1-piperazineethanesulfonic acid) \\
\hline $\mathrm{HRP}$ & horseradish peroxidase \\
\hline NIR & near infrared \\
\hline MAP & microtubule-associated protein \\
\hline NA & numerical aperture \\
\hline PALM & photoactivatable localization microscopy \\
\hline PBS & phosphate buffered saline \\
\hline $\mathrm{PSF}$ & point spread function \\
\hline PVDF & polyvinylidene difluoride \\
\hline RESOLFT & reversible saturable optical fluorescence transition \\
\hline RSFP & reversibly switchable fluorescent protein \\
\hline SDS & sodium dodecyl sulfate \\
\hline SIM & structured illumination microscopy \\
\hline SLM & spatial light modulator \\
\hline SSIM & saturated structured illumination microscopy \\
\hline STED & stimulated emission depletion \\
\hline STORM & stochastic optical reconstruction microscopy \\
\hline TIRF & total internal reflection fluorescence \\
\hline UV & ultraviolet \\
\hline
\end{tabular}




\section{Contents}

1 Introduction $\quad 1$

1.1 Fluorescence microscopy . . . . . . . . . . . . . . . . . . . 1

1.1.1 Fluorescence . . . . . . . . . . . . . . . . . . 1

1.1 .2 Fluorescence microscopy . . . . . . . . . . . . . 2

1.2 Theoretical resolution of a light-microscope . . . . . . . . . . . . . . . 4

1.3 Breaking the diffraction-barrier of light microscopy . . . . . . . . . 6

1.3.1 Coordinated-stochastic methods . . . . . . . . . . . . . 6

1.3.2 Coordinated-targeted methods . . . . . . . . . . . . . 8

1.3.2.1 RESOLFT-type microscopy techniques . . . . . . . . 10

1.4 Reversibly switchable fluorescent proteins . . . . . . . . . . . . . . . . 11

1.4.1 Photophysical properties of RSFPs . . . . . . . . . . . . 11

1.4.2 Switching mechanism of RSFPs . . . . . . . . . . . . . . . 13

1.4.3 RSFPs used for RESOLFT-imaging . . . . . . . . . . . . . . . 15

1.4.4 Point scanning RESOLFT-microscopy using RSFPs . . . . . . 18

1.5 Nanoscopy of Drosophila melanogaster . . . . . . . . . . . . . . . . . 19

1.6 Nanoscopy of Arabidopsis thaliana . . . . . . . . . . . . . . . . . . . . 21

1.7 Aim of the study . . . . . . . . . . . . . . . . . . . . 23

2 Results $\quad 24$

2.1 RESOLFT-microscopy of Drosophila melanogaster . . . . . . . . . . . 24

2.1.1 Generation of stably expressing rsEGFP2- $\alpha$-tubulin Drosophila melanogaster . . . . . . . . . . . . . . 24

2.1.2 Characterisation of rsEGFP2- $\alpha$-tubulin expression . . . . . . . 24

2.1.3 RESOLFT-microscopy of dissected tissues . . . . . . . . . . . 27

2.1.3.1 2D-RESOLFT imaging of living tissues . . . . . . . 27

2.1.3.2 3D-RESOLFT imaging of living tissues . . . . . . . . 31

2.1.4 RESOLFT-microscopy at different imaging depths . . . . . . . 33

2.1.5 Time-lapse RESOLFT-imaging . . . . . . . . . . . . 36

2.1.6 In vivo RESOLFT-microscopy of intact larva . . . . . . . . . . 39

2.2 High-resolution RESOLFT-microscopy of Arabidopsis thaliana . . . . 43

2.2.1 Generation of rsEGFP2 expressing Arabidopsis thaliana . . . . 43

2.2.2 Characterisation of MAP4-rsEGFP2 expression pattern in Arabidopsis thaliana . . . . . . . . . . . . . 43

2.2.3 Autofluorescence suppression by fluorescence lifetime gating . 45

2.2.4 RESOLFT-microscopy of Arabidopsis thaliana . . . . . . . . . 46 
2.2.5 STED-microscopy of MAP4-rsEGFP2 labelled microtubule filaments in leaf epidermis cells . . . . . . . . . . . 48

2.2.6 RESOLFT time-lapse imaging of Arabidopsis thaliana . . . . . 50

2.2.7 Bleaching analysis of rsEGFP2 in Arabidopsis thaliana . . . . 52 2.2.7.1 Bleaching analysis . . . . . . . . . . . 52

2.2.8 FRAP measurements on MAP4-rsEGFP2 labelled microtubule filaments . . . . . . . . . . . . . . . . . 54

2.2.9 Filament counting in RESOLFT time-lapse series . . . . . . 55

3 Discussion $\quad 58$

3.1 RESOLFT-microscopy of Drosophila melanogaster . . . . . . . . . . . 58

3.1.1 rsEGFP2- $\alpha$-tubulin expressing Drosophila melanogaster . . . . 58

3.1.2 Optical resolution in RESOLFT imaging of Drosophila melanogaster ..................... 59

3.1.3 Photostress in RESOLFT-microscopy . . . . . . . . . . . . . . 61

3.1.4 Tissue RESOLFT-microscopy . . . . . . . . . . . . . . . 62

3.1 .5 In vivo RESOLFT-microscopy . . . . . . . . . . . . . . . . . . 64

3.1.6 Conclusions and Outlook . . . . . . . . . . . . . 65

3.2 RESOLFT-microscopy of Arabidopsis thaliana . . . . . . . . . . . . 66

3.2.1 High-resolution imaging of Arabidopsis thaliana . . . . . . . . 66

3.2.2 Labelling of microtubule network by MAP4-rsEGFP2 expression 67

3.2.3 Autofluorescence suppression by fluorescence lifetime gating . 68

3.2.4 Live-cell STED-microscopy of Arabidopsis thaliana . . . . . . 69

3.2.5 Time-lapse RESOLFT recordings . . . . . . . . . . . . 70

3.2.6 FRAP measurements of MAP4-rsEGFP2 labelled microtubules 71

3.2.7 Conclusions and Outlook . . . . . . . . . . . . 72

4 Material and Methods $\quad 73$

4.1 Generation of rsEGFP2- $\alpha$-tubulin expressing Drosophila melanogaster 73

4.2 Sample preparation of Drosophila melanogaster . . . . . . . . . . 73

4.3 Western Blot . . . . . . . . . . . . . . . . . . 73

4.4 Western Blot analyis . . . . . . . . . . . . . . . . . . . 74

4.5 Lifespan analysis . . . . . . . . . . . . . . . . . . . . 74

4.6 MAP4-rsEGFP2 Plasmid Construction . . . . . . . . . . . . . . 74

4.7 Generation of MAP4-rsEGFP2 expressing Arabidopsis thaliana. . . . 75

4.8 Arabidopsis thaliana transformation . . . . . . . . . . . . . . . . 75

4.9 Sample preparation . . . . . . . . . . . . . . . . 75

4.10 RESOLFT-microscopy . . . . . . . . . . . . . . . . 75 
4.11 STED-microscopy . . . . . . . . . . . . . . . 76

4.12 FRAP measurements . . . . . . . . . . . . . . . . 76

4.13 Image analysis - Line profiles . . . . . . . . . . . . . . . . . . 77

4.14 Image analysis - Filament detection and counting . . . . . . . . . . 77

4.15 Image manipulations . . . . . . . . . . . . . . . . . . . 77

5 Appendix $\quad 78$

5.1 Determination of average life span . . . . . . . . . . . 78

5.2 Western Blot analysis . . . . . . . . . . . . . . . . . 79

5.3 Sample preparation Arabidopsis thaliana . . . . . . . . . . . . . 80

5.4 References . . . . . . . . . . . . . . . . . . . . . . 81

5.5 List of Figures . . . . . . . . . . . . . . . . . . . . . . . . . . . . . 92

5.6 List of Tables . . . . . . . . . . . . . . . . . . . . . . . 94

5.7 Publications and use of published material . . . . . . . . . . . . 95

5.8 Acknowledgements . . . . . . . . . . . . . . . . . . . 96

5.9 Curriculum vitae . . . . . . . . . . . . . . . . . . . . 98 


\section{Introduction}

Light microscopy enables real-time observation of living specimens. Since the discovery of the cell by R. Hooke in the year 1665, light microscopes have become an indispensable research tool in the field of biology [1]. The continued improvement of light microscopes facilitated unprecedented new insights into cellular substructures and processes; however, many unstained biological samples lack the contrast required to visualize features in regular bright-field microscopy. The development of modern fluorescence microscopy together with new labelling techniques allowed for the specific visualisation of cellular proteins and structures.

In the following sections the fundamentals of fluorescence microscopy are described in detail.

\subsection{Fluorescence microscopy}

\subsubsection{Fluorescence}

Fluorophores absorb light of a defined wavelength region, the so called molecule specific absorption spectrum. Absorption of a photon by the molecule leads to a transition to higher energetic states, which can be grouped into singlet $\left(S_{n}\right)$ and triplet states $\left(\mathrm{T}_{\mathrm{n}}\right)$ (Figure 1.1).

Reversion to the energetic ground state takes place rapidly via emission of a photon [2]. The average time between excitation of the molecule and return to the ground state via fluorescence emission is defined as the fluorescence-lifetime $(\tau)$, which typically falls in the time range of several nanoseconds (ns).

The emitted photon possesses less energy in comparison to the excitation photon due to its rapid decay towards the lowest vibrational level of the $\mathrm{S}_{1}$ state before emission (Figure 1.1). The loss in energy leads to a red shift in the wavelength of the emitted photon, denoted as the Stokes shift.

Aside from spontaneous relaxation via emission of a photon, excited molecules can also be triggered to de-excite immediately. This can be achieved by illumination of the excited molecule with light of a wavelength identical to the emission spectrum of the fluorophore. This light depopulates the excited $S_{1}$ state to the energetic ground state $\mathrm{S}_{0}$ via stimulated emission. A photon with the exact same wavelength, polarization and direction as the stimulating photon is emitted.

The relaxation of excited singlet states $\left(\mathrm{S}_{\mathrm{n}}\right)$ to the ground state $\left(\mathrm{S}_{0}\right)$ can occur via non-radiative processes, a process called Internal Conversion (IC). 


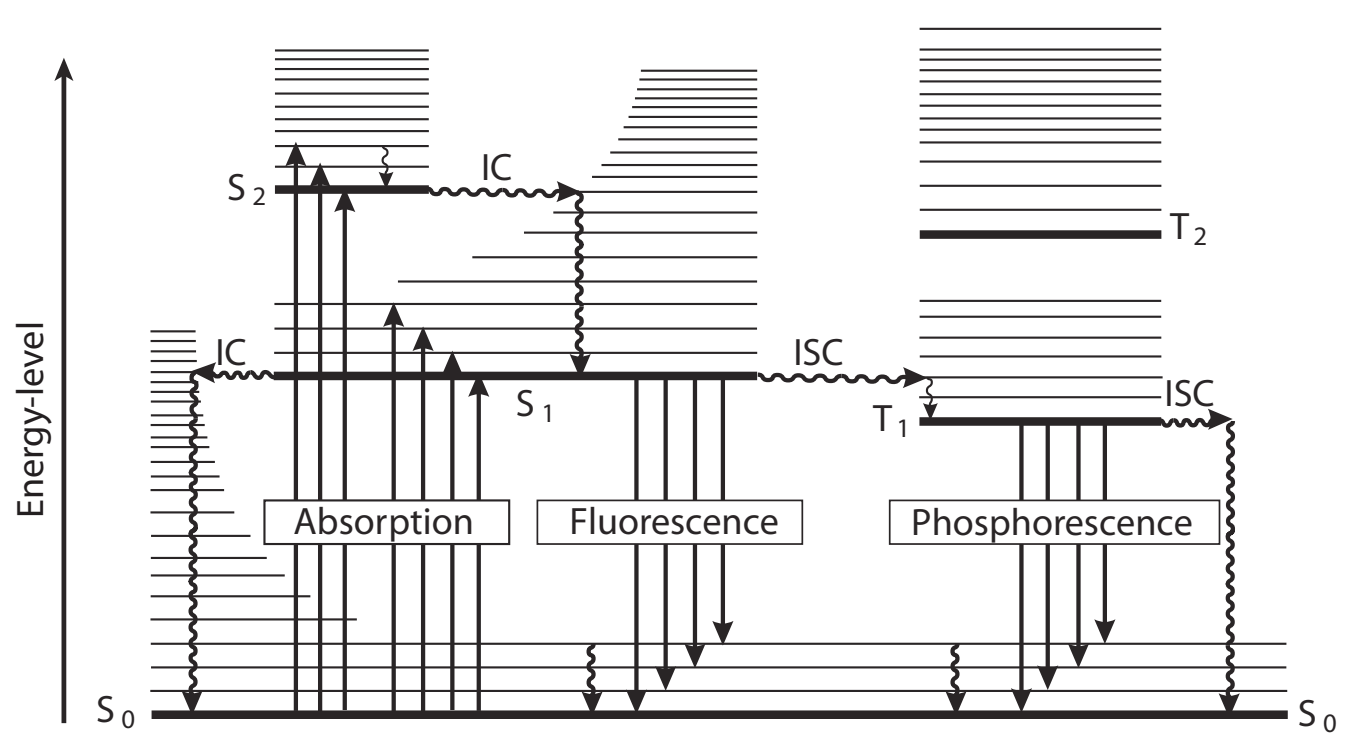

Figure 1.1: Jablonski chart of energetic states of a fluorescent molecule. From the energetic ground state $\mathrm{S}_{0}$, molecules can absorb photons to be transferred into higher energetic states $\left(S_{n}\right)$. Fluorescence occurs from the $S_{1}$ state. ISC leads to triplet state formation $\left(\mathrm{T}_{\mathrm{n}}\right)$. De-excitation out of a triplet state happens via phosphorescence or via ISC. Straight arrows indicate radiative processes, wave arrows indicate non-radiative processes. IC: Internal conversion; ISC: Inter system crossing (Figure modified after [3]).

Here the excitation energy is mostly transferred into vibrational interactions between the molecule and the surrounding solvent molecules.

The probability of a fluorophore to emit a photon instead of de-excitation via a nonradiative pathway is denoted as the molecule specific fluorescence quantum yield $(\Phi)$, which is described as the ratio of emitted photons per number of absorbed photons. The molecule specific photon absorption is given by the extinction coefficient $(\varepsilon)$. The product of the quantum yield and the molecular extinction coefficient is denoted as the brightness $(B)$ of a fluorescent molecule.

\subsubsection{Fluorescence microscopy}

In all fluorescence microscopes, the Stokes shift of the emitted fluorescence photons is used to separate the excitation light from the emitted photons using wavelength selective filters. All modern fluorescence microscopes share the same basic components: a light source for the excitation of the fluorophores, lenses and mirrors to focus and align the light paths, dichroic mirrors for separation of the excitation and emission light and a detector to register the emitted fluorescence emanating from the sample. In a classical wide-field microscope, the excitation light is provided by a high power lamp (e.g. a mercury lamp) and is focused by the objective lens to the 


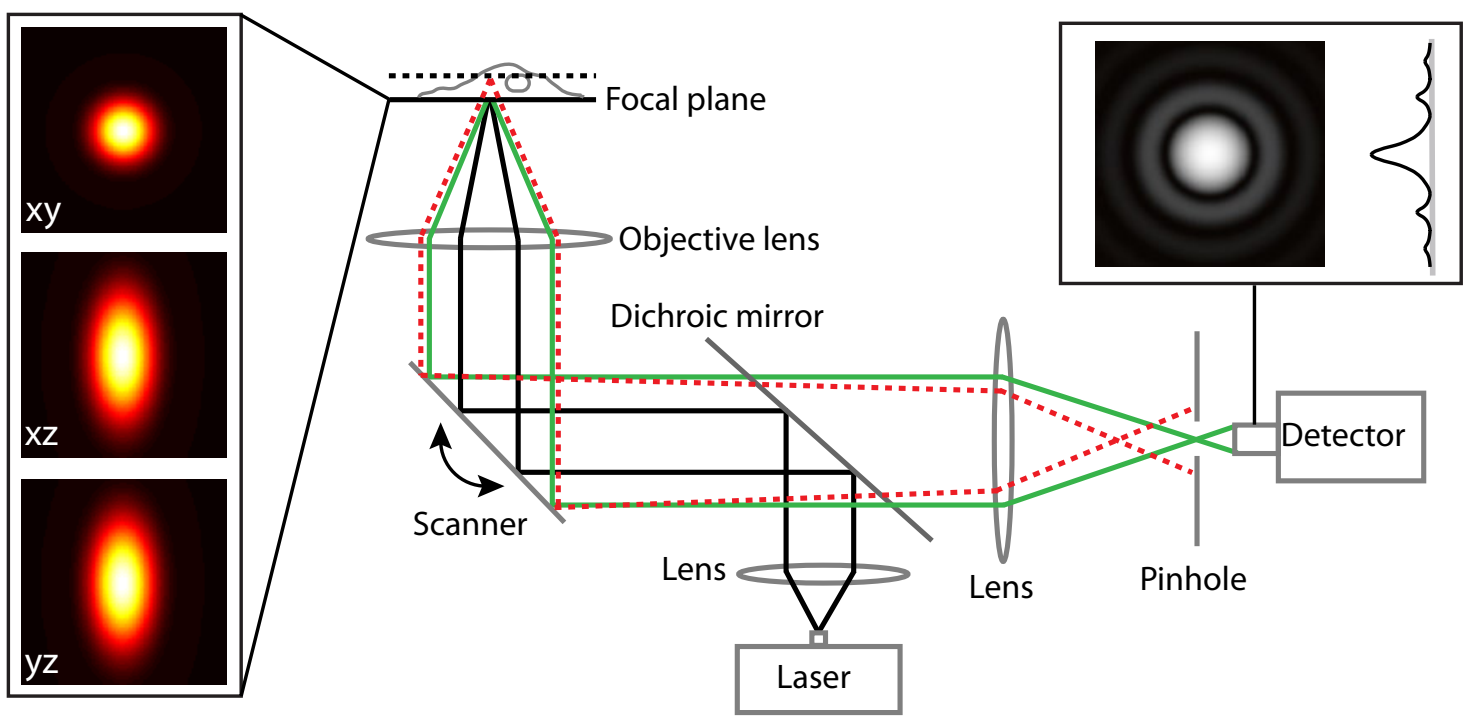

Figure 1.2: Sketch of a scanning confocal microscope. The excitation light which originates from the light source (Laser) is depicted by black lines and is focused by an objective lens into the sample. The emitted fluorescent light from the focal plane is collected by the same objective lens and is illustrated by green lines. The fluorescent light can pass through the dichroic mirror and is focussed onto a detector. Light coming from out-of-focus planes is represented as dotted red lines and is blocked by the pinhole. The scanner moves the excitation light over the sample. The inset on the left side shows simulated excitation point spread functions (PSF) in the lateral (xy) and axial (xz, yz) directions in the focal plane. The inset in the upper right corner depicts the airy pattern at the detector. The central peak flanked by the first minima is denoted as the Airy Disc. The plot on the right side shows the cross section of the intensity distribution of the Airy pattern (Adapted from [4]).

focal plane. The size of the illuminated area depends on the objective lens and is typically in the range of $20 \mu \mathrm{m}-50 \mu \mathrm{m}$ for a $100 \mathrm{x}$ oil objective lens. The detector is typically an electronic camera, which records the emitted fluorescence from the illuminated focal plane. The resolution of the recorded image depends on the objective and the pixel size of the CCD-(Charged Couple Devices) camera detector used. However, the resolution is limited due to the diffraction-limit of light (Section 1.2). A drawback of wide-field excitation is that the excitation light is also absorbed by fluorophores located above and below the focal plane. This will contribute to the image formation, resulting in a background signal. The excitation light can also induce autofluorescence of the sample, which can perturb the image quality further as it is also registered by the detector.

To improve the signal to noise ratio, confocal laser scanning microscopy (CLSM) has been invented. Here the excitation light source is a laser of certain wavelength which is focused by an objective lens to an excitation spot (Figure 1.2). The excita- 
tion spot is scanned over the sample while the emitted fluorescence is detected with a single photon counting module. The number of detected photons per scanning position are counted and used to reconstruct the image digitally pixel by pixel.

A confocal microscope offers optical sectioning of a sample via a pinhole in the detection pathway. The emitted fluorescent light from the focal plane passes through the pinhole in the detection pathway, whereas the light coming from out-of-focus areas are blocked by the pinhole (Figure 1.2). Optical sectioning of a confocal microscope thus allows imaging of large three dimensional samples with improved signal to noise ratio at large imaging depths. The optical resolution of the image in a scanning confocal microscope depends on the excitation spot size and the size of the pinhole used to record the image (Section 1.2).

\subsection{Theoretical resolution of a light-microscope}

Microscopes have undergone substantial instrumental improvements and facilitated better images with more detail and contrast. The improvement was initially focused on increasing the resolution of the instrument, meaning the ability to focus the excitation light to smaller spot sizes. After a certain point, the improvement in optical resolution was no longer limited by the quality of the optical system itself, it was limited by physical laws.

In light microscopes, a point-like emitter is transferred by the optical system to an intensity distribution along the lateral and axial axes, called a point spread function (PSF). The PSF possesses a cylindrical symmetry with maximum intensity at the focal point. The intensity distribution of the PSF is extended along the z-axis (Figure 1.2). The fluorescence emitted by an point like emitter is detected by the microscope as the so called Airy pattern, which features a central peak of maximum intensity which is surrounded by several weaker minima and smaller maxima. The central peak flanked by the first minima is denoted as the Airy Disc (Figure 1.2) [5]. The minimal size of the excitation volume is limited by the so called diffraction-limit which was first described by Ernst Abbe in 1873 [6]. Following Abbe, two point like emitters can not be separated if they are located closer together than $\lambda /(2 \mathrm{NA})$, where $\lambda$ is the wavelength of the emitted light and NA is the numerical aperture of the objective lens.

To separate two point like emitters using an optical system, the Rayleigh criterion has to be fulfilled [7]. For the separation, the distance between the two emitters has to be at least so large that the maximum of the Airy pattern from the first molecule can be separated from the first minimum of the Airy pattern of the second molecule. The optical resolution of a light microscope is described by the minimal distance of 
two points that can be optically separated. To separate two point-like emitters in the lateral plane, the minimal distance $(d)$ between the emitters can be described as:

$$
d=0.61 \times \frac{\lambda}{n \sin \alpha}=0.61 \times \frac{\lambda}{\mathrm{NA}}
$$

Analogous to this, in order to separate two point-like emitters in the axial direction, the minimal axial distance $(z)$ of these two emitters is described by:

$$
z=2 \times \frac{n \times \lambda}{(N A)^{2}}
$$

As can be seen from equations 1.1 and 1.2, the resolution of a light microscope is limited by the wavelength of light $(\lambda)$, the refractive index $(n)$ of the immersion medium and the semi-aperture angle $(\alpha)$ of the objective lens of the microscope. The product of the refractive index $(n)$ and the sine of the semi-aperture angle $(\alpha)$ of the objective lens is denoted as the Numerical Aperture (NA).

The central peak of the Airy pattern of a small point like emitter is often approximated by a Gaussian function. The resolution in the lateral plane is hereby defined as the Full Width at Half Maximum (FWHM) of the fitted function of the central peak of the Airy pattern.

The resolution of a light microscope is fundamentally limited by the diffraction of light. This resolution limit impedes the characterisation of cellular structures featuring a size below this resolution limit by light microscopy. To increase the resolution of a fluorescence microscope, shorter wavelengths and higher NA objective lenses can be used. However, fluorescence microscopy is primarily based on visible light $(400 \mathrm{~nm}-750 \mathrm{~nm})$ and standard objective lenses are limited to a NA of $\sim 1.45$. Following this, the resolution is limited to $\sim 200 \mathrm{~nm}$ along the lateral axis and to $\sim 600 \mathrm{~nm}$ along the axial axis using $488 \mathrm{~nm}$ as the excitation wavelength.

For special microscopic applications, objective lenses with high NA values of 1.7 have been developed. These objectives are used for Total Internal Reflection Fluorescence (TIRF)-microscopy, where the excitation light illuminates through an inclined angle, which leads to an exponentially decaying evanescent wave of excitation light at the coverslip-medium interface (100 nm - $200 \mathrm{~nm}$ along the axial axis) [8]. This causes semi high-resolution imaging along the z-axis, but limits the application of TIRFmicroscopy to structures located directly at the apical membrane of cells, close to the coverslip-cell interface. 


\subsection{Breaking the diffraction-barrier of light microscopy}

The resolution limitation of light microscopy has been overcome by the development of various nanoscopy methods. These methods are based on the utilization of separable states of fluorophores within the diffraction-limited excitation area of a light microscope, which must be distinguishable for the time point of detection [9]. For fluorescent molecules, in the simplest implementation, these states can be a fluorescent and a non-fluorescent state. The nanoscopy methods can be grouped into the so called coordinated-targeted and coordinated-stochastic methods, which will be described in detail in the following Sections.

\subsubsection{Coordinated-stochastic methods}

In the coordinated-stochastic methods, the fluorescence of multiple fluorophores in the excitation volume is separated on the temporal scale. Stochastic methods are mainly based on a wide-field microscope, where the detection is camera based and images a large field of view (Section 1.1.2). In conventional wide-field microscopy, all fluorophores in the sample plane are excited simultaneously. The emitted photons are concurrently detected by a camera, hereby losing the information from which molecule the emitted fluorescence originates. If fluorophores are located closer than $\lambda /(2 \mathrm{NA})$ together, their fluorescence can not be separated and they appear as one. In stochastic localisation microscopy, only a subset of fluorophores are emitting fluorescence in one detection time-span, e.g. one camera-frame. This can be achieved, among others, by using photoactivatable fluorescent proteins which are only activated sparsely to ensure that the fluorescence emission of a single molecule is not overlapping with the emission of a second one on a single recorded camera frame (Figure $1.3 \mathrm{a}$ ). If this is achieved, the position from which the fluorescence came from, can be determined with sub-diffraction precision. In the post-processing of the recorded data, the diffraction blurred spot on the camera frame is fitted and the center-position (center of mass) is calculated (Figure $1.3 \mathrm{~b}$ ) [10].

The fluorophores have to be able to emit a sufficient number of photons before they are bleached, because the localization precision of a molecule scales approximately with the inverse square root of the number of photons emitted by the fluorophore. Localisation itself does not provide high-resolution imaging intrinsically. Resolution is described by separating two emitters that are located closely together. The position of single fluorophores can be calculated with virtually unlimited precision, although, if they are located closer together than $\lambda /(2 \mathrm{NA})$, their fluorescence can 
a
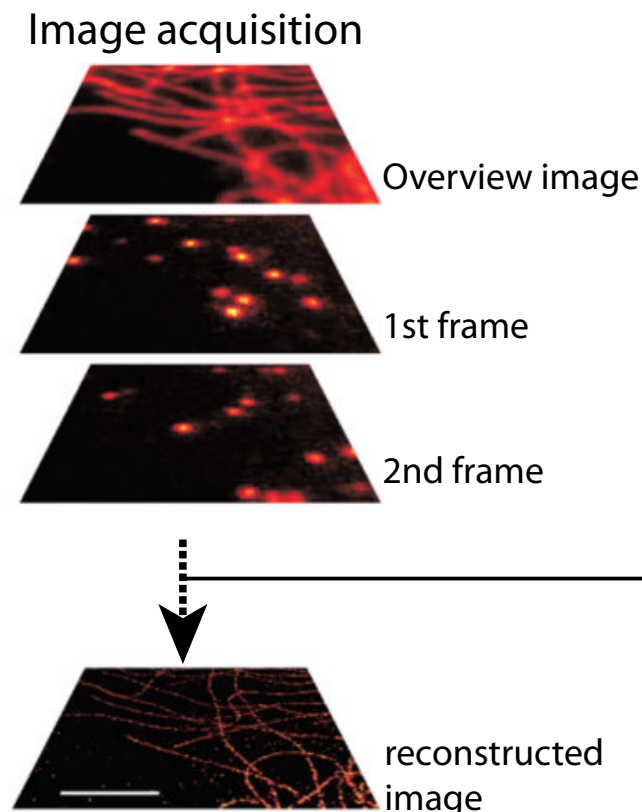

reconstructed

image b Data processing

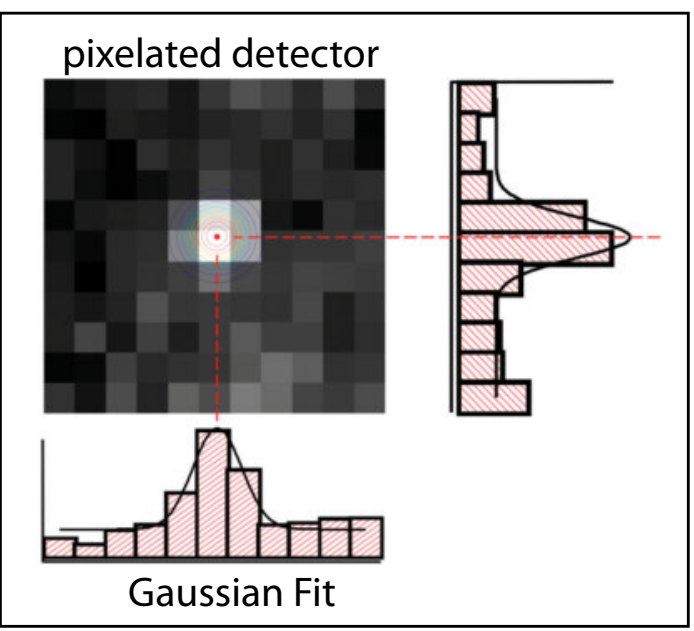

Figure 1.3: Principle of coordinated-stochastic imaging methods. a) Upper image: Overview image of labelled structure. From this sample, multiple images are recorded, whereby only a subset of fluorophores are excited/activated simultaneously which are spaced further apart than the resolution limit. b) Pixelated PSF of a single fluorophore detected by the CCD-camera and modelled by a three-dimensional Gaussion function. For each fitted Gaussion profile of a fluorophore, the centroid position is calculated. By combining all calculated centroid positions of all fluorophores, a high-resolution image of the labelled structure is reconstructed. Scale bar: $2 \mu \mathrm{m}$ (Image modified after a) [11] and b) [12]).

not be separated due to the diffraction-barrier of light. Only by using separable states within a diffraction-limited distance, these fluorophores become separable.

After detection of the fluorescence, the molecules are bleached or off-switched and another subset is activated (or excited). By performing thousands of imaging and localization steps, a high-resolution image displaying the calculated positions of the individual fluorophores is computed (Figure 1.3).

Several different experimental designs have been introduced which are all based on this general stochastic design of imaging. Beside (fluorescence)-Photoactivated Localization Microscopy ((f)-PALM) [13], [14], STochastic Optical Reconstruction Microscopy (STORM) was independently reported in 2006 [15]. These methods differ in their utilised fluorophores, f-PALM relies on photo-activatable fluorescent proteins, whereas STORM is based on single or pairs of organic dyes. The lateral resolution of STORM and PALM is typically between $20 \mathrm{~nm}-30 \mathrm{~nm}$ [13], [14].

The axial resolution of stochastic targeted methods can be improved using optical astigmatism to calculate the localization of fluorophores along the z-axis in the 
sample, resulting in axial resolutions of $50 \mathrm{~nm}-60 \mathrm{~nm}$ [16]. A main limitation of the coordinated-stochastic methods is the time consuming recording of hundreds to thousands of single frames, which are needed to reconstruct a single high resolution image. These long acquisition times make these methods largely unsuitable for live cell imaging.

\subsubsection{Coordinated-targeted methods}
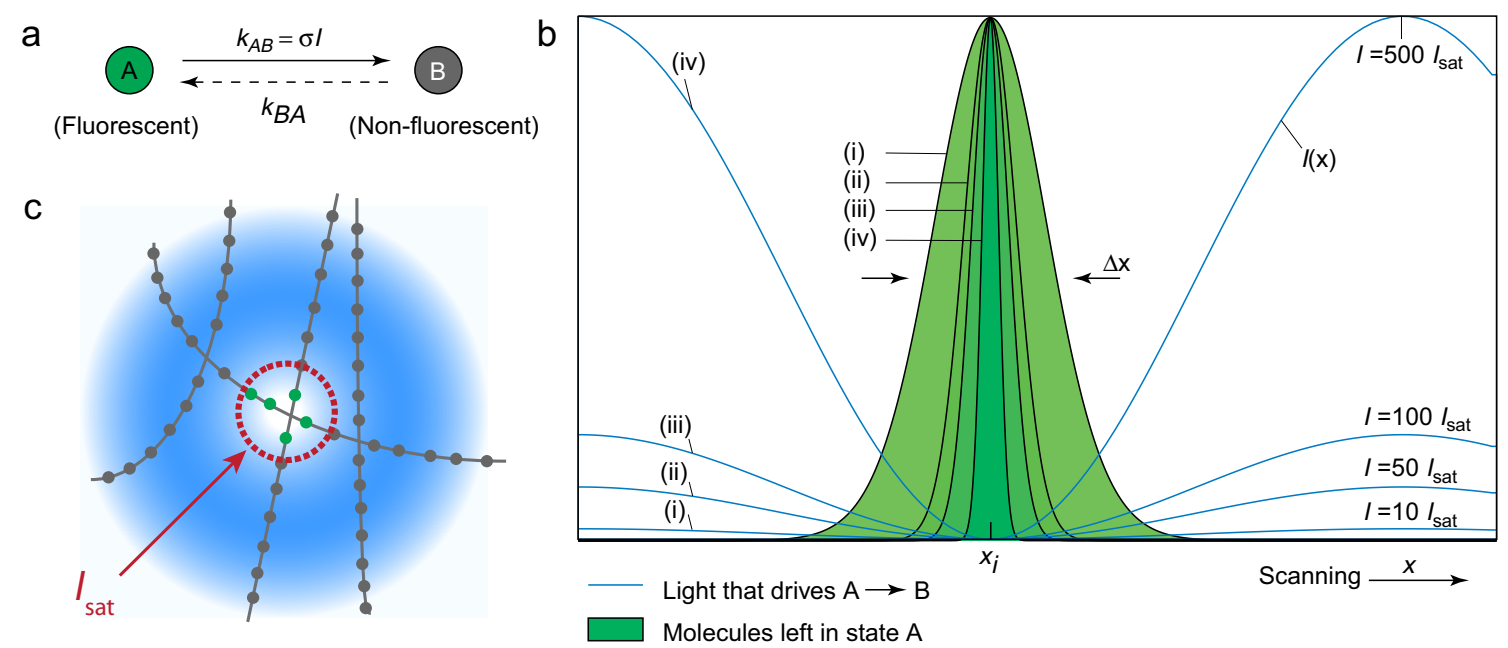

Figure 1.4: Principle of RESOLFT-microscopy. a) Two state model of fluorescent molecules featuring a light driven transition. The rate constant $k_{A B}=\sigma \times I$ is dependent on the light intensity $I$ and the absorption cross section $\sigma$. b) Onedimensional exemplified confinement of molecules residing in state A by applying different multiples of $I_{\text {sat }}$ (i,ii,iii,iv) and with zero intensity at position $x_{i}$. c) Graphical two dimensional example illustrating the transition of flourescent molecules (dots) located at an illustrated target structure to a flourescent off-state (state B - gray color) by applying light with intensity $I>>I_{\text {sat }}$. Depending on the light intensity of the off-switching light pattern, the size of the area where molecules stay in state A can be modified (Modified after [17]).

In the coordinated-targeted methods, a light pattern is used to precisely transfer a subset of molecules within the diffraction-limited excitation spot into a second state, which is discriminable from the first state. In case of fluorescent molecules, the easiest two discriminable states are a fluorescent (e.g. state A) and a non-fluorescent state (state B) (Figure 1.4 a). The light pattern features at least one position of zero intensity to confine a small, sub-diffraction sized area in which molecules will stay in their fluorescent on-state (state A) [17].

The light driven transition into a state can be described with a rate constant $k_{A B}=$ $\sigma \times I$, with $\sigma$ describing the absorption cross section and $I$ the intensity of the light which drives the transition (Figure 1.4 a). The possibility of a molecule to be in 
state $\mathrm{A}$ or $\mathrm{B}$ is thereby depending on the rate $k_{A B}$, i.e. on the light intensity $I$ which drives the transition. At the saturation intensity $I_{\text {sat }}$, the number of molecules in state $\mathrm{A}$ and $\mathrm{B}$ are equal. At intensities $I>>I_{\text {sat }}$ the molecules are transferred into state B. The possibility to be in state A or B for a molecule is dependent on the light intensity $I_{\text {sat }}$ driving the transition, which is highly non-linear (Figure $1.4 \mathrm{~b}$ ) [17].

This non-linear light driven transition into a second state is used in coordinatedtargeted microscopy to confine a sub-diffraction sized spot of molecules residing in state A, i.e. a fluorescent state, which can be read-out. Practically, this is achieved by illuminating the molecules in the focus with a second light pattern which drives the transition to the second state (state B).

The easiest implementation is a doughnut-shaped laser beam, which features a center position of zero intensity $(I=0)$ (Figure $1.4 \mathrm{c}$ ). Apart from the center position, this beam drives the transition of molecules to a fluorescent off-state at positions where the light intensity is $I>>I_{s a t}$. Given this, the diffraction-limited spot of fluorescent molecules can be narrowed to a sub diffraction sized spot of addressable molecules (Figure $1.4 \mathrm{c}$ ).

The state-transition must be saturated to realize a good separation between the states, hereby enabling the separation at the time point of detection. The size of the effective excitation area can be tuned by the light intensity $(I)$ of the offswitching beam (Figure 1.4 b). Its spatial size can be described using the formula:

$$
\Delta d=\frac{\lambda}{\pi n \sqrt{I / I_{\text {sat }}}}
$$

Subsequently, the resolution formula from Abbe (1.1) can be modified to describe the size of the effective excitation spot size of a microscope following the RESOLFTprinciple:

$$
\Delta d=\frac{\lambda}{2 n \sin \alpha \sqrt{1+I / I_{s a t}}}
$$

The achievable resolution is in principle unlimited and only depends on the light intensities which drive the transitions $\left(I\right.$ and $\left.I_{\text {sat }}\right)$. The practical limitations consist of the availability of powerful light sources and the amount of light a sample can tolerate without damage. By using a state transition featuring a low $I_{\text {sat }}$, the intensity needed to achieve high-resolution can be decreased.

In the coordinated-targeted methods, the positions of emitting and non-emitting molecules are defined spatially by illuminating the molecules with a light pattern which drives the respective transition locally. The effective PSF (i.e. the combina- 
tion of excitation and depletion PSFs) of the microscope is much smaller than the corresponding diffraction-limited PSFs.

The underlying principle can be extended to all discernible pairs of states that feature a light driven transition which can be saturated. Thus, this general principle has been termed Reversible Saturable Optical Fluorescence Transition (RESOLFT)microscopy [18], [19], [20].

\subsubsection{RESOLFT-type microscopy techniques}

Stimulated Emission Depletion (STED)-microscopy was the first experimental demonstration of the RESOLFT-principle utilizing a state separation within a diffraction limited excitation volume, to overcome the diffraction limit [21], [22]. Here, the pair of separable states of fluorescent molecules are the non-fluorescent ground state $\mathrm{S}_{0}$ and the excited fluorescent state $\mathrm{S}_{1}$. Next to an excitation pulse, which transfers molecules to the excited $\mathrm{S}_{1}$ state, a second superimposed offswitching laser is used to de-excite molecules in the periphery of the excitation area via stimulated emission (Section 1.1). The photons which are emitted via the stimulated emission possess the same wavelength as the off-switching beam, thus these photons can be discarded by using wavelength selective filters. Following this, only the fluorescence of the molecules remaining in the excited $\mathrm{S}_{1}$ state, located at the very center of the excitation area, are detected. The de-excitation process must outperform the spontaneous fluorescence emission, which features a lifetime of $\sim 1-4$ ns for typical fluorophores [23]. Therefore, the light intensities used in STEDmicroscopy are relatively high (several tens to a hundred $M W / \mathrm{cm}^{2}$ ) to achieve a saturated de-excitation before the spontaneous emission occurs [17].

In Ground State Depletion (GSD)-microscopy, fluorophores are separated by transferring a subset of excited molecules from their $\mathrm{S}_{1}$ state into a dark long-lived triplet state $\left(\mathrm{T}_{1}\right)$, for example by using a doughnut shaped beam [24]. Within the $\mathrm{T}_{1}$ state, the molecules are not addressable by the excitation beam to emit fluorescence at the time point of detection. A drawback is the fact that fluorophores situated in the triplet state are prone to bleaching [25].

The high light intensities used in STED-microscopy may be unfavourable for imaging of living cells. By utilizing separable stable states which feature a long lifetime, the transition between the states can be driven with very low light levels. Reversibly Switchable Fluorescent Proteins (RSFPs) can be targeted switched between longlived fluorescent and non-fluorescent states, which are stable for minutes or hours using visible light [20]. The switching of the fluorescent state is based on a light driven conformational change of the chromophore of these RSFPs [26]. The utilization of this photo-switching in RSFP-based RESOLFT-microscopy is described 
in Section 1.4.4. In this work RESOLFT-microscopy is performed using RSFPs; therefore, RESOLFT-microscopy is used synonymously for RSFP-based RESOLFTmicroscopy in the following.

The nonlinearity of photo-switchable fluorescent proteins is used in saturated structured illumination microscopy (SSIM) to confine molecules in a sub-diffraction sized area in the ground state $S_{0}$. This is realized by illuminating the sample with an excitation light pattern featuring intensity minima and maxima. The nonlinearity of excitation leads to narrow regions where the molecules remain in the ground state $\mathrm{S}_{0}$ flanked by areas of saturated excitation. The excitation grid pattern is rotated several times and a microscopic image is reconstructed from of the recorded negative images [27]. In combination with TIRF-illumination, the axial resolution of SSIM can be restricted to imaging of the apical cell membrane of single cells with high frame rate and high-resolution in the axial plane [28].

\subsection{Reversibly switchable fluorescent proteins}

\subsubsection{Photophysical properties of RSFPs}

The first characterized fluorescent protein was the Green Fluorescent Protein (GFP) found in the jellyfish Aequorea victoria denoted as avGFP [29]. The cloning of the GFP-gene enabled the heterologous expression in many biological organisms and it is presently widely used in fluorescence microscopy to image the protein of interest in living cells as a GFP-fusion construct [30].

GFP is a monomeric protein with a molecular size of about $27 \mathrm{kDa}$. All GFP-type fluorescent proteins consist of $\sim 240$ amino acids which form a barrel consisting of eleven co-axial oriented $\beta$-sheets. The barrel encases an $\alpha$-helix of $\sim 14$ amino acids containing the chromophore, thereby shielding it from the surrounding solvent molecules [31]. The chromophore is formed auto-catalytically from three amino acids of the amino acid chain in the presence of molecular oxygen and without any cofactors [32].

Upon excitation of the chromophore, the barrel restricts movement of the excited chromophore via several hydrogen bonds and via steric hindrance, hereby increasing the radiative de-excitation mechanisms. The importance of this stabilisation is accentuated by the fact that a free moving synthetic GFP chromophore in an aqueous solution shows dramatically reduced brightness. This is most likely due to a reduction of excitation energy via non-radiative processes e.g. vibration of the chromophore and interactions with solvent molecules [33]. 
In many fluorescent proteins, the imidazol ring and the p-hydroxyphenyl ring of the chromophore can be protonated. Protonation of the hydroxyphenyl group of the GFP-like proteins often lead to a blueshift of $\sim 100 \mathrm{~nm}$ in the absorption peak of the chromophore [32].

In avGFP, the chromophore can be found protonated as well as unprotonated, which is visible in the absorption spectra as two peaks (at $\sim 390 \mathrm{~nm}$ and $\sim 475 \mathrm{~nm}$ ) [32]. Excitation at any absorption peak leads to emission of green fluorescence at $\sim 505 \mathrm{~nm}$. It was shown that the excitation of the neutral chromophore leads to a proton transfer from the tyrosyl of the chromophore to glutamic acid at position 222 (Glu222) within picoseconds. This process is denoted Excited State Proton Transfer (ESPT). The intermediate product emits at $\sim 505 \mathrm{~nm}[34]$. A single point mutation of eGFP (enhanced GFP) shifts the absorption spectra almost exclusively to a single peak at $\sim 490 \mathrm{~nm}$, demonstrating that the chromophore is existing mostly in the anionic state. The $\mathrm{S} 65 \mathrm{~T}$ mutation rearranges the hydrogen bond pattern of Glu222, suppressing its negative charge. By this, an interaction is suppressed and the chromophore stays almost exclusively deprotonated [35].

In the year 2000, Lukyanov et al. reported on the fluorescent protein asFP595 which was found in Anemonia sulcata [36]. This tetrameric fluorescent protein showed a switchable fluorescent behaviour. Excitation with light of $\sim 550 \mathrm{~nm}$ led to the emission of fluorescence at a peak of $\sim 595 \mathrm{~nm}$ whereas light of $\sim 450 \mathrm{~nm}$ led to a reversible reduction of fluorescence.

The discovery of this reversible switchable fluorescence of a fluorescent protein opened up the field to a new class of optical highlighters of fluorescent proteins. A protein which possess such a reversible switchable behaviour is termed Reversible Switchable Fluorescent Protein (RSFP). However, the tetrameric nature of asFP595 makes it unfavourable as a genetically encoded label for protein-tagging in living systems. For this purpose, monomeric proteins are highly desired.

In 2004, Ando et al. described the first monomeric RSFP which was named Dronpa (Figure 1.5) [37]. It was found in monomerisation studies with fluorescent proteins of Pectiniidae. This protein features a reversible switchable fluorescent characteristic similar to asFP595, but in the green spectral region and was used to track the diffusion of proteins inside living cells. Interestingly, asFP595 and Dronpa show an inverse switching behaviour. In asFP595, the excitation-wavelength drives the transition to the fluorescent on-state whereas in Dronpa the excitation leads to the off-switching to its non-fluorescent off-state. Depending on the switching behaviour upon excitation, RSFPs are grouped into positive (e.g. asFP595) or negative-switching (e.g. Dronpa). 


\subsubsection{Switching mechanism of RSFPs}
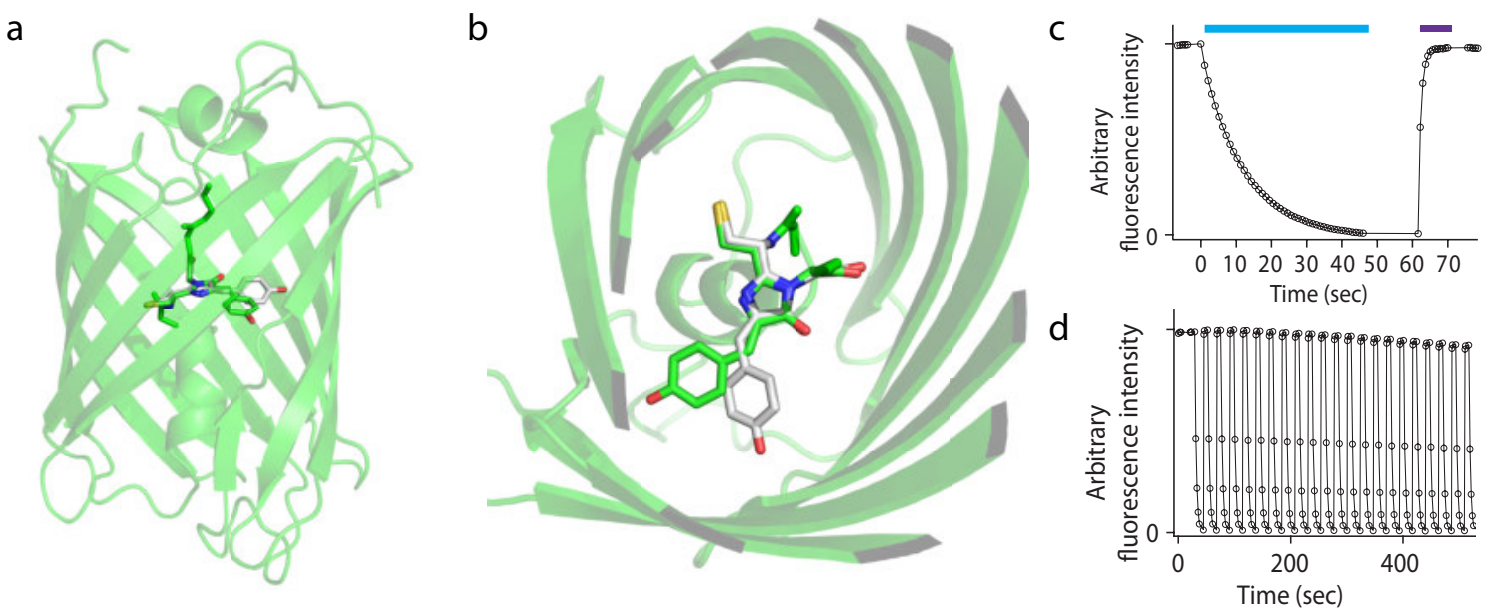

Figure 1.5: Crystal structure and photo-switching of Dronpa. a) Cartoon ribbon plot of Dronpa crystal structure, the chromophore is depicted in stick model in the cis-(green) and trans-(gray) conformation. b) Close-up of the chromophore in the cis-(green) and trans-(gray) conformation, surrounding amino acids are not shown. c) Switching curve of Dronpa recorded in fixed HeLa cells, illumination with light of $490 \mathrm{~nm}$ (blue bar) leads to reduction in fluorescence, illumination with light of $400 \mathrm{~nm}$ (violet bar) leads to the on-switching and recovery of fluorescence. d) Multiple switching curves at the same position recorded consecutively, showing switching fatigue (i.e. reduction of fluorescence after multiple switching cycles). Light intensities used in c): $490 \mathrm{~nm}\left(0.4 \mathrm{~W} / \mathrm{cm}^{2}\right)$ and $400 \mathrm{~nm}\left(0.14 \mathrm{~W} / \mathrm{cm}^{2}\right)$ and in d) $490 \mathrm{~nm}\left(1.3 \mathrm{~W} / \mathrm{cm}^{2}\right)$ and $400 \mathrm{~nm}\left(0.47 \mathrm{~W} / \mathrm{cm}^{2}\right)$. Dronpa crystal structures used from PDB: 2POX (trans) and 2IOV (cis) (c) and d) are modified after [37]).

Using x-ray crystal-structures of the RSFPs asFP595 and Dronpa in their fluorescent on- and off-states, the molecular mechanism for the switching of these proteins was revealed [26], [38]. During off-switching, the chromophore is changing its conformation from a cis- to a trans-isomerization (Figure 1.5). Albeit, there are also fluorescent proteins which are fluorescent in their trans-state and are non-flourescent in their cis-state [39], [40].

Upon off-switching in Dronpa, the chromophore imidazolinone ring stays almost in place, whereas the p-hydroxyphenyl ring moves by $\sim 5.8 \AA$ via rotation around the methine bridge. This translocation of the p-hydroxyphenyl ring needs space inside the $\beta$-barrel, which is accomplished by the translocation of chromophore surrounding amino acids [38]. 
a

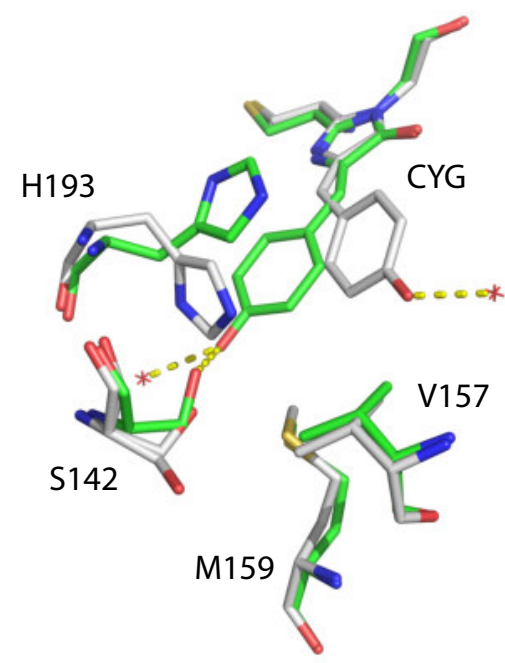

b

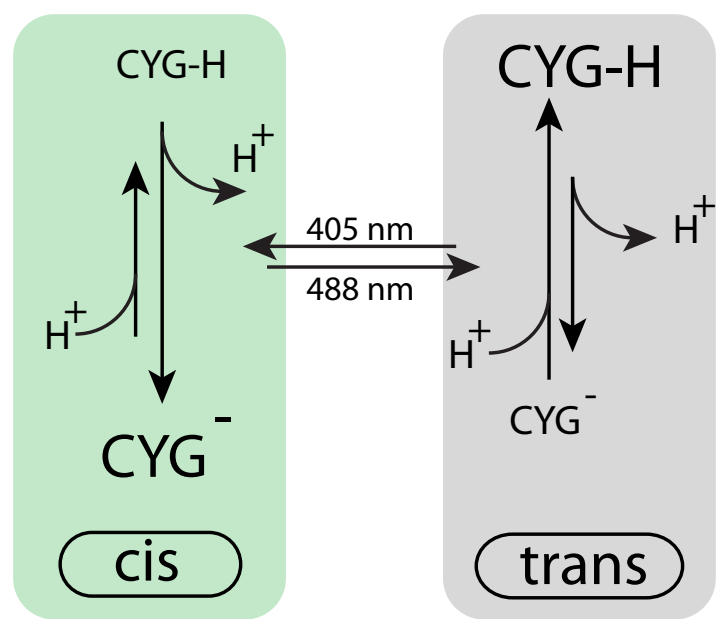

Figure 1.6: Switching mechanisms of the RSFP Dronpa. a) Stick model of the orientation from the Dronpa chromophore (CYG) together with selected surrounding amino acids in the cis- (green) and trans- (grey) state. Water molecules are displayed as red stars. Hydrogen bonds are highlighted with dotted yellow lines. b) Reversibly switching model of Dronpa between the cis- and trans-state and with different protonaton states. Dronpa crystal structures used from PDB: 2POX (trans) and 2IOV (cis) (Figure modified after [38]).

The chromophore is connected via several interactions with amino acids of the $\beta$ barrel. These interactions include hydrogen bonds to other residues, water-mediated hydrogen bonds and van der Waals interactions. Upon off-switching, these interactions are reduced and the chromophore is slightly tilted. The reduction in planarity of the chromophore together with the looser attachment by the $\beta$-barrel leads to the strong reduction of fluorescence of the trans-chromophore [26]. Further, the loose attachment promotes non-radiative de-excitation mechanism after excitation [38]. The different chromophore conformations are thermally stable for seconds to hours, depending of the respective RSFP.

The conformational change of the chromophore is often coupled with a direct change in the protonational state [38]. The negative switching RSFP Dronpa features an almost completely deprotonated chromophore in its cis-state leading to a strong absorbance peak at $\sim 490 \mathrm{~nm}$, which can be adressed by light to excite fluorescence and will cause the off-switching to the trans-state. Upon switching in the non-fluorescent trans-state, the chromophore's hydroxyphenyl group becomes protonated which result in a strong blue-shift in the absorbance spectrum, leading to a peak at 390 $\mathrm{nm}$ [38]. By addressing this UV-absorbance peak, the protein can be switched back to the cis-state again. Due to changes in the electrostatic surface potential of the surrounding amino acids of the chromophore in the different isomerization, the pro- 
tonation state of the chromophore is changing after the cis/trans isomerization [38]. The different states are addressable separately, thus enabling a targeted excitation of different chromophore conformations and facilitate a triggered switching between two different states. In RSFP-based RESOLFT-microscopy, this targeted switching is used to confine a subset of molecules into a sub-diffraction sized area in their fluorescent on-state, while the molecules in the periphery of the excitation volume are switched off.

In positive switching RSFPs, the on-state is partially protonated, hence enabling the targeted off-switching with shorter wavelength compared to the excitation wavelength [41].

So far only one exception from the cis/trans switching-mechanism has been found in the RSFP Dreiklang. Here the switching is decoupled from the fluorescence excitation. The switching is based on a hydration reaction of the imidazolinone ring of the chromophore. In the on-state, Dreiklang exhibits two absorbance peaks, one at $405 \mathrm{~nm}$ and the other at $511 \mathrm{~nm}$. Illumination with light of $405 \mathrm{~nm}$ leads to the addition of water to the imidazolinone ring and thereby to a shortening of the conjugated electron $\pi$-system, resulting in a new absorption peak at $\sim 350 \mathrm{~nm}$, where the absorption at $\sim 510 \mathrm{~nm}$ decreases strongly. This blue shift in the absorbance spectrum, leads to the almost complete absence of fluorescence upon excitation at $488 \mathrm{~nm}$. At illumination with light of $\sim 350 \mathrm{~nm}$, the imidazolinone ring gets dehydrated and the protein is converted back to the fluorescent on-state [42], [43].

\subsubsection{RSFPs used for RESOLFT-imaging}

In recent years several improved or new RSFP variants were reported. These proteins are the product of semi-rational mutagenesis based on x-ray crystal structures of template fluorescent proteins. Here, single or multiple amino acids of the template proteins were changed to modify the photophysical properties of the protein for their application in RESOLFT-microscopy. In a point scanning RESOLFT-microscope, the acquisition time for an image is limited by the switching speed of the RSFP used. Further, the number of switching cycles before bleaching of a RSFP is critical to record multiple frames.

A single amino acid exchange in Dronpa at position 159 (exchange of Methionin by Threonin) improved the off-switching speed by up to a factor of $\sim 1000$ times [44]. The M159T exchange leads to lowering of sterical hinderance of the isomerisation of the chromophore by generating space inside the protein barrel. A drawback is the looser stabilisation of the chromophore in the cis-conformation, leading to a reduction of the quantum yield to 0.23 [44]. 


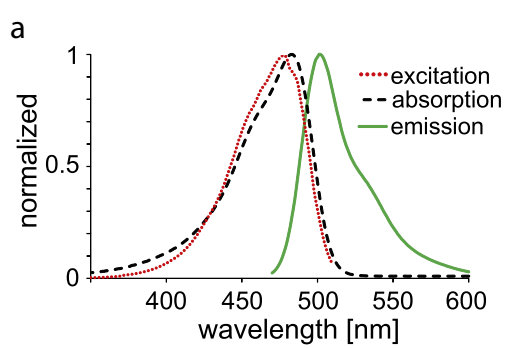

d

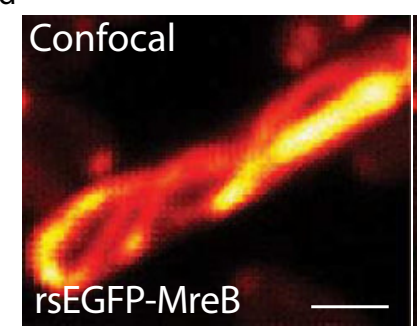

b

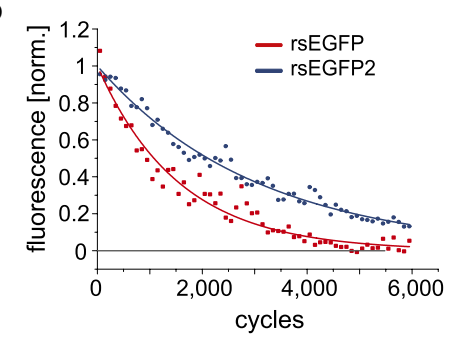

C

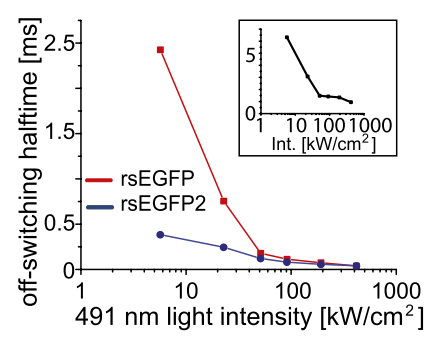

e
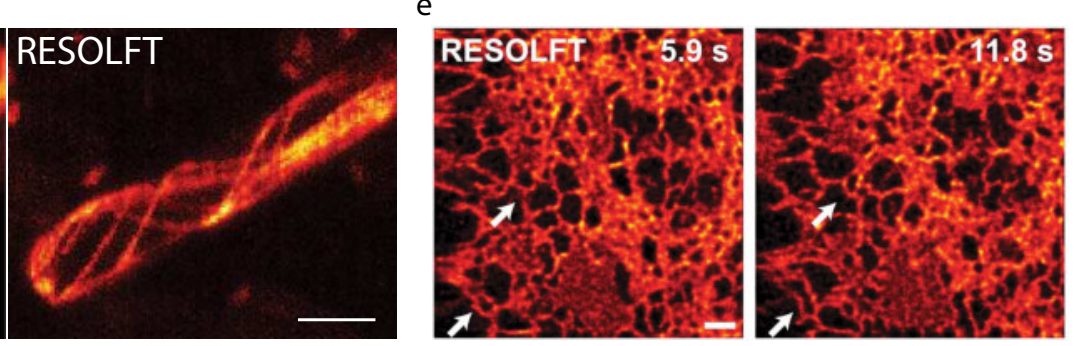

Figure 1.7: Application of rsEGFP and rsEGFP2 in RESOLFTmicroscopy. a) Absorbance, excitation and emission spectra of rsEGFP2 in its equilibrium state at $\mathrm{pH}$ 7.5. The chromophore fascilitate an excitation peak at 478 $\mathrm{nm}$ (red dotted line). The emission of the cis-deprotonated chromophore features a maximum at $\sim 510 \mathrm{~nm}$ (green line). b) Comparision of switching fatigue between rsEGFP and rsEGFP2. c) Comparision of switching speed at different light levels between rsEGFP and rsEGFP2. d) Confocal and RESOLFT-image of rsEGFPMreB fusion protein in living E. coli. e) RESOLFT-microscopy of KDEL-rsEGFP2 in living HeLa-cells, showing the dynamics of the ER. Scale bars: $1 \mu \mathrm{m}(\mathrm{a}), \mathrm{b}), \mathrm{c})$ and e) adapted and modified after [45], d) adapted and modified after [46]).

Later, additional mutations in Dronpa resulted in a reversed switching mechanism which turned Dronpa into the positive switching RSFP Padron [47].

In 2011, Grotjohann et al. [46] reported on rsEGFP, a reversibly switchable version of mEGFP (monomeric enhanced GFP) and demonstrated its application in RESOLFT-microscopy of biological samples as well as for data storage. rsEGFP is a negative switching protein; excitation with light of $488 \mathrm{~nm}$ excites the fluorescence as well as drives the transition to the fluorescent off-state. Upon irradiation with light of $405 \mathrm{~nm}$, the proteins are switched back to the fluorescent cis-conformation. rsEGFP has low switching fatigue (bleaching due to switching), after 1000 switching cycles it is bleached to $\sim 50 \%$ of the initial fluorescence. Further rsEGFP features reasonable brightness and switching speed while still possessing good performance as a genetically encoded tag, being a true monomer.

Using rsEGFP, the application of RESOLFT-microscopy was demonstrated for the first time in living cells. Next to images of MreB-filaments of E.coli and cytoskeleton filaments of mammalian cells, the usability was proven by imaging F-actin fibers in cultivated neurons. Here a resolution of $\sim 70 \mathrm{~nm}$ in E.coli and a resolution of 
$\sim 40 \mathrm{~nm}$ at cytoskeleton filaments of mammalian cells was demonstrated. This implies a resolution improvement by a factor of 4 - 5 times compared to the diffractionlimited confocal resolution. A drawback is the fact that the off-switching time of rsEGFP is in the time range of several milliseconds (ms). While this is a improvement compared to Dronpa, the recording times of single RESOLFT-images were in the time scale of several tens of minutes, complicating the imaging of dynamic processes.

In 2012, Grotjohann et al. reported on the development of rsEGFP2, which possesses a 25 - 250 times faster switching speed at low light levels and improved switching fatigue compared to rsEGFP (Figure 1.7). These properties enabled highresolution RESOLFT-imaging with recording times of seconds in living cells, and thus recording of fast dynamic cellular processes of different structures like Vimentin, Endoplasmic reticulum and Peroxisomes [45].

Based on the mutagenesis on mCherry, the color pallete of RSFPs was extended to monomeric fluorescent proteins in the red-spectral region, resulting in the positive switching rsCherry and the negative switching rsCherryRev [48]. Later rsTagRFP was reported and successfully applied in photochromic Förster resonance energy transfer [49]. However, so far it was not used for RESOLFT-imaging.

The time consuming point-wise scanning of a sample was overcome by Chmyrov et al. in 2013 by parallizing the off-switching step of negative switching RSFPs. Here standing light waves were used to generate an off-switching pattern in a widefield RESOLFT-microscope, featuring more than 100000 doughnuts. The strong parallelization enabled the utilization of a slow switching RSFP variant, termed rsEGFP(N205S) and resulted in RESOLFT images of large fields of view $(\sim 100 \mu \mathrm{m}$ $\mathrm{x} 100 \mu \mathrm{m})$ in less than 3 seconds [50].

In the following years new RSFPs for application in RESOLFT-microscopy were reported. In 2016 Wang et al. reported on an additional negative switching RSFP GMARS-Q for application in parallelized RESOLFT-microscopy. GMars-Q (Greenform Mars) is a monomeric RSFP based on mMaple3, featuring a low residual off-state fluorescence of only $1 \%$ and a strong photo-bleaching resistance. GMars-Q enabled longtime RESOLFT imaging of 50 frames [51].

Further, the application of RESOLFT-microscopy was extended to dual-color live cell imaging by using two spectral separable proteins or by using fluorescent state kinetics to seperate two spectrally similar RSFPs [52] [53].

Next to this, the folding of the rsEGFPs was improved, leading to rsFolder [54] and rsGreen [55]. Both proteins fascilitated RESOLFT imaging with off-switching times comparable to rsEGFP2. In 2015 a modified version of Padron was reported, 
Kohinoor demonstrated RESOLFT imaging with the so far lowest reported light intensities used for RESOLFT-imaging [56]. In 2016 Zhang et al., reported on Skylan-NS (sky lantern for non-linear structured illumination), a RSFP designed for application in non-linear structured illumination microscopy (NL-SIM). It provides slow off-switching speed and very low switching background [57].

All these demonstrations were based on single cells over-expresssing a RSFP-fusion protein, which can induce artefacts in the target structure or influence the fitness of the cell (reviewed by [58]). Endogenous tagging via CRISPR/Cas9 demonstrated that RESOLFT imaging can be performed with RSFP-fusion proteins expressed at physiological levels in single mammalian cells [59].

\subsubsection{Point scanning RESOLFT-microscopy using RSFPs}

In a point scanning RESOLT-microscope, RESOLFT imaging with RSFPs is based on performing multiple illumination steps at each scanning position to trigger the on-, or off-switching of RSFPs in the focal spot. In the following, the standard RESOLFT imaging scheme utilizing the negative switching rsEGFP2 is exemplified [45]. To record a RESOLFT image using a point scanning RESOLFT-microscope, three illumination steps are performed at each scanning position. First, illumination with light of $405 \mathrm{~nm}\left(2 \mathrm{~kW} / \mathrm{cm}^{2}\right)$ for $40 \mu$ s to activate all rsEGFP2 molecules within the excitation spot. Next, deactivation of molecules in the periphery of the excitation volume via a doughnut-shaped off-switching beam featuring a central position of zero intensity, using light of $488 \mathrm{~nm}\left(10 \mathrm{~kW} / \mathrm{cm}^{2}\right)$ for $300 \mu \mathrm{s}$. Last, readout of molecules that remained in their fluorescent-on state which were located in the center of the doughnut-shaped beam using light of $488 \mathrm{~nm}\left(38 \mathrm{~kW} / \mathrm{cm}^{2}\right)$ for $30 \mu \mathrm{s}$.

Here, the recorded fluorescence originates from a sub-diffraction sized area, which is confined in its size by the light intensity of the doughnut-shaped off-switching beam (Figure 1.4). The above mentioned imaging parameters have to be adapted to the respective sample depending on label density and movement of the target structure while imaging. To achieve a high-resolution RESOLFT image with good signal to noise ratio, each switching step has to be executed with best efficiency.

Using a negative switching RSFP for imaging, the signal per scanning position is limited by the off-switching of the utilized RSFPs while readout. Thus, in sparsely labelled samples, multiple iterations of the three illuminations steps have to be performed at each scanning position and the signal has to be accumulated. 
1. On-switching

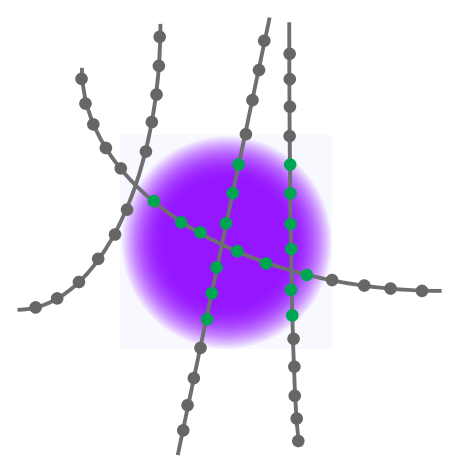

2. Off-switching

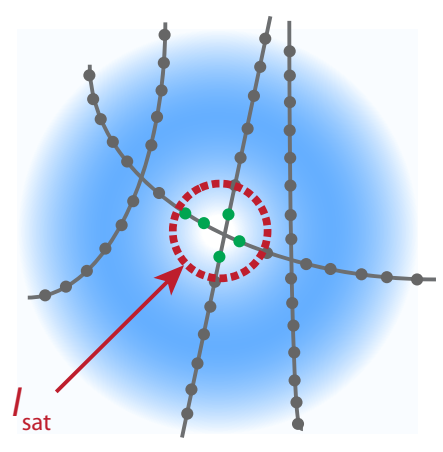

3. Readout

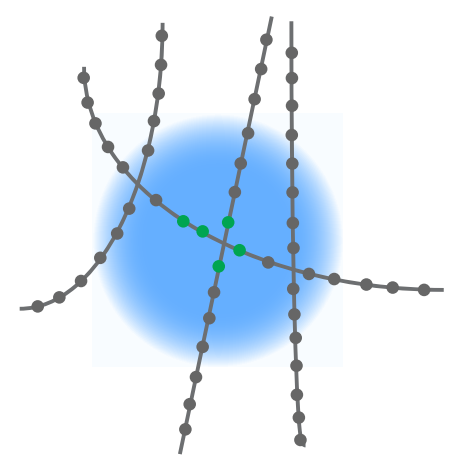

Figure 1.8: Imaging scheme of RESOLFT-microscopy utilizing negative switching RSFPs. A target structure (grey lines) is labelled with a negative switching RSFP (dots). At each scanning position of the image three illumination steps are repeated: 1. activation of the RSFPs at the scanning position, 2. offswitching of the RSFP in the periphery using a doughnut-shaped laser beam, 3. readout of fluorescence from the RSFPs which were located at the central position of zero intensity of the doughnut-shaped off-switching beam (green dots). The effective read-out area features a sub-diffraction size. The recorded fluorescence per scanning position is detected, the steps are then repeated at the next scanning position.

If a positive switching RSFP is used, the off-switching and readout steps can be combined if the off-switching step outweighs the on-switching of molecules in the periphery of the excitation volume [56].

The off-switching of molecules via the doughnut-shaped beam has to be saturated to guarantee a good state-separation at the readout time point and thus facilitate a sub-diffraction image. Therefore, the off-switching intensity and duration of the off-switching beam has to be chosen correctly. Further, the utilized RSFP needs to have a low switching background, i.e. low residual fluorescence in the off-switched state to enable a high resolution image.

Using the sub-diffraction effective PSF for scanning of the sample, a high-resolution image can be achieved. Therefore, the switching steps are performed at each scanning position of the sample and the fluorescence at each position is detected and reconstructed as an image. The light intensities used in scanning RESOLFTmicroscopy are in the range of $\sim 1-40 \mathrm{~kW} / \mathrm{cm}^{2}$, which is comparable to light intensities used in regular confocal microscopy [45], [60].

\subsection{Nanoscopy of Drosophila melanogaster}

Drosophila melanogaster is an important biological model system for developmental and cell biology. It is widely used as a simplified system to study cellular processes 
in living cells using live cell fluorescence microscopy. Drosophila features several valuable properties for microscopy, for example many of its tissues are relatively transparent and can be easily isolated by dissection. Mounted in the appropriate medium, the tissues can be kept viable for longer periods of time at room temperature to perform long term live cell fluorescence microscopy studies.

Large tissues however, can be challenging for fluorescence microscopy. Imaging in tissues is largely complicated by spherical aberrations caused by refractive index mismatches. Tissues feature many different cell types with a variety of optical properties. Different cell layers can posses different refractive indices. When imaging thick tissues, the excitation light is deteriorated by scattering, thereby reducing the quality and resolution of the image. Therefore, the refractive indices of the immersion media and the mounting media should be adapted to the respective tissue so as to reduce spherical aberrations (reviewed by [61]).

Drosophila melanogaster offers the possibility to label the protein of interest using genetically encoded fluorescent proteins via genetic modifications. Using spectrally different fluorescent proteins, several proteins of interest can be labelled and studied simultaneously. The protein of interest can be expressed as a fusion protein e.g. using the GAL4/UAS system [62]. Depending on the driver line, the expression of the fusion construct can be driven ubiquitously or restricted to a certain tissue or cell type. Using the CRISPR/Cas9 system, endogenously labelling is possible, thus avoiding artefacts induced by altering the expression level [59], [63].

Many studies report on the application of high resolution microscopy in Drosophila. Albeit most studies are based on the application of SIM-microscopy, which is limited to an achievable optical resolution of $\sim 120 \mathrm{~nm}$ [64]. SIM-microscopy is still diffraction-limited and does not perform a state separation within a diffractionlimited excitation volume to overcome the diffraction barrier, hence it is not a nanoscopy approach (Section 1.3).

There are only few studies regarding the application of nanoscopy methods in Drosophila melanogaster. These will be described in detail in the following.

In 2006, Kittel et al. reported on STED-microscopy of fixed presynaptic active zones, analysing the protein localization of Bruchpilot, an ERC/CAST family protein. This study showed ring-shaped substructures, which are centered at active zones of neuromuscular synapses [65]. Later, the studies were extended to multiple proteins located at the neuromuscular junction, including DLiprin-alpha and DSyd1, a Bruchpilot binding partner [66], [67].

Additionally to these biological findings using STED-microscopy, Drosophila melanogaster was used as a technical sample for demonstration of method develop- 
ment. In 2015, Jale et al. reported on STED-microscopy with a temporal resolution of $8 \mathrm{~ms}$ on EGFP labelled vesicles in neurons of living Drosophila larvae, leading to time- lapse recordings with a framerate of 125 frames per seconds [68].

Next to STED-microscopy, dSTORM was performed on fixed Drosophila tissues to perform quantitative imaging on active zones of neuromuscular junctions. It could be shown that the active zone cytomatrix comprises units built of $\sim 137$ clustered Bruchpilot proteins [69].

Quantitave 3D PALM was used to study the size distribution of E-cadherin clusters in fixed Drosophila embryos. Furthermore, it was shown that the E-cadherin cluster organization depends on interactions with the actin network as well as Dynamin depending endocytosis [70].

Recently, Structured Illumination Microscopy, Stimulated Emission Depletion microscopy and Single Molecule Localisation Microscopy were compared using fixed Drosophila macrophages and primary spermatocytes. SIM typically reached FWHMs of $\sim 107 \mathrm{~nm}$, while STED achieved a FWHM of $\sim 58 \mathrm{~nm}$ and STORM a FWHM of $\sim 56 \mathrm{~nm}$ on labelled microtubules [71].

As seen in these studies, the application of nanoscopy methods in Drosophila is almost exclusively based on chemical fixed samples. The fixation restricts the analysis to single snapshots of processes within the cell (e.g. protein localization); thus dynamic processes cannot be studied in real-time with high spatial resolution. The application of high resolution live cell RESOLFT-microscopy in Drosophila has not been analysed yet. Based on the low light levels applied in RESOLFT-microscopy, it could likely be suitable for high resolution time-lapse imaging in living animals and thus allow the characterisation of dynamic processes in Drosophila melanogaster for the first time.

\subsection{Nanoscopy of Arabidopsis thaliana}

The application of high-resolution fluorescence microscopy in plant science is complicated by the unique properties of plant material. Next to the plasma membrane, plant cells possess a thick cell wall consisting of several layers featuring different refractive indices, leading to light scattering during imaging. Further, the excitation light can excite light absorbing molecules located inside the plant cell, which can cause phototoxicity especially in photosynthesis active cells (reviewed in [72]).

Plant cells possess many sources of autofluorescence. One main source of autofluorescence are the chloroplast, which contain many light absorbing and emitting constituents like chlorophylls, carotenes, and xanthophylls. The cell walls include lignins, which emit fluorescence upon excitation at $\sim 530 \mathrm{~nm}[73]$. 
Reports on the application of nanoscopy methods in living plant cells are restricted to two studies which will be described in the following.

Using STORM, the cellulose microfibril organization in onion cells was analysed [74]. Next to this, there is one report of the application of STED-microscopy in living plants. In this study YFP-labelled PIN proteins at the apical plasma membrane of living root cells of Arabidopsis thaliana were analysed. However, the study was restricted to non-photosynthesis active cells, making phototoxic effects less likely [75]. The absence of a large number of live-cell high-resolution studies in plant cells is likely explained by their challenging properties in fluorescence microscopy. Every high-resolution microscopy method has some limitation which make its application in plant cell imaging challenging.

The high light intensities applied in STED-microscopy make this method unfavourable for imaging in photosynthesis active cell types. The red-shifted depletion laser can be absorbed by the chlorophylls and lead to a local heating of the cell [76]. Further studies on the application of STED-microscopy are needed to analyse its usability in a broad set of cell types of different plant species as well as to further determine the photostress induced by the light intensities applied in STEDmicroscopy.

The application of stochastic methods (STORM, PALM) for high-resolution plant imaging is mostly restricted to studies using fixed samples, due to the time consuming imaging scheme. For the reconstruction of a single high resolution image several hundred to thousand single frames were recorded, which typically results in acquisition times of several minutes per single image [60]. If the labelled structure is moving while the single frames are recorded, reconstruction artefacts might occur in the post-processing of the recorded data.

Time-lapse imaging of cellular dynamics in planta have not yet been demonstrated. High-resolution RESOLFT-microscopy requires only low light intensities of $\sim 1$ $40 \mathrm{~kW} / \mathrm{cm}^{2}$ to achieve a sub-diffraction resolution image [45]. Hence RESOLFTimaging is likely suited for imaging in light sensitive plant cells. However, a demonstration of RESOLFT-microscopy for high resolution imaging of plant cells is still missing. Therefore the usability of RESOLFT-microscopy for imaging in plant cells is characterized in this work. 


\subsection{Aim of the study}

The relatively low light intensities used in RESOLFT-microscopy make it a very suitable high-resolution microscopy method for imaging dynamical processes in living samples. Nevertheless, all RESOLFT demonstrations so far were restricted to single cells. A report of the application of RESOLFT imaging for imaging in multicellular organisms and for in vivo studies is still required.

This work focuses on the characterisation and establishment of high resolution RESOLFT-microscopy of multicellular organisms for the first time.

In the first part of the thesis, the usability of RESOLFT-microscopy for high resolution imaging of tissues and for in vivo imaging of the multicellular organism Drosophila melanogaster is characterized.

In the second part of the thesis the applicability of high resolution RESOLFT- and STED-microscopy for imaging in light absorbing plant cells is analysed for the first time. 


\section{Results}

\subsection{RESOLFT-microscopy of Drosophila melanogaster}

To analyse and adapt the usability of RESOLFT-microscopy for in vivo imaging, Drosophila melanogaster was chosen as a multicellular model organism. The possibility to perform long-term imaging at room temperature makes it the ideal model system for the establishment of in vivo-RESOLFT imaging.

\subsubsection{Generation of stably expressing rsEGFP2- $\alpha$-tubulin Drosophila melanogaster}

To label the microtubule cytoskeleton within cells of Drosophila melanogaster, a fusion construct consisting of rsEGFP2 fused to the N-terminus of $\alpha$-tubulin (CG1913) was generated (Section 4.1). Strong overexpression of a fusion protein can lead to artefacts within the target structure and influence the viability of the cell (reviewed by [58]). Therefore a strong overexpression was avoided and a permanent expression at relatively low levels was chosen. The artificial expression of the fusion protein is driven ubiquitously by the promotor region of the ubiquitin 68E gene (CG11624) in addition with the endogenous non-labelled $\alpha$-tubulin protein. This expression construct was integrated into the landing site $3 \times \mathrm{RFP}-86 \mathrm{fb}$ located on the third chromosome and crossed homozygous resulting in a stably expressing rsEGFP2- $\alpha$ tubulin fly stock (Section 4.1). This stock was used in all following experiments and is denoted as rsEGFP2- $\alpha$-tubulin.

\subsubsection{Characterisation of rsEGFP2- $\alpha$-tubulin expression}

To characterise the expression of the generated rsEGFP2- $\alpha$-tubulin stock, tissues from different developmental stages were isolated and analysed via live-cell fluorescence microscopy. The results of the expression studies in selected tissues are shown in Figure 2.1. The microscopic images of several different tissues confirm the expression of the rsEGFP2- $\alpha$-tubulin construct in all tissues analysed (Figure 2.1). The characterisation revealed a large variation of the morphology of the microtubule cytoskeleton in different cell types.

In body wall muscles, the tubulin filaments show an alignment along the muscle fibers (Figure $2.1 \mathrm{a}$ ). In salivary glands, the signal to noise ratio is lower compared to body wall muscles but the microtubules build a dense network (Figure $2.1 \mathrm{~b}$ ). 

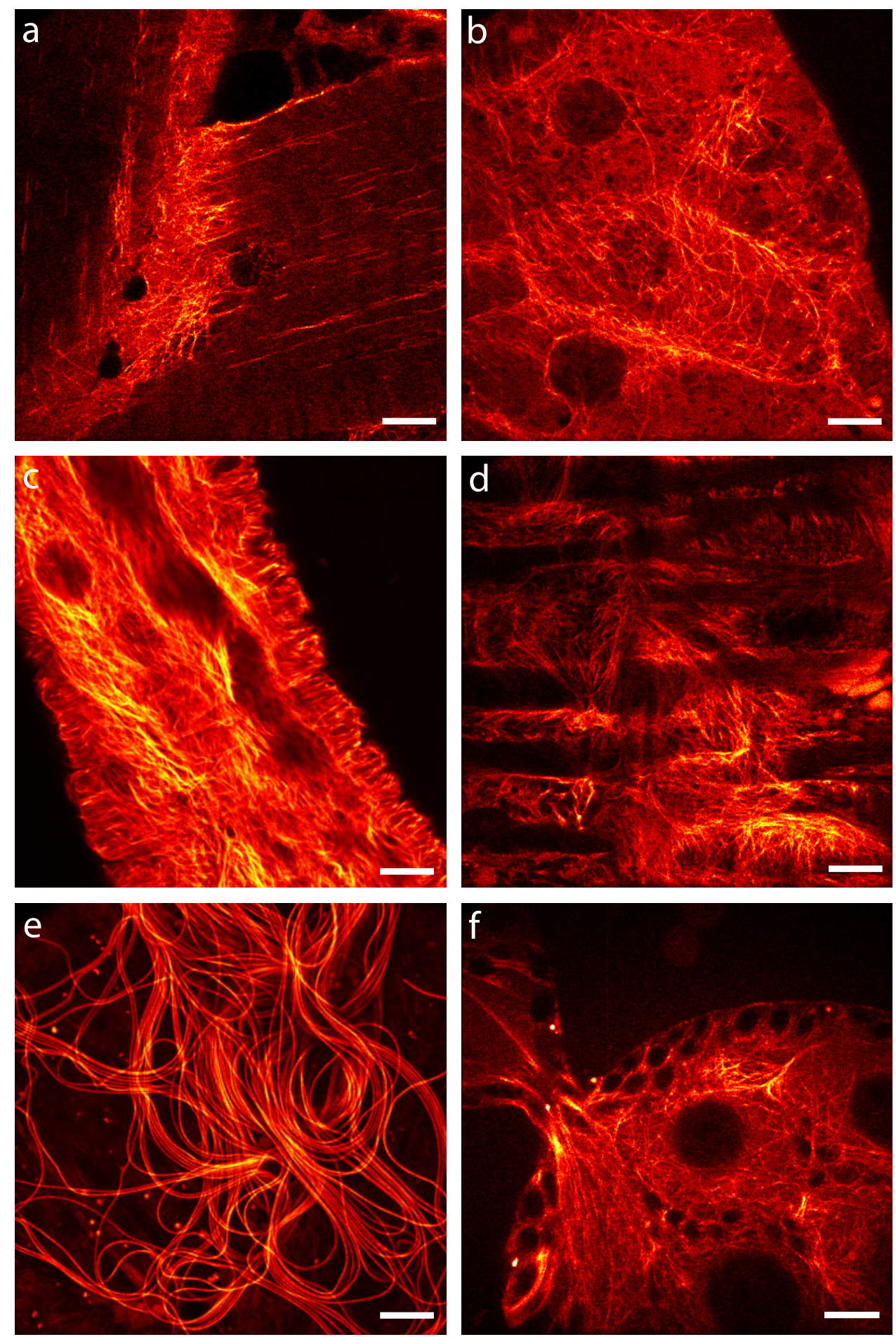

Figure 2.1: Characterisation of rsEGFP2- $\alpha$-tubulin expression pattern in living resected tissues. Confocal fluorescence microscopy recordings of living resected tissues of transgenic wandering third instar larvae (a-d) and of adult flies (e, f) ubiquitously expressing rsEGFP2- $\alpha$-tubulin. (a) Body wall attachment site, (b) salivary glands, (c) salivary duct, (d) intestinal muscles, (e) sperms, (f) ovaries. Images display raw data. Scale bars: $10 \mu \mathrm{m}$ (modified after [77]). 


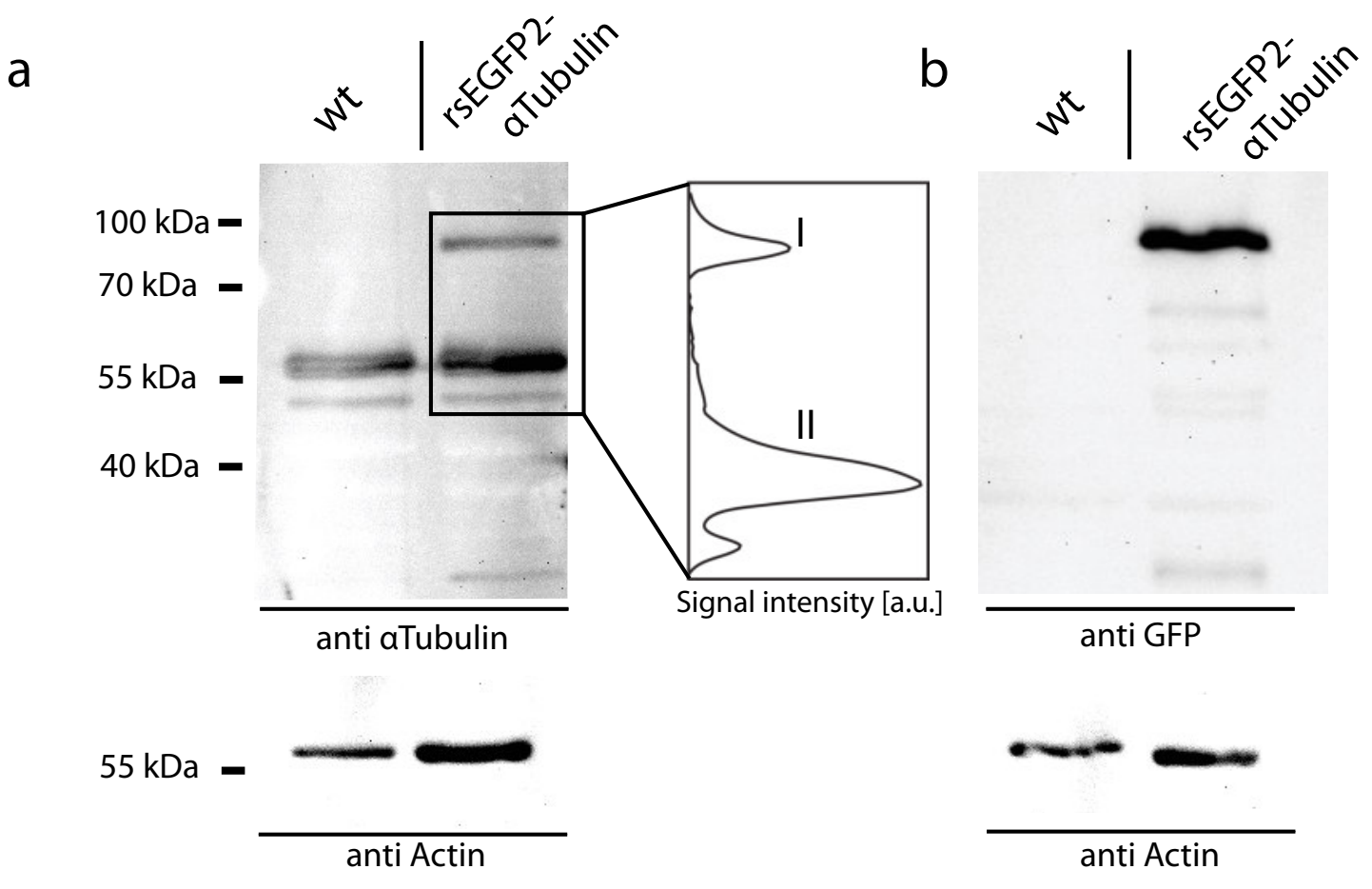

Figure 2.2: Western blot analysis of the expression level of rsEGFP2- $\alpha$ tubulin in Drosophila melanogaster third instar larvae. Protein lysates of dissected Oregon R (wild type control) and rsEGFP2- $\alpha$-tubulin expressing Drosophila melanogaster larvae were decorated with antisera against a) $\alpha$-tubulin and b) GFP. As a loading control, the blots were decorated with an antiserum against actin. To analyse the ratio between labelled (peak I) and non-labelled (peak II) $\alpha$ tubulin signal in a) the respective signal was determined using the ImageJ plugin Gel Analyzer. The integral of the peaks for labelled (peak I) and non-labelled (peak II) $\alpha$-tubulin was determined and the ratio was calculated (Section 5.2) (modified after [77]).

In the salivary duct different orientations of the tubulin filaments of different cell layers becomes apparent in the optical section (Figure $2.1 \mathrm{c}$ ). At the outside of the intestine, single muscle strips are visible, which are surrounded by cells featuring a strong labelled microtubule network (Figure $2.1 \mathrm{~d}$ ). As depicted in Figures $2.1 \mathrm{e}$ ) and f), also the microtubule cytosceleton within germline cells (sperms and oocytes) are labelled by the expression of the rsEGFP2- $\alpha$-tubulin construct. The labelling of the microtubule filaments within the germline demonstrates the versatility of rsEGFP2 as a genetically encoded fluorescent protein for tagging. To analyse whether the labelling of the microtubule network impacts the fitness of the rsEGFP2- $\alpha$-tubulin expressing flies, the average life span of the flies was analysed. 
The flies had a life span of $\sim 41$ days, similar to the isogenic wild type $w^{1118}$ strain which was used in this experiment as reference (Section 4.5). These results demonstrate that the generated rsEGFP2- $\alpha$-tubulin expressing fly stock showed expression in all tissues analysed, while the flies were not influenced in their fitness.

To determine the ratio between rsEGFP2-labelled and non-labelled endogenous $\alpha$ tubulin subunits, protein lysate from wandering third instar larva was analysed by Western blot (Section 4.3). Due to the fusion of rsEGFP2 to the $\alpha$-tubulin subunit, the increased molecular weight of this fusion protein can be used to differentiate between the labelled and non-labelled $\alpha$-tubulin subunits.

As depicted in Figure 2.2, the protein lysate of rsEGFP2- $\alpha$-tubulin expressing larvae show an additional signal at molecular weight of $\sim 90 \mathrm{kDa}$. Probing with an antiserum against GFP showed a signal of similar molecular weight, indicating that this signal corresponds to rsEGFP2- $\alpha$-tubulin.

Probing against $\alpha$-tubulin as well as against GFP showed small degree of degradation of the fusion construct within the protein lysates. The signal intensities between the rsEGFP2-labelled and the unlabelled $\alpha$-tubulin show a 3:1 ratio (Section 5.2). Following this, approximately one out of four $\alpha$-tubulin subunits are labelled. During the whole study, no signs of artefacts in the morphology of the microtubule filaments were observed.

Altogether, the generated homozygous ubiquitously rsEGFP2- $\alpha$-tubulin expressing fly stock showed a specific labelling of the microtubule network in various cell types, while not influencing the fitness of the flies.

\subsubsection{RESOLFT-microscopy of dissected tissues}

\subsubsection{2D-RESOLFT imaging of living tissues}

To examine the applicability of RESOLFT-microscopy for high resolution imaging of Drosophila melanogaster, several dissected larval tissues were used as sample for the imaging. The tissues were maintained in Schneider's cell culture medium to keep them under physiological buffer conditions.

For RESOLFT imaging, switching parameters had to be identified to establish RESOLFT imaging using rsEGFP2 in tissues of Drosophila melanogaster. Different tissues showed varieties in the signal to noise ratio. Therefore pump and probe measurements were performed to identify optimal RESOLFT parameters for each individual tissue to achieve the best possible RESOLFT image. All laser intensities used in this work, represent the light intensities measured at the position of the objective's back focal plane. 
All microscopic images in this chapter display only raw data to fully report on the possibilities as well as on the limitations of RESOLFT-microscopy using rsEGFP2 in Drosophila melanogaster. To compare the resolution enhancement in the RESOLFT images, a diffraction-limited confocal image at the same position was recorded with similar imaging parameters except performing the off-switching step (Section 1.4.4). In the following, single examples of RESOLFT imaging in different tissues are shown. The achieved resolution was analysed by determining the FWHM of the line profiles measured across single tubulin filaments. To exclude mistakes in the measurement by the occurrence of statistical outliers in the signal intensity, all line profiles were averaged over the extent of three (Figures 2.3, 2.4, 2.5, 2.10), or five (Figure 2.9) or ten pixels (Figure 2.7, 2.12).

For RESOLFT imaging of dissected salivary glands, the recording sequence was as following: first the rsEGFP2 proteins were switched into the fluorescent on-state by irradiation with $405 \mathrm{~nm}$ light $(4.1 \mu \mathrm{W})$ for $20 \mu \mathrm{s}$. After a short illumination break of $5 \mu \mathrm{s}$, the off-switching $488 \mathrm{~nm}$ doughnut shaped beam $(28 \mu \mathrm{W})$ was applied for 430 $\mu$ s to switch the rsEGFP2 molecules in the periphery of the excitation volume to the fluorescent off-state. Subsequently, a second Gaussian shaped laser beam of $488 \mathrm{~nm}$ $(11.9 \mu \mathrm{W})$ was used for $30 \mu$ s to read out the fluorescence of the residual rsEGFP2 molecules in the fluorescent on-state at the center of the excitation volume. These three steps of illumination were performed at each scanning position, i.e. at every pixel of $25 \mathrm{~nm}$ width. To increase the signal to noise ratio, every line was scanned twice and the signal per line was accumulated. The RESOLFT image of salivary glands is depicted in Figure $2.3 \mathrm{a}$ ) and b).

The microtubule network in salivary glands showed a high degree of branching and interconnections between individual filaments. By applying the RESOLFT imaging mode, these single individual filaments become visible and are now separable. The resolution enhancement is supported by the line profiles which were taken across single tubulin filaments (Figure $2.3 \mathrm{~b}$ )). Here a FWHM of $\sim 50 \mathrm{~nm}-60$ $\mathrm{nm}$ is achieved, this represents an improvement in resolution by a factor of $\sim 4-5$ compared to the diffraction-limited resolution in the corresponding confocal image. In the confocal image, this degree of branching and interconnections is not visible. Next, RESOLFT images of rsEGFP2-labelled tubulin filaments in dissected body wall muscles were recorded. Imaging was performed within the first cell layer, therefore the intensity of the activation pulse of $405 \mathrm{~nm}$ could be decreased to $1.5 \mu \mathrm{W}$. After on-switching using light of $405 \mathrm{~nm}(1.5 \mu \mathrm{W})$ for $17 \mu \mathrm{s}$, the off-switching of molecules in the periphery was performed by illumination with $26 \mu \mathrm{W}$ for $380 \mu \mathrm{s}$ with the doughnut shaped $488 \mathrm{~nm}$ beam. A short $(10 \mu \mathrm{s})$ strong $(14.4 \mu \mathrm{W})$ 

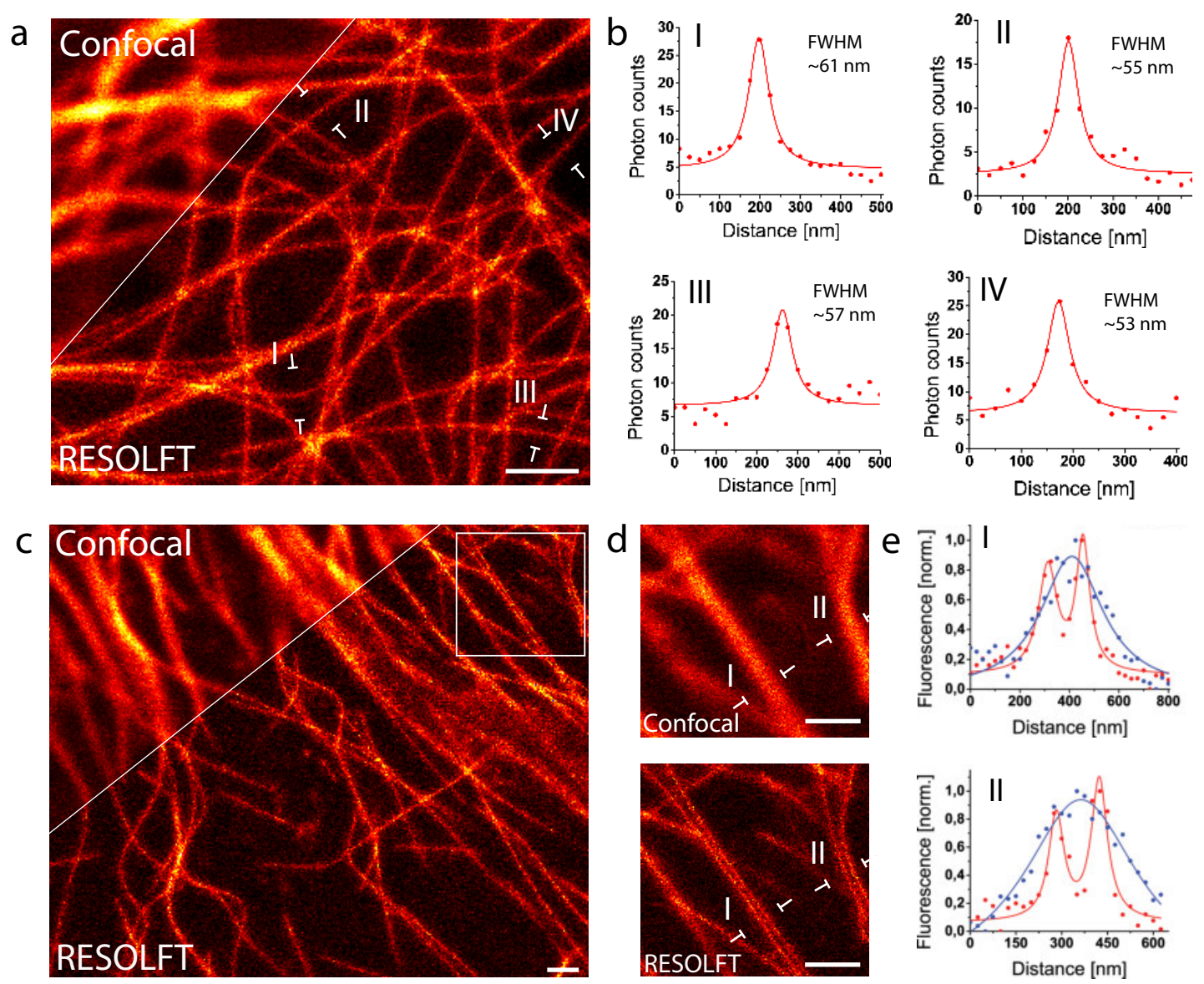

Figure 2.3: RESOLFT-microscopy of resected salivary glands and body wall muscles of third instar larvae. (a) Comparison of confocal and RESOLFT recordings taken on salivary glands of a larva expressing rsEGFP2- $\alpha$-tubulin. b) Line profiles averaged over three pixel sizes across single filaments at positions indicated in (a) demonstrating a FWHM below $\sim 60 \mathrm{~nm}$. c) Confocal and RESOLFT image of resected body wall muscle. d) Confocal and RESOLFT close ups of area indicated in (c). e) Line profiles accros three pixel sizes of indicated positions in d). Blue lines indicate Line profiles of confocal image, whereas the red line indicates the line profile of the RESOLFT image. All images display raw data. Scale bars: $1 \mu \mathrm{m}$ (modified after [77]).

read-out pulse resulted in a good signal to noise ratio in the RESOLFT image. Each scanning line was recorded twice with $20 \mathrm{~nm}$ pixel size and the recorded signal was accumulated to obtain the final image (Figure $2.3 \mathrm{c}$ ). In body wall muscles, the microtubules are often co-aligned over several micrometer distance. In the diffraction-limited confocal image, these filaments can not be separated and appear as one filament (Figure $2.3 \mathrm{~d}$ ). In the high-resolution RESOLFT image, these filaments can be optically separated. This can be seen in the corresponding line-profiles of the RESOLFT (red-line) and confocal (blue-line) images (Figure 2.3 e). 


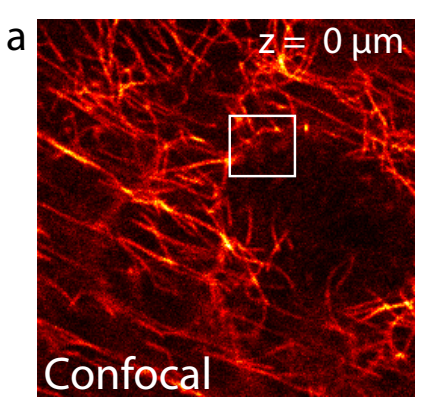

d

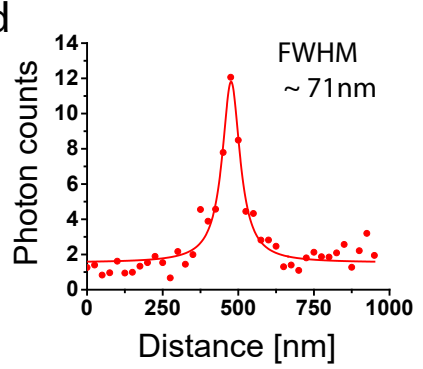

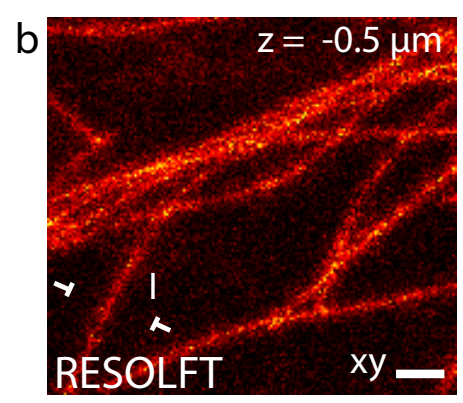

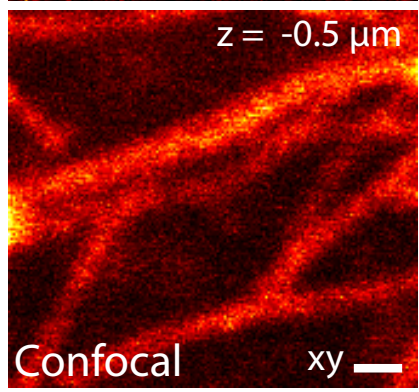

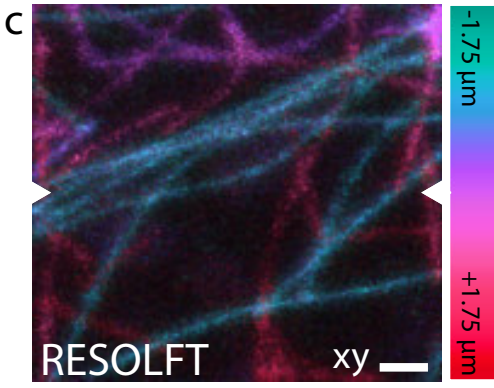

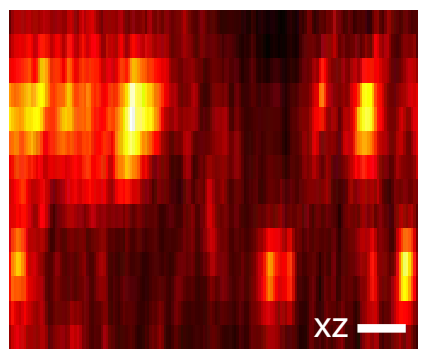

Figure 2.4: RESOLFT volume imaging using a 2D-doughnut for offswitching. Dissected living body wall muscles of a Drosophila melanogaster larva ubiquitously expressing rsEGFP2- $\alpha$-tubulin were imaged. Along $3.5 \mu \mathrm{m}, 14$ RESOLFT images were recorded with $250 \mathrm{~nm}$ axial distance between each image.

a) Confocal overview at the center plane $(\mathrm{z}=0 \mu \mathrm{m})$ of the RESOLFT image stack.

b) RESOLFT and corresponding confocal image of the $3 \mathrm{D}$ stack recorded at $\mathrm{z}=$ $-0.5 \mu \mathrm{m}$. c) top: Maximum intensity projection of the recorded volume displayed in the xy-view. The color coding shows the relative z-position of the microtubule filaments within the recorded volume, bottom: xz slice of indicated position at white arrowheads in c). d) line profile taken at indicated position in b). a), b) display raw data, c) top: displays color coded data smoothed with 1.2 pixel wide Gaussian, bottom: displays data smoothed with 1.2 pixel wide Gaussian. Scale bars: $2 \mu \mathrm{m}$ a), and $500 \mathrm{~nm} \mathrm{~b}$ ), c) (modified after [77]).

Together, the performed RESOLFT imaging of various tissues showed a resolution of $50 \mathrm{~nm}-60 \mathrm{~nm}$ this represents a resolution enhancement by a factor of $\sim 4-5$ times compared to the corresponding diffraction-limited confocal images. The chosen tissues depicted in Figure 2.3 are a selection of a variety of different tissues which were recorded with high-resolution in the RESOLFT mode.

The improved resolution in RESOLFT-microscopy enabled the visualization of fine sub-features of the microtubule network in living cells, like branching and co-alignment which are unattainable by diffraction-limited regular confocal microscopy. The axial resolution is still diffraction-limited, because the utilized off-switching doughnut-shaped beam only leads to a confinement of molecules remaining in the fluorescent on-state within the lateral plane. 
By performing volume imaging (z-stacks) in cells, the three dimensional arrangement of filaments can be analysed. In Figure 2.4 this is exemplified, here a z-stack is recorded in the RESOLFT mode utilizing a two-dimensional off-switching doughnutshaped beam. The xy-pixel size was set to $25 \mathrm{~nm}$ to fulfil the Nyquist criterion to display a resolution of $70 \mathrm{~nm}$. To analyse the three dimensional arrangement of the microtubule network, 14 RESOLFT images with $250 \mathrm{~nm}$ axial distance between each image were recorded, resulting in a recorded volume of $4.22 \mu \mathrm{m} \times 3.9 \mu \mathrm{m}$ $\mathrm{x} 3.5 \mu \mathrm{m}$. The individual frames show the resolution enhancement along the lateral plane featuring a FWHM of $\sim 71 \mathrm{~nm}$ (Figure $2.4 \mathrm{~b}$ ), d)). The 14 individual RESOLFT images were reconstructed as a maximum intensity projection which is color coded in respect with the imaging depth of each frame visualizing the three dimensional arrangement within the recorded volume (Figure 2.4 c). Single xz slices demonstrate the diffraction-limited resolution along the axial axis. However, the three dimensional arrangement of filaments within the recorded volume can still be visualized.

\subsubsection{3D-RESOLFT imaging of living tissues}

To follow individual microtubule filaments along all three room dimension with high-resolution, the xy-off-switching doughnut was exchanged against an xyz-offswitching light pattern by integrating a spatial light modulator in the microscope (Section 4.10). The three dimensional off-switching pattern restricts the activated rsEGFP2 molecules along all spatial directions to a sub-diffraction sized volume, thus improving the resolution along the $\mathrm{x}, \mathrm{y}$ and $\mathrm{z}$ axes.

In the 3D-RESOLFT mode, a volume of $8.5 \mu \mathrm{m} \times 6.8 \mu \mathrm{m} \times 1.98 \mu \mathrm{m}$ was recorded by capturing 33 single RESOLFT xy-frames with an axial distance of $60 \mathrm{~nm}$ between each image. To record the three dimensional volume in the RESOLFT mode, several imaging parameters had to be optimized to compensate the bleaching of rsEGFP2 during the image acquisition. Due to the fact that the PSF of the individual onand off-switching lasers have an axial size of $\sim 600 \mathrm{~nm}$, up to ten xy-slices with a thickness of $60 \mathrm{~nm}$ were illuminated, while only one slices was actually recorded. This led to the cycling of the rsEGFP2 molecules between the fluorescent on- and off-state and therefore to switching fatigue (bleaching) before these molecules were recorded within their respective slice. To reduce the bleaching during imaging, the applied light intensities were reduced (405 nm activation: $2.9 \mu \mathrm{W}$ and $488 \mathrm{~nm}$ readout: $10.2 \mu \mathrm{W})$. In addition, the pixel size in the lateral plane was set to $35 \mathrm{~nm}$ and no line accumulation step was performed. 

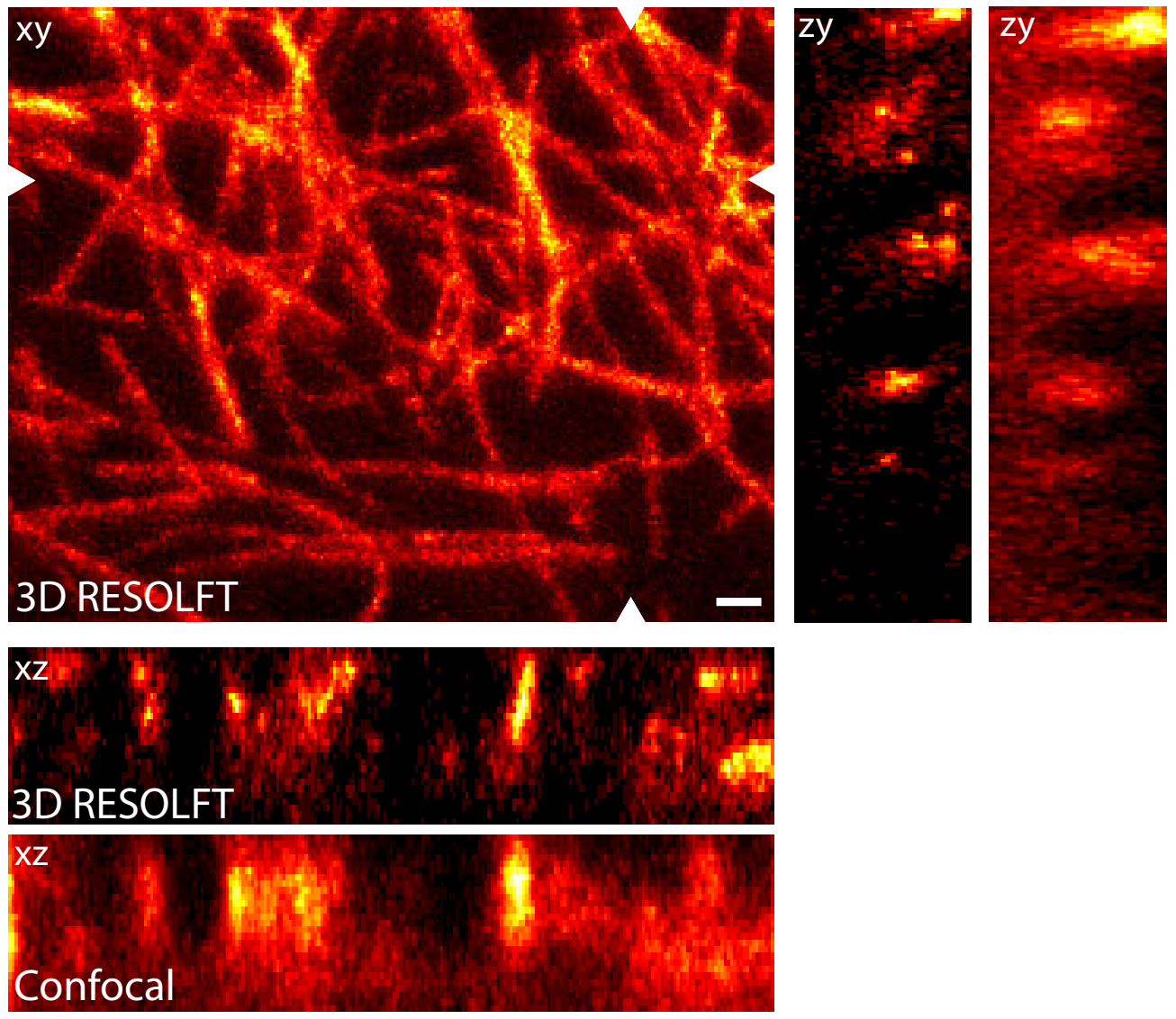

Figure 2.5: RESOLFT imaging of volumes using a 3D-light pattern for off-switching. Dissected living body wall muscles of a Drosophila melanogaster larva ubiquitously expressing rsEGFP2- $\alpha$-tubulin were imaged. The recorded volume features a size of $8.5 \mu \mathrm{m} \times 6.8 \mu \mathrm{m} \times 1.98 \mu \mathrm{m}$. a) Large image: Maximum intensity projection of the RESOLFT image stack in the xy view. Smaller images: single xz-, zy-slices from the indicated positions (arrowheads) of the maximum intensity projection. RESOLFT stack was recorded in indicated area of b). b) Confocal overview image of living body wall muscle. c) large image: false color depth coding of the maximum intensity projection to visualize the three dimensional arrangement of filaments in the recorded volume. smal images: single xz-, zy-slices from the indicated positions (arrowheads) of the maximum intensity projection. d) Line profiles along indicated positions in c). Images display raw data smoothed with 1.2 pixel wide Gaussian. Scale bars: $1 \mu \mathrm{m} \mathrm{a}$ ), and $500 \mathrm{~nm} \mathrm{~b}$ ), c) (modified after [77]).

To illustrate the three dimensional arrangement of the dense microtubule network, the recorded high-resolution images were projected as a maximum intensity projection and color-coded with respect to the corresponding imaging depth of each recorded RESOLFT frame (Figure $2.5 \mathrm{c}$ ). The improvement in resolution along the axial direction is exemplified in single xz- and zy-slices of the recorded volume, showing an $\sim 4$ fold improvement in resolution along the z-axis (Figure $2.5 \mathrm{~d}$ ). In the recorded volume single microtubule filaments are traceable along all spatial di- 
rections. This is exemplified by the selected xz slice in Figure $2.5 \mathrm{c}$ ), which shows one filament crossing the whole recorded axial distance. The line profile along the indicated position depicts a FWHM of the filament of $\sim 160 \mathrm{~nm}$. The resolution enhancement along the axial axis allows the optical separation of filaments in the cross-section that are located closely above each other (indicated by an arrow in xz slice in Figure 2.5 a).

RESOLFT imaging of dissected tissues of Drosophila melanogaster is feasible featuring a resolution of $50 \mathrm{~nm}-60 \mathrm{~nm}$ along the lateral axis (Figure 2.3). By utilizing a three-dimensional off-switching pattern, RESOLFT recording of volumes within tissues with increased resolution along the axial axis is possible. Here a resolution of $\sim 140 \mathrm{~nm}$ along the axial axis could be achieved, this represents a 4 fold resolution enhancement compared to regular confocal resolution (Figure 2.5).

\subsubsection{RESOLFT-microscopy at different imaging depths}

All images so far were recorded within the first cell layers of isolated tissues at a distance of $\sim 1-10 \mu \mathrm{m}$ from the coverslip. A confocal scanning microscope offers optical separation of the fluorescence which originates from the focal plane and the background from other planes. The rejection of out-of-focus light is crucially important in highly fluorescent tissues to enable a good signal to noise ratio in the image. By removing the pinhole of the microscope the recorded image largely reflects a wide-field image. By integrating the pinhole in the detection path, a confocal counterpart image can be recorded. The excitation is still realized by a scanning point-like excitation spot, but the loss in image quality is discernable in comparison to a confocal image recorded using a pinhole in the detection path (Figure 2.6). The single filaments within the dense microtubule network in the tissue, can only be discerned using a confocal detection. The depicted image was recorded within the first cell layer. The background noise will increase if the imaging is performed deep within the tissue. By using a confocal scanning microscope, imaging in highly fluorescent tissues is not restricted to the first cell layers.

The ability to reject out of focus light in a confocal microscope becomes important in tissue imaging at larger image depth. By focussing into a tissue, the excitation light is scattered by the tissue and the difference in refractive indices lead to spherical aberrations, which will decrease the signal intensity from the focus. The scattered light also excites molecules outside of the focus, thus increasing the background noise. Confocal detection can reduce this background noise, but with increasing imaging depth the signal to noise ratio becomes worse. 
a
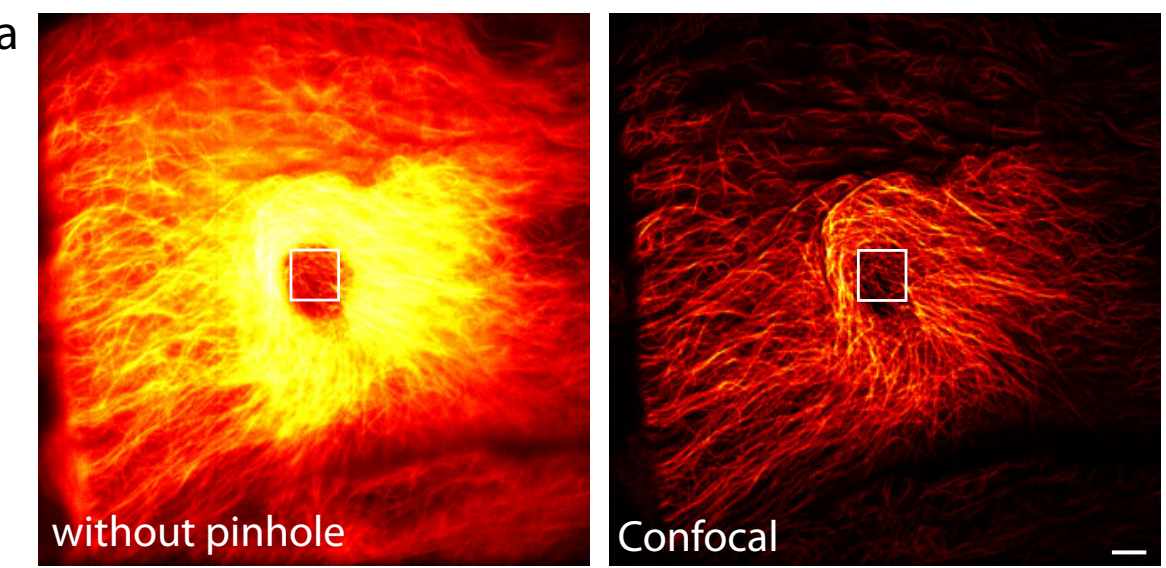

b
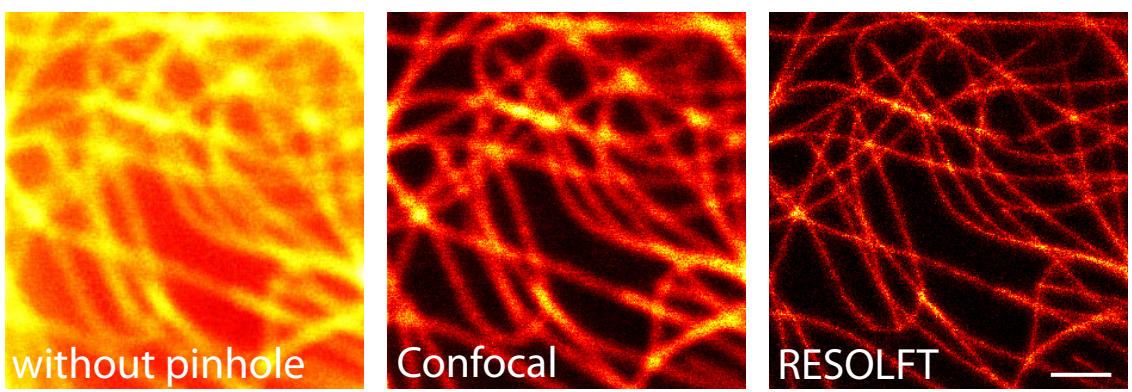

Figure 2.6: Image quality of confocal detection in tissues of Drosophila melanogaster. Fluorescence images of living dissected third instar larva body wall muscles expressing rsEGFP2- $\alpha$-tubulin. Pictures were recorded without, with a pinhole (size: 1 Airy unit) and in the RESOLFT mode (pinhole: 1 Airy unit). Boxed area in a) is magnified in b). All images display raw data. Scale bars: a): 5 $\mu \mathrm{m}, \mathrm{b}): 1 \mu \mathrm{m}$.

To analyse whether RESOLFT imaging can reduce the increasing background noise by utilizing the photo-switching feature of the rsEGFP2 molecules, RESOLFT imaging was performed at different imaging depths.

To evaluate the strength of 2D-RESOLFT imaging within highly fluorescent tissues, RESOLFT-images were recorded at different depths using the same imaging parameters. To generate a thick fluorescent sample, the front half of a wandering third instar larva was inverted and mounted in Schneider cell culture medium. The first image was recorded at the first cell layer of body wall muscles, while the last images were recorded up to $40 \mu \mathrm{m}$ deeper within the sample. The microscope was equipped with an objective lens which used silicon oil as the immersion medium of refractive index $n=1.406$ to better match the refractive index of the tissue reducing spherical abberations. 

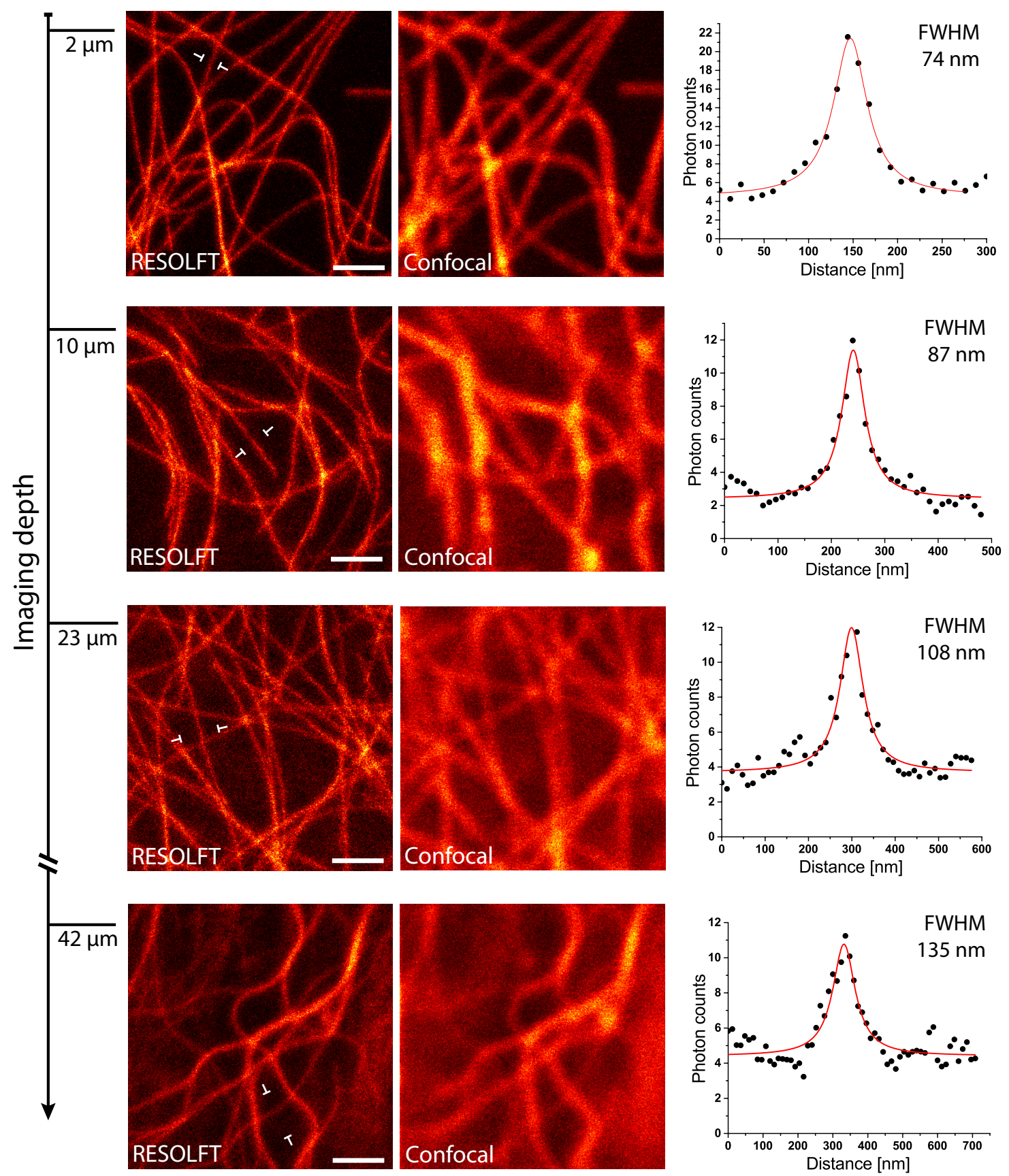

Figure 2.7: RESOLFT imaging at different imaging depths. RESOLFT and corresponding confocal images were taken at the indicated depths on body wall muscles of a dissected third instar larva ubiquitously expressing rsEGFP2- $\alpha$ tubulin. Line profiles were taken at the indicated positions across single filaments in the RESOLFT frame. The data points represent an average of ten adjacent (20 $\mathrm{nm}$ distance between each measurement) measurements. The averaged data were fitted with a Lorentzian function (solid line). The FWHM was determined on the fitted function. All images display raw data. Scale bars: $1 \mu \mathrm{m}$ (modified after [77]).

As expected, the signal to noise ratio decreased upon focusing deeper into the highly fluorescent tissue (Figure 2.7). At depths of $10 \mu \mathrm{m}$ and $23 \mu \mathrm{m}$ the confocal images 
show increasing background noise, while the signal intensity is decreasing. In the RESOLFT mode, individual filaments can be resolved with improved optical resolution of $87 \mathrm{~nm}$ and $108 \mathrm{~nm}$ as well as with better signal to noise ratio compared to the confocal counterpart images. At an imaging depth of $42 \mu \mathrm{m}$, the achieved resolution in the RESOLFT mode is decreasing to $135 \mathrm{~nm}$ while the signal to noise ratio is increasing to $40 \%$.

The RESOLFT images at higher imaging depths illustrate the contrast enhancement by utilizing the switching capability of RSPFs, which was described previously [78], [79].

\subsubsection{Time-lapse RESOLFT-imaging}

To display the dynamic growth and depolymerization of single microtubule filaments in high-resolution, dissected body wall muscles were imaged continuously in the RESOLFT-mode over 40 frames. To capture the highly dynamic behaviour of the filaments, the imaging parameters had to be adapted to decrease the acquisition time of a single RESOLFT frame. Therefore the pixel size was set to $35 \mathrm{~nm}$ and the off-switching step was shortened to $180 \mu \mathrm{s}$. The shortening of the off-switching step was compensated by increasing the laser intensity of the off-switching beam to $\sim 40 \mu \mathrm{W}$ to keep the resolution improvement comparable to Figure 2.4. The modified imaging parameters enabled the recording of a single RESOLFT frame of $52 \mu \mathrm{m}^{2}$ size within 9.3 seconds. As depicted in Figure 2.8, the microtubule filaments display fast dynamic behaviour. Between the individual frames, growing as well as depolymerization of single filaments can be observed.

When recording multiple RESOLFT images of the same region of interest, switching fatigue of the RSFPs can lead to a decrease in image quality as well as declining resolution over the individual frames.

To visualize the reduction in signal intensity through bleaching, the colormaps of the depicted frames in Figure 2.8 are normalized to the maximum signal of the first frame. The decrease in signal intensity of single filaments is hereby directly reporting the bleaching of the fluorescent proteins during imaging. As can be seen in Figure 2.8, over 40 RESOLFT frames the fluorescent signal of the labelled microtubule filaments is bleaching. Nevertheless, the recorded time-lapse RESOLFT imaging allows the characterisation of the dynamic changes of the microtubule network over 40 RESOLFT frames over a total observation time of $\sim 6$ minutes at high spatial resolution. Beside the discernible bleaching in the recorded frames, no obvious sign of reduced polymerization of filaments or cell damage induced by the continuous imaging were visible. 

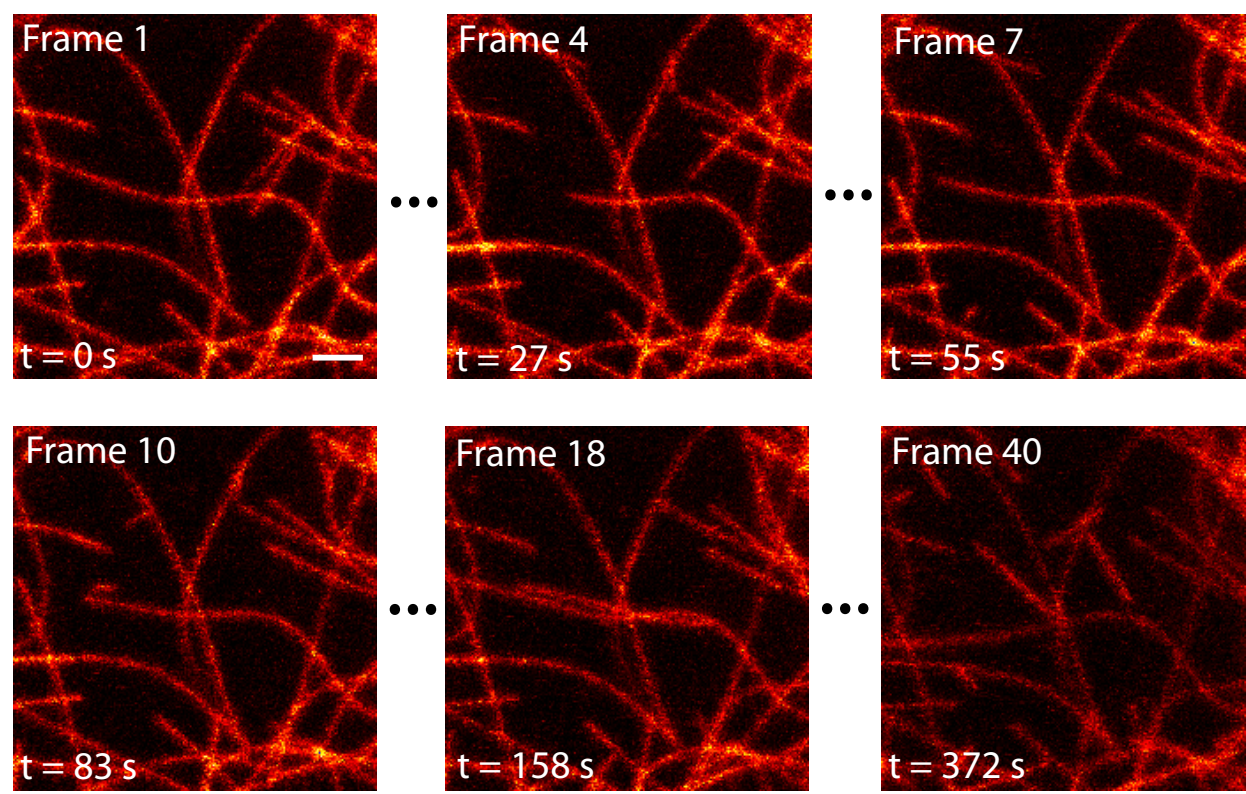

Figure 2.8: Time-lapse RESOLFT imaging with high frame rate of the microtubule cytoskeleton in body wall muscles of a dissected Drosophila melanogaster larva. Selected frames from in total 40 frames are displayed. The colormap is normalized to the brightness of the first frame. Recording time of a single frame: 9.3 seconds. All images display raw data. Scale bar: $1 \mu \mathrm{m}$ (modified after [77]).

To further analyse if the light intensities used in RESOLFT-microscopy are influencing the cell viability during image acquisition, a long-term RESOLFT movie was recorded. Here an area of $552 \mu \mathrm{m}^{2}$ of a body wall muscle of a dissected third instar larva was recorded. By recording 40 frames with an acquisition time of $\sim 4$ minutes per frame, the region of interest was imaged continuously for $\sim$ 2.5 hours. The RESOLFT imaging parameters were set to a pixel size of $30 \mathrm{~nm}$ without performing line accumulation. The off-switching doughnut-shaped beam was applied at each scanning position for $300 \mu \mathrm{s}$ at $39 \mu \mathrm{W}$. By applying these image parameters, a resolution of $\sim 73 \mathrm{~nm}$ was achieved, demonstrated by the line profile taken across five pixels at the indicated position within the first RESOLFT frame.

Before and after the long-term RESOLFT image acquisition at the same position, confocal overview images were taken to visualize potential signs of cellular distress in the imaged area as well as in the surrounding area (Figure $2.9 \mathrm{c}$ ). 

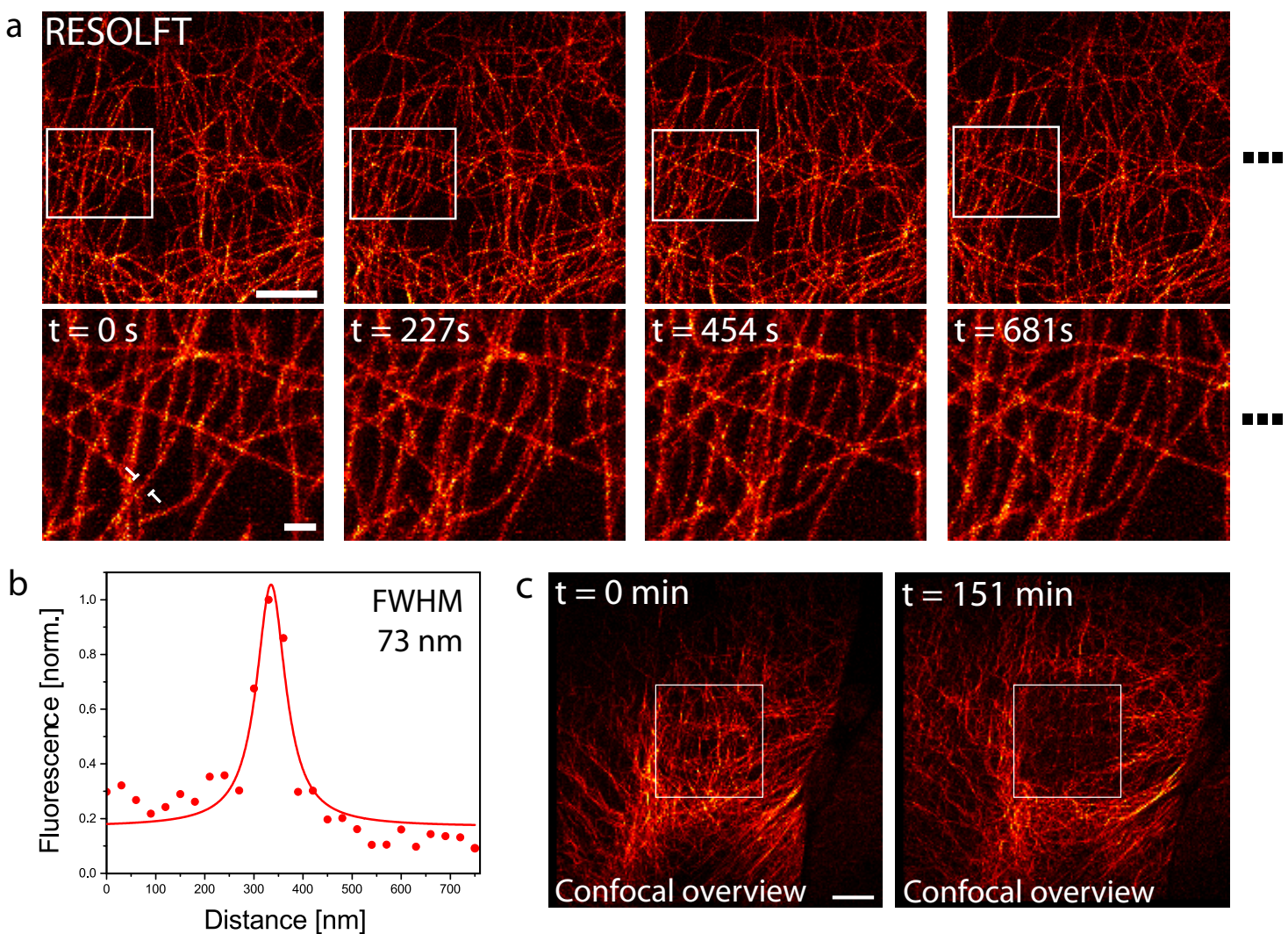

Figure 2.9: Time-lapse RESOLFT imaging of the microtubule cytoskeleton in body wall muscles of dissected Drosophila melanogaster larva expressing rsEGFP2- $\alpha$-tubulin. a) 4 selected frames from in total 40 frames are displayed. Upper row: recorded RESOLFT frames, lower row: magnifications of areas indicated in corresponding upper images. Recording time of a single frame: 227 seconds. b) line profile taken across single tubulin filament at the indicated position in a). The datapoints represent an average of five adjacent measurements. The averaged data were fitted with a Lorentzian function (solid line). The FWHM was determined on the fitted function. c) Confocal overview of the imaged region in a) before and after long-term RESOLFT time-lapse imaging. The RESOLFT timelapse recording (151.2 min of continuous recording) was performed in the indicated area $(23 \mu \mathrm{m} \times 24 \mu \mathrm{m})$. All images display raw data. Scale bars: a) upper images: 5 $\mu \mathrm{m}$, magnifications: $1 \mu \mathrm{m}, \mathrm{c}) 10 \mu \mathrm{m}$ (modified after [77]).

The confocal overview images show obvious bleaching in the recorded region after 151 minutes of continuous RESOLFT imaging within this position. Yet, the surrounding area shows labelled intact microtubule filaments and no sign of fragmented microtubules or signs of apoptosis. 


\subsubsection{In vivo RESOLFT-microscopy of intact larva}

As presented in Section 2.1.5, the light intensities used in RESOLFT imaging seem not to influence the cell viability within the time scale of imaging. Together with the capability of imaging in a thick and highly fluorescent tissue, RESOLFT-microscopy should be able to facilitate high-resolution imaging in living animals. To analyse the possibility of in vivo RESOLFT-microscopy, RESOLFT imaging was performed using an intact second instar larva as a sample. The larva was placed within a custom built cavity (Section 4.2) filled with Schneider cell culture medium, which restrained the overall movement of the larva during imaging (Figure $2.10 \mathrm{a}$ ). However, irregular contraction of the body wall muscles was not restricted.

RESOLFT imaging was performed by focussing trough the intact larval cuticle into the first cell layer. The larval cuticle is relatively transparent, but upon focussing through the cuticle, the excitation light can be absorbed or deteriorated by scattering. As described in Section 2.1.2, the rsEGFP2- $\alpha$-tubulin construct is expressed in nearly all cells types, highlighting the microtubule cytoskeleton virtually in all cells of the larva. To fully activate all rsEGFP2 molecules located in the first cell layer underneath the larval cuticle, a strong $405 \mathrm{~nm}$ light pulse was needed (20 $\mu$ s of $11 \mu \mathrm{W})$. This represents 4 times the light intensity which was used for RESOLFT imaging of isolated tissues (Figure 2.3). The deactivation pulse was set to $520 \mu \mathrm{s}$ with $32 \mu \mathrm{W}$ to obtain the best possible resolution, these parameters enabled highresolution RESOLFT imaging within the first cell layer underneath the cuticle of intact larva (Figure $2.10 \mathrm{c}$ ). The line profiles of the indicated positions demonstrate an achieved resolution of $\sim 50 \mathrm{~nm}$ in the RESOLFT image of intact larva, which enables a separation of co-aligned microtubules. This is not possible in the corresponding diffraction-limited confocal image (Figure $2.10 \mathrm{~d}$ ).

The irregular movements of the larval muscles impeded the recording of multiple RESOLFT frames of the same position. Still, single RESOLFT recordings of the microtubule network in living intact larva was feasible.

To underline the robustness of RESOLFT-microscopy for in vivo imaging of Drosophila melanogaster, additional examples of different positions within the larva are given in Figure 2.11. The visual resolution enhancement enables a much more detailed study of the dense microtubule network as in the corresponding confocal recordings. To further analyse the attained resolution enhancement of the RESOLFT imaging of the whole intact larva, the average FWHM of the rsEGFP2$\alpha$-tubulin filaments within an in vivo-RESOLFT image was assessed. Line profiles averaged across ten pixel sizes were taken at several positions and the FWHM was determined (Figure 2.12). 
a

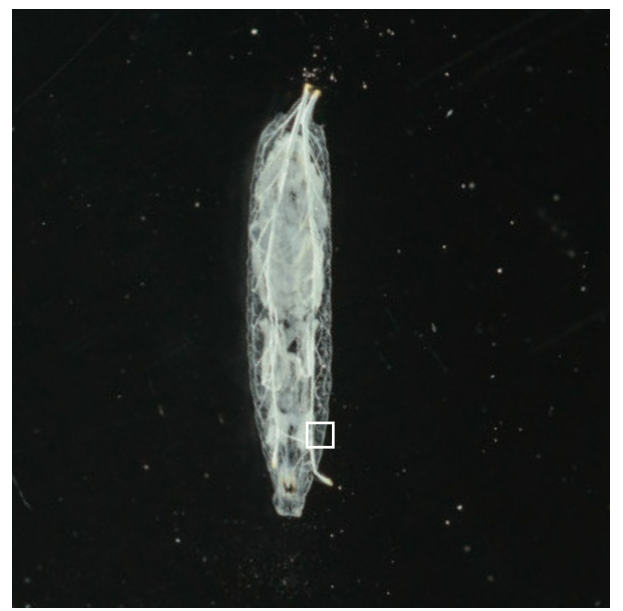

C
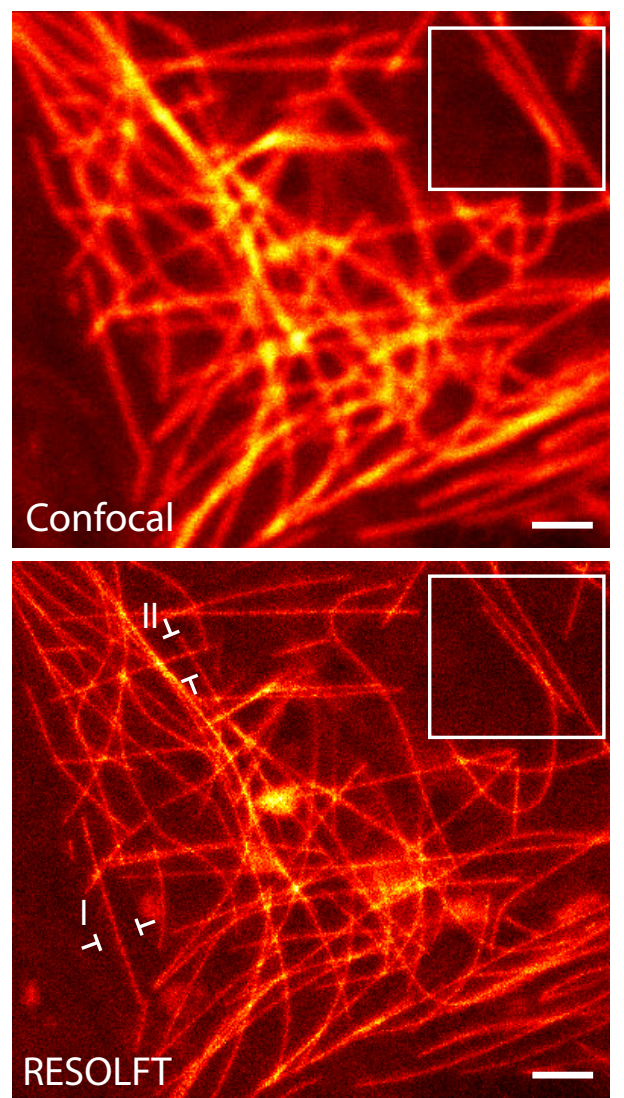
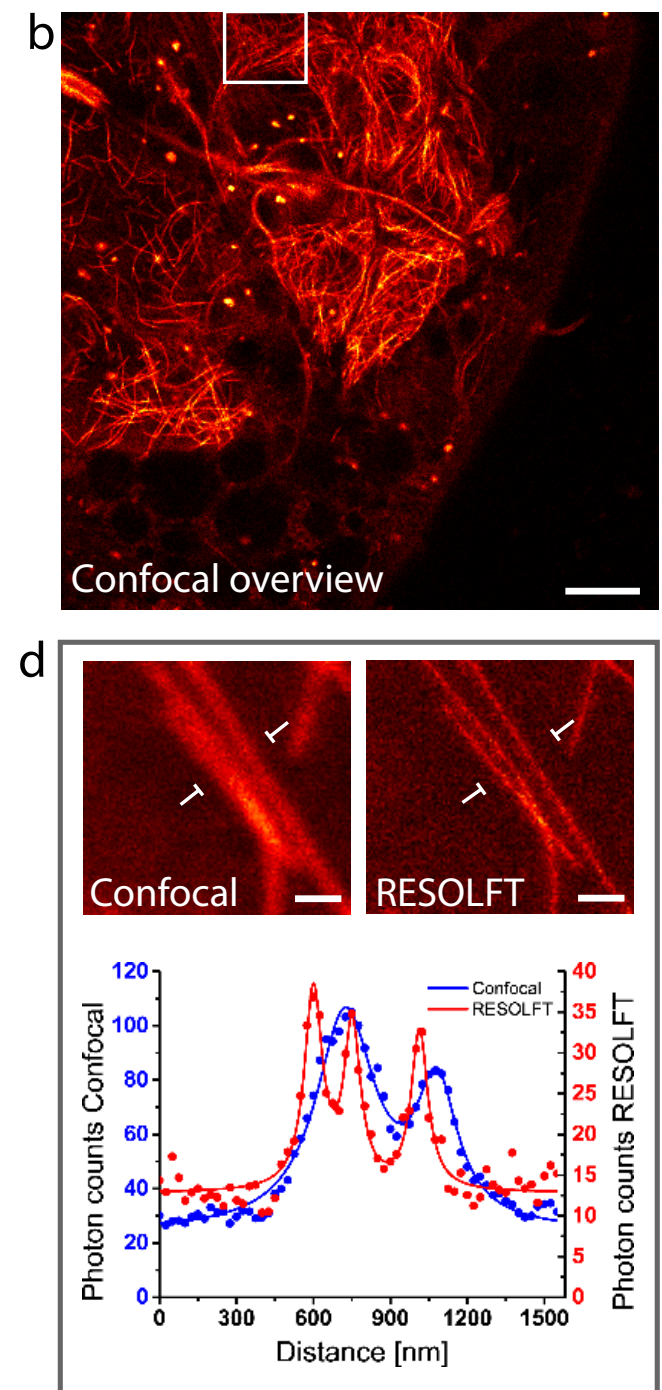

e

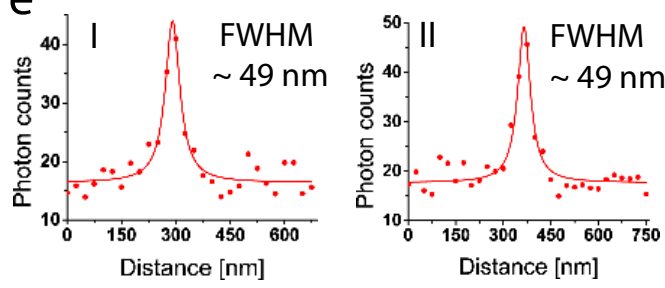

Figure 2.10: In vivo RESOLFT-microscopy of intact Drosophila melanogaster larva. a) Living second instar larva expressing rsEGFP2- $\alpha$-tubulin placed under a coverslip between two spacers in Schneider's medium. b) Confocal overview image, recorded through the cuticle of the larva. c) Confocal (top) and corresponding RESOLFT (bottom) image of the area indicated in b). d) Top: Magnifications of the areas indicated in c). Bottom: Line profiles taken at the indicated positions. e) Line profiles taken at the positions indicated in c). The data points represent an average of three adjacent $(25 \mathrm{~nm}$ distance) measurements. The averaged data were fitted with a Lorentzian function (solid line). The FWHM was determined on the fitted function. Images display raw data. Scale bars: $10 \mu \mathrm{m} \mathrm{b}$ ), $1 \mu \mathrm{m} \mathrm{c}$ ), and $500 \mathrm{~nm} \mathrm{~d}$ ) (modified after [77]). 

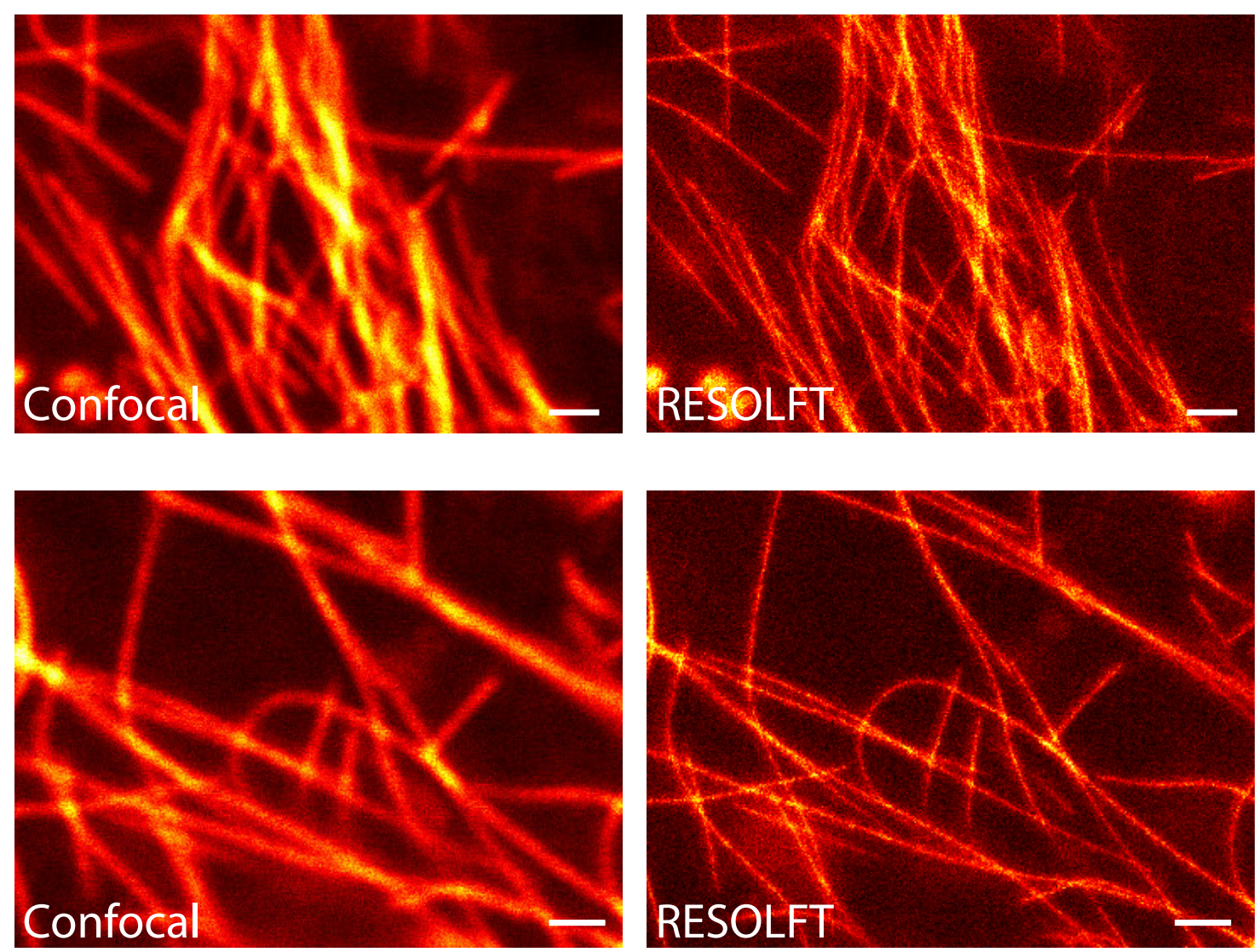

Figure 2.11: In vivo RESOLFT imaging of intact Drosophila melanogaster larva at different scan positions in the larva. Additional examples to Figure 2.10 of RESOLFT imaging taken on living larva by focussing through the intact cuticle of the larva at different positions. Confocal and corresponding RESOLFT images display raw data. Scale bars: $1 \mu \mathrm{m}$.

The mean value of the determined FWHMs in RESOLFT images recorded by focussing through the intact cuticle into the larva was $60 \mathrm{~nm}$ (Figure 2.12).

All displayed RESOLFT images of Drosophila melanogaster verify the applicability of RESOLFT imaging for live-cell high-resolution imaging in this multicellular organism. Further, it demonstrates the usability of RESOLFT imaging for in vivo imaging of intact organisms for the first time. The attained resolution in the RESOLFT imaging mode was robustly between $\sim 50 \mathrm{~nm}-60 \mathrm{~nm}$ (Figure 2.3, 2.12). The utilization of the switching capability of RSFP offers an additional improvement in imaging at high imaging depths. The low light intensities needed in RESOLFT imaging to achieve high-resolution enabled the recording of cellular dynamics with high frame rates (Figure 2.8) or over a long observation time (Figure 2.9). Moreover, the usability of RESOLFT imaging for imaging of three dimensional volumes with high-resolution along all optical axes was demonstrated. 


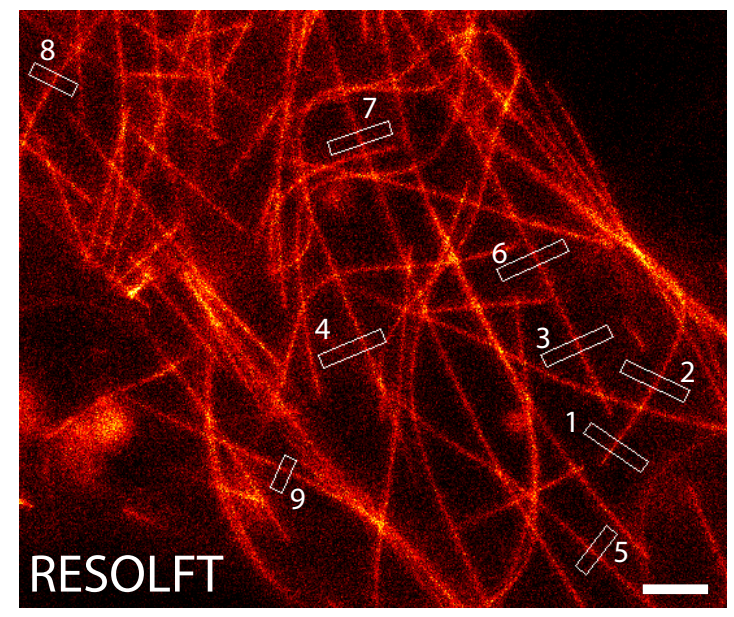

\begin{tabular}{|c|c|}
\hline Line Profile & FWHM [nm] \\
\hline 1 & 53.1 \\
\hline 2 & 54.2 \\
\hline 3 & 61.9 \\
\hline 4 & 63.2 \\
\hline 5 & 60.7 \\
\hline 6 & 58.2 \\
\hline 7 & 56.8 \\
\hline 8 & 66 \\
\hline 9 & 60.1 \\
\hline Average & $59.3 \pm 4 \mathrm{~nm}$ \\
\hline
\end{tabular}
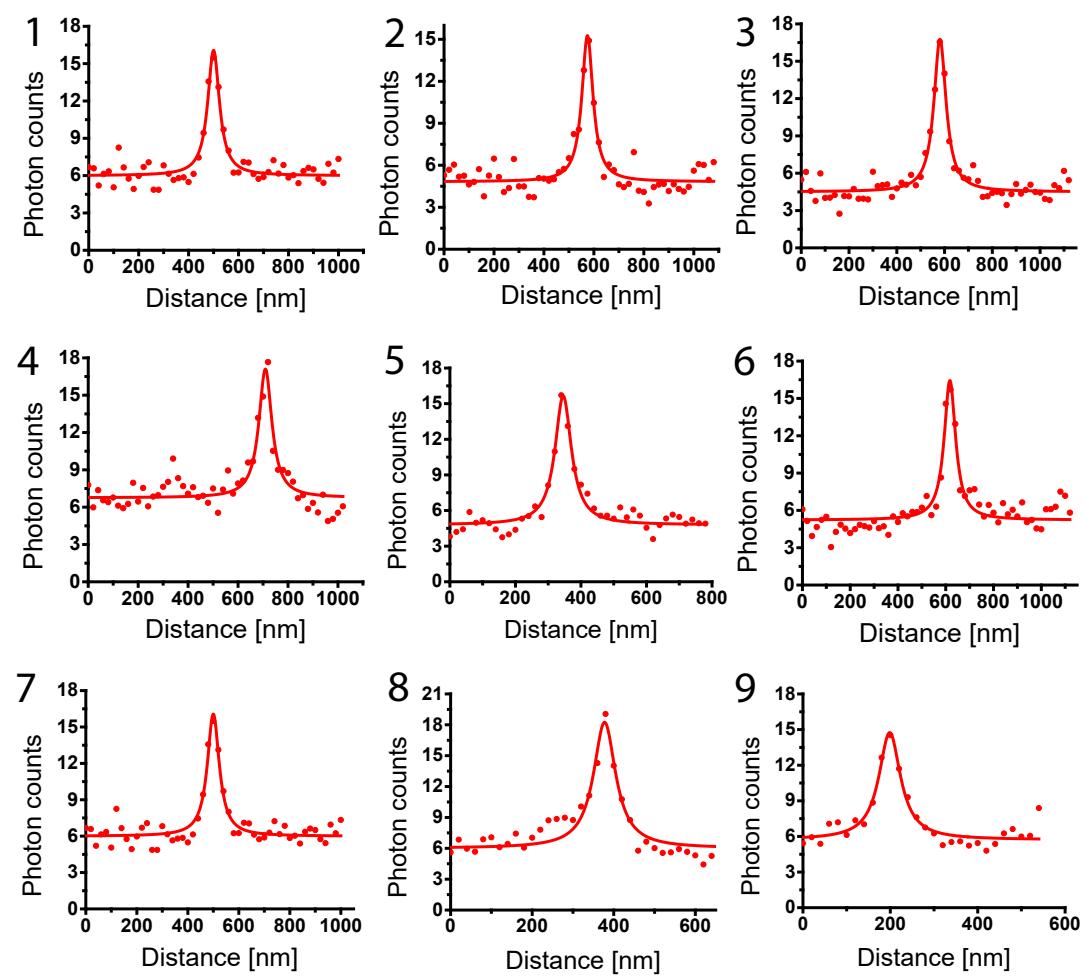

Figure 2.12: Determination of the achieved lateral image resolution in intact larvae. RESOLFT recording taken on an intact living second instar larva expressing rsEGFP2- $\alpha$-tubulin. The data points represent an average of ten adjacent measurements (20 nm distance between each measurement). The averaged data were fitted with a Lorentzian function (solid line) and the FWHM was determined on the fitted function. Scale bar: $1 \mu \mathrm{m}$ (modified after [77]). 


\subsection{High-resolution RESOLFT-microscopy of Arabidopsis thaliana}

High-resolution RESOLFT-microscopy requires low light intensities of $\sim 1-40$ $k W / \mathrm{cm}^{2}$ to achieve a sub-diffraction resolution image [45]. Hence RESOLFT imaging might be highly suitable for high resolution imaging in light sensitive specimens such as plant cells.

This section describes the establishment of RESOLFT imaging in Arabidopsis thaliana for the first time and evaluates its usability for high-resolution imaging within this model organism of plant science.

The generation of MAP4-rsEGFP2 expressing Arabidopsis thaliana was kindly performed by Dr. Hassan Ghareeb (University of Göttingen).

\subsubsection{Generation of rsEGFP2 expressing Arabidopsis thaliana}

To characterise the usability of RESOLFT-microscopy in high-resolution plant imaging, similar as described before (Section 2.1.1), microtubule filaments were chosen as the target structure for imaging. To label the microtubule network in Arabidopsis thaliana, a strategy reported by Marc et al. [80] was applied.

The expression of a fusion protein consisting of the microtubule associated protein (MAP) 4 and a fluorescent protein marks the microtubule network in the cell specifically by attachment of MAP4 to the microtubule filaments. To enable RESOLFTmicroscopy, the eGFP coding sequence was replaced by the sequence of rsEGFP2. The construct was expressed under control of the ubiquitin-10 promoter, which displays strong expression levels in various cell types [81]. The generated tranformants of Arabodopsis thaliana were crossed homozygous as individual stable lines. Plants of the T4 generation were used for imaging (Section 2.2.1).

All images in this Section display only raw data, except selected images where smoothing with a 1.2 pixel wide Gaussian was used to reduce the background noise in the RESOLFT images. All image modifications are denoted in the respective figures. Image manipulations like deconvolution or background subtraction were not performed.

\subsubsection{Characterisation of MAP4-rsEGFP2 expression pattern in Arabidopsis thaliana}

To characterise the expression of the MAP4-rsEGFP2 construct in the generated Arabidopsis thaliana transformants, various cell types were analysed using live cell 

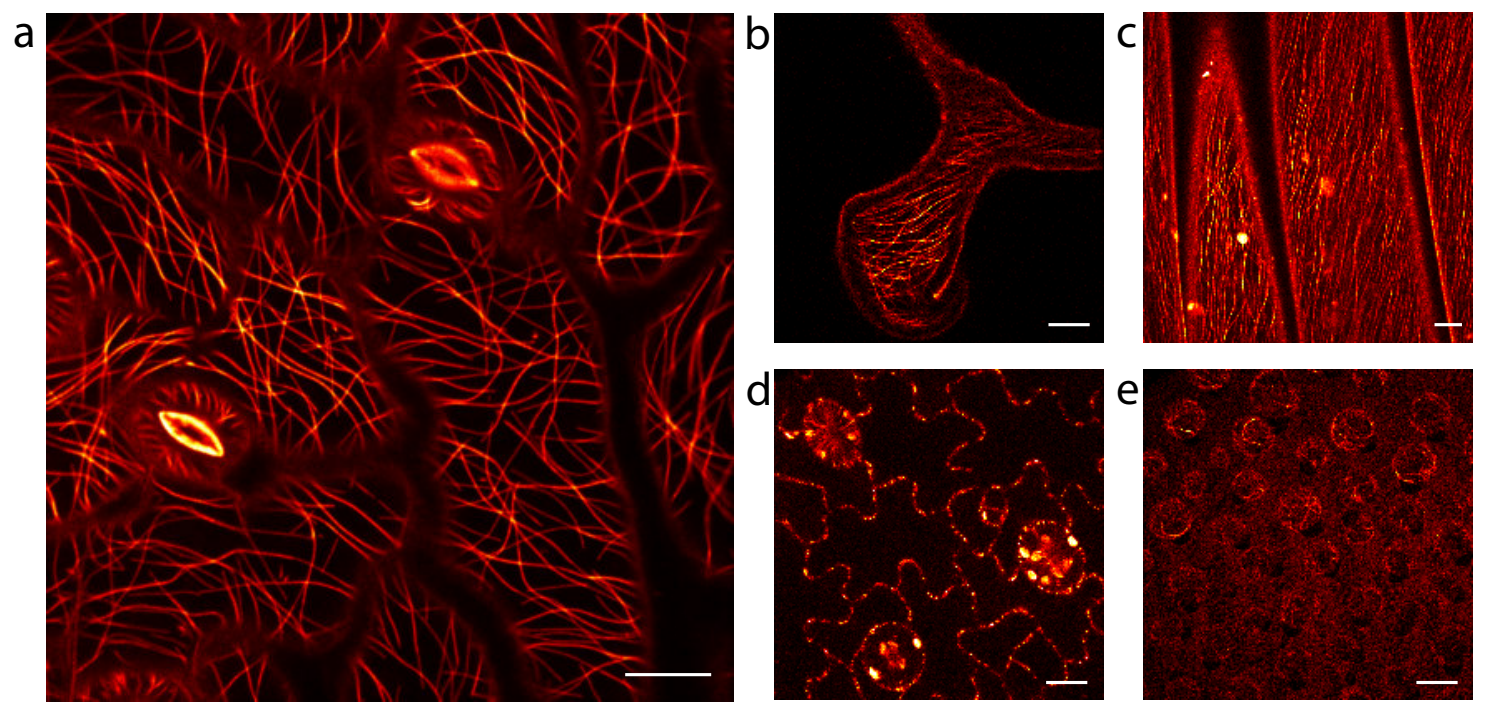

Figure 2.13: Live cell confocal overview images of MAP4-rsEGFP2 labelled microtubule filaments in different cell types of Arabidopsis thaliana expressing MAP4-rsEGFP2. a) upper leaf epidermis, b) trichome on the upper leaf epidermis, c) thin slice of petiole, d) lower leaf epidermis cells, e) sepals cells. Scale bars: $10 \mu \mathrm{m}$.

fluorescence microscopy. Labelling of the microtubule network was visible in leaf epidermis cells, stomata cells, trichome, cells of petiole, sepals and petals cells. The fluorescence recordings of the respective cell types are shown in Figure 2.13.

The confocal recordings of leaf epidermis cells and trichomes (Figure 2.13 a, and b) display a specific labelling of microtubule filaments in these cell types. Here the labelled microtubule network exhibits a good signal to noise ratio. Thin slices of petiole epidermis reveal an expression of the MAP4-rsEGFP2 construct and display a polarised orientation of microtubules in this cell type (Figure $2.13 \mathrm{c}$ ). The microtubule network in sepals show a weak labelling next to a strong background signal in the detection channel (Figure $2.13 \mathrm{e}$ ). The autofluorescence of chloroplast is visible in the GFP-detection channel (500 - $550 \mathrm{~nm})$ as round shaped organelles located underneath the microtubule network in various cell types (Figure $2.13 \mathrm{c}, \mathrm{d}$ ).

Altogether, the generated MAP4-rsEGFP2 tranformants showed an expression of the MAP4-rsEGFP2 construct in various cell types of Arabidopsis thaliana resulting in specific staining of the microtubule network. An expression or labelling of microtubule filaments in root cells was not detectable. 


\subsubsection{Autofluorescence suppression by fluorescence lifetime gating}

a
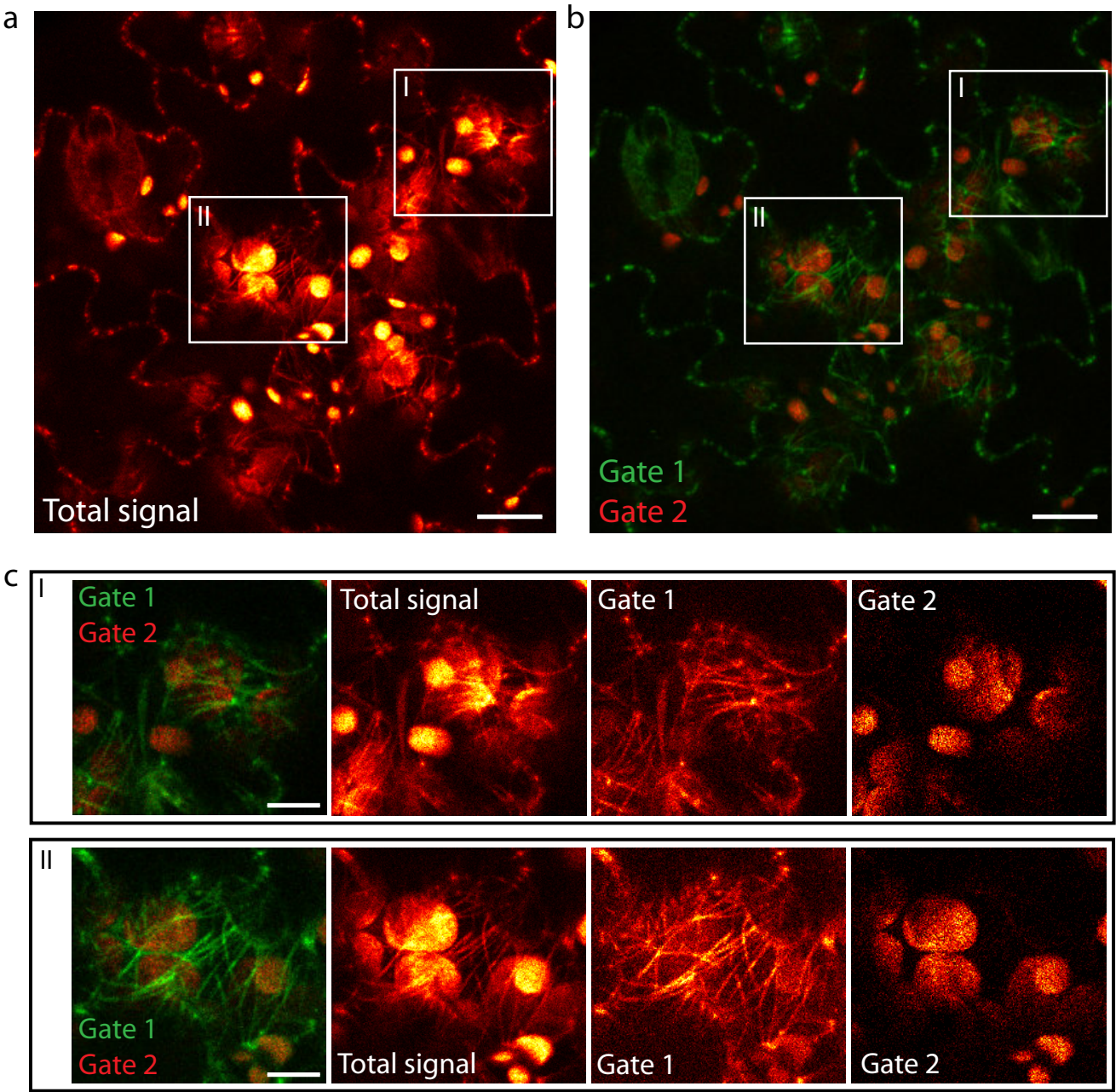

Figure 2.14: Autofluorescence reduction in live cell microscopy via fluorescence lifetime gating. a) non-gated confocal image of lower leaf epidermis cells illustrating autofluorescence of chloroplasts in the GFP-detection channel (detection filter: $500 \mathrm{~nm}-550 \mathrm{~nm}$ ). The fluorescence of the MAP4-rsEGFP2 construct is not discriminable from the autofluorescence of chloroplast b) false color coded confocal image of a) illustrating separation of GFP signal and chloroplast autofluorescence via fluorescence lifetime gating. c) raw data of single gated channels (Gate 1 and 2) and non gated (Total signal) image of highlighted areas in a) and b). Scale bars: a), b) $10 \mu \mathrm{m}$ and c) $5 \mu \mathrm{m}$.

The depicted images in Figure 2.13 were recorded using a continuous wave (cw) light source for excitation, i.e. a continuous amount of photons is emitted over the whole excitation time period. To analyse if the observed autofluorescence coming from the chloroplasts can be separated from the rsEGFP2 signal using fluorescence lifetime gating, the excitation laser was changed to a pulsed light source. A pulsed 
excitation laser emits a photon burst at a given frequency, e.g. every $25 \mathrm{~ns}$ (at 40 $\mathrm{MHz}$ repetition rate). The fluorescence detection of the microscope can be restricted to small time periods (e.g. $1 \mathrm{~ns}-2 \mathrm{~ns}$ ) which is defined with respect to the rsEGFP2 fluorescence lifetime of $\sim 1.6 \mathrm{~ns}$ [53]. Depending on the arrival time after the excitation pulse, the detected photons can be assigned to the GFP detection channel or to an additional detection channel.

The autofluorescence of chloroplast is mainly caused by chlorophylls which feature a relative short fluorescence lifetime of $\sim 250-300 \mathrm{ps}$ [82]. The chloroplast detection gate was set to 0-312.5 ps, while the rsEGFP2 detection channel was set to 2.656 $16.71 \mathrm{~ns}$. Although the main signal of rsEGFP2 was not detected, this combination of time gates showed the lowest crosstalk between the rsEGFP2 detection channel and the autofluorescence. The individual gates were false color coded to enable a visual separation. The rsEGFP2 gate is shown in green color (Gate 1) while the autofluorescence channel is in red (Gate 2) (Figure 2.14).

The fluorescence signal of the microtubule filaments labelled with the MAP4rsEGFP2 construct is overlapping with the autofluorescence of chloroplasts, which impedes the tracing of single filaments in the non-gated image (Figure $2.14 \mathrm{a}$ ). The gated rsEGFP2 detection channel (Gate 1) shows strong reduction in the autofluorescence and enables the tracking of filaments over the whole image. The autofluorescence gate (Gate 2) depicts the position of the chloroplast in the image (Figure $2.14 \mathrm{c}$ ). The false color coding strengthened the visual separation of the two channels. In the gated detection channel (Gate 1) the filaments are easily traceable over single chloroplasts, while in the non-gated detection channel (total signal), the filaments are overlaid by the background fluorescence.

Using fluorescence lifetime gating, it is therefore possible to separate the autofluorescence emanating from the chloroplast specifically from the fluorescence signal of the MAP4-rsEGFP2 labelled microtubule filaments (Figure 2.14). Thus the rsEGFP2 labelled structure can be studied even if the fluorescence is overlapping with the autofluorescence of the chloroplasts.

\subsubsection{RESOLFT-microscopy of Arabidopsis thaliana}

To analyse the applicability of RESOLFT-microscopy for live cell high-resolution imaging in Arabidopsis thaliana, small pieces ( $4 \mathrm{~mm}$ x $4 \mathrm{~mm}$ ) of green leaves were mounted in PBS-buffer and used as a sample for RESOLFT imaging (Section 4.9). To record RESOLFT-images within upper leaf epidermis cells, imaging parameters had to be identified for this specific cell type. For activation of the rsEGFP2 molecules, a strong activation pulse of $405 \mathrm{~nm}$ at $11 \mu \mathrm{W}$ for $20 \mu$ s was needed. For 

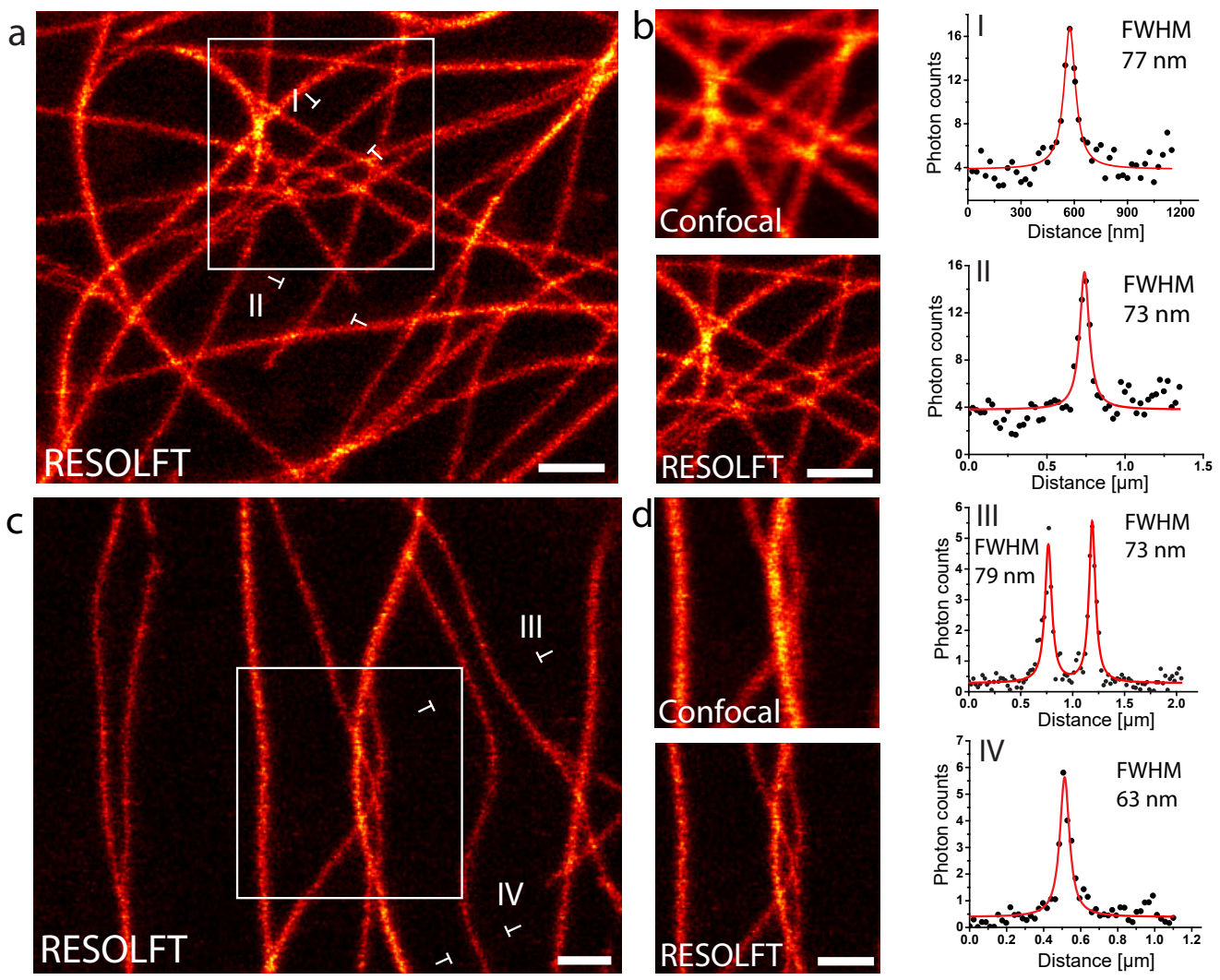

Figure 2.15: High-resolution RESOLFT imaging of MAP4-rsEGFP2 labelled tubulin filaments in leaf epidermis cells. a) RESOLFT image of MAP4rsEGFP2 labelled microtubule filaments. b) confocal and corresponding RESOLFT image of highlighted area in a). c) RESOLFT image of MAP4-rsEGFP2 labelled tubulin filaments in leaf epidermis cells. Line profiles were taken at the indicated positions in a) and c). The data points represent an average of five adjacent measurements (at $22 \mathrm{~nm}$ (a) or $23 \mathrm{~nm}$ (c) distance each). The averaged data were fitted with a Lorentzian function (solid line). The FWHM was determined on the fitted function. Scale bars: $1 \mu \mathrm{m}$.

deactivation of molecules in the periphery the off-switching was performed using the $488 \mathrm{~nm}$ off-switching doughnut-shaped beam for $480 \mu \mathrm{s}$ with $32 \mu \mathrm{W}$. The fluorescence of the residual rsEGFP2 molecules in the fluorescent on-state at the very center of the excitation volume was subsequently read out for $40 \mu \mathrm{s}$ using a $488 \mathrm{~nm}$ Gaussian shaped readout pulse with $14.4 \mu \mathrm{W}$. To record RESOLFT images, these three steps of illumination were performed at each scanning position in the image, i.e. at every pixel of $25 \mathrm{~nm}$ width. To increase the signal to noise ratio, every line was scanned twice and the signal accumulated.

To validate the achieved resolution enhancement, a regular diffraction-limited confocal image was recorded next to the RESOLFT image, by applying the same imaging parameters except performing the off-switching illumination step. 
The recorded RESOLFT images of upper epidermis leaf cells are depicted in Figure 2.15 .

As seen before, the MAP4-rsEGFP2 construct shows a uniformly labelling of the microtubule network within leaf epidermis cells. The degree of labelling is sufficient to highlight the microtubule filaments in the high-resolution RESOLFT images (Figure 2.15). In epidermis cells, the microtubule filaments feature sites of crossings and interconnections as well as co-alignment (Figure $2.15 \mathrm{~b}$ ). In the RESOLFT mode, filaments which were located close together can be optically separated which is not possible in the respective confocal counterpart image. As shown in the line profiles (Figure 2.15), FWHMs across single filaments of $\sim 60 \mathrm{~nm}-70 \mathrm{~nm}$ were achieved in the RESOLFT imaging mode. This represents a resolution enhancement of $3-4$ times compared to the best possible diffraction-limited confocal image. Further, the total photon counts given in the line profile point out the low background signal in leaf epidermis cells of only 0 - 4 counts in average.

\subsubsection{STED-microscopy of MAP4-rsEGFP2 labelled microtubule filaments in leaf epidermis cells}
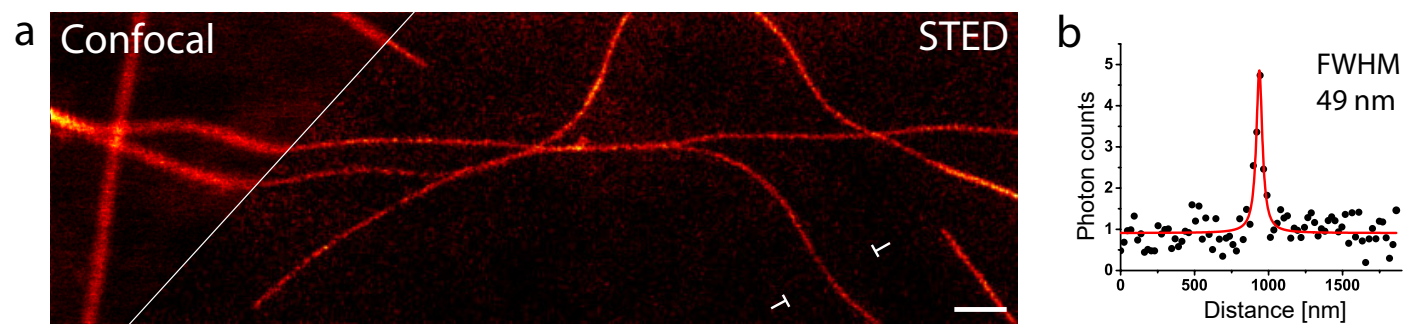

Figure 2.16: High-resolution STED imaging of MAP4-rsEGFP2 labelled microtubule filaments. a) Confocal and STED image of MAP4-rsEGFP2 labelled microtuble filaments in upper epidermis leaf cell. b) Line profile taken at the indicated position in a). The data points represent an average of five adjacent measurements at $23 \mathrm{~nm}$ distance each). The averaged data were fitted using a Lorentzian function (solid line). The FWHM was determined on the fitted function. Scale bar: $1 \mu \mathrm{m}$.

So far, only one study has reported on the application of STED imaging in plant cells [75], albeit only root epidermis cells were used which do not feature numerous light absorbing molecules compared to photosynthesis active cells [76].

To analyse whether STED-microscopy is applicable for high-resolution imaging in living leaf epidermis cells, STED imaging was performed on MAP4-rsEGFP2 labelled microtubule filaments in upper leaf epidermis cells. To record STED images 


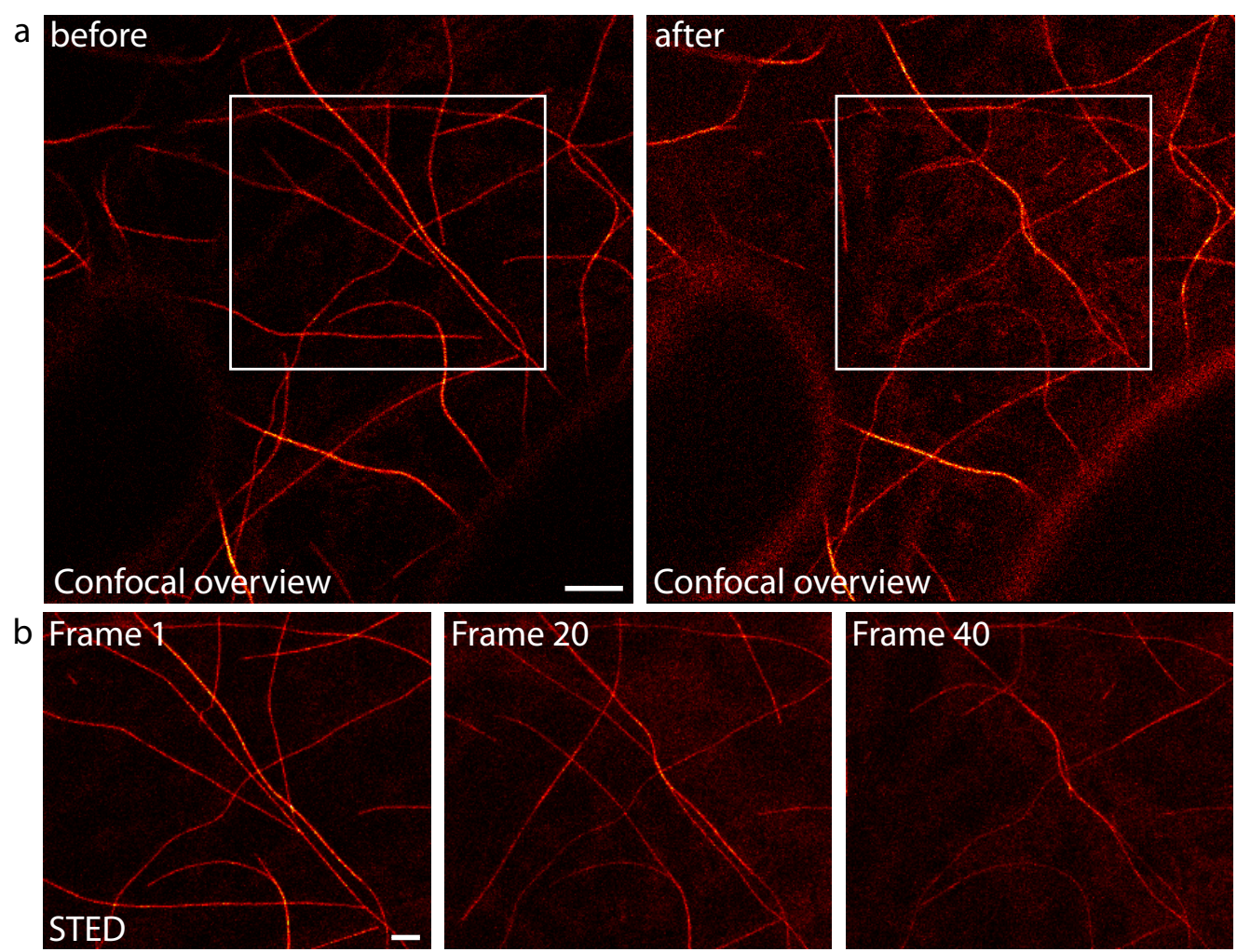

Figure 2.17: High-resolution STED time-lapse imaging of MAP4rsEGFP2 labelled tubulin filaments in leaf epidermis cells. a) Confocal overview images recorded before and after recording of 40 STED frames in the indicated area. b) Three selected out of a total of 40 STED frames illustrating dynamics of MAP4-rsEGFP2 labelled microtubules of the area indicated in a) while imaging. Imaged were recorded continuously. Recording time per image: 31 seconds. Images display raw data smoothed using a 1.2 pixel wide Gaussian function. Scale bars: a) $5 \mu \mathrm{m}, \mathrm{b}) 2 \mu \mathrm{m}$.

using rsEPGF2 as a fluorescent label, the following identified imaging parameters were applied: first, activation of rsEGFP2 with light of $405 \mathrm{~nm}$ for $20 \mu$ s using 1.1 $\mu \mathrm{W}$. Next, the molecules located at the periphery of the excitation volume were switched to the fluorescent off-state via stimulated emission (STED-laser $587 \mathrm{~nm}$, $7.86 \mathrm{~mW}, 20 \mathrm{MHz}$ repetition rate), while the molecules located at the very center were read out using light of $488 \mathrm{~nm}$ for $30 \mu \mathrm{s}$ using $18.7 \mu \mathrm{W}$. Every line was scanned three times and the signal was accumulated to achieve the final image. The identified imaging parameters, enabled to record single STED images in leaf epidermis cells (Figure 2.16). In order to quantify the attained resolution in the STED image, a line profile measured across a single filament averaged over five pixel distance was analysed and demonstrated a FWHM of $49 \mathrm{~nm}$.

To examine the usability of STED-microscopy for time-lapse recordings in leaf epidermis cells, all imaging parameters where kept constant in respect to Figure 2.16 
except that the STED intensity was decreased to $3.6 \mathrm{~mW}$. These parameters enabled continuous recording of 40 STED frames of an area of $\sim 642 \mu \mathrm{m}^{2}$ (Figure 2.17).

During the image acquisition, the microtubule network showed strong modifications while recording continuously 40 STED frames, filaments located inside the observation area displayed a decreased dynamic growth and apparent depolymerization. Confocal overview recordings of the observation area before and after the long-term STED imaging, display the decrease in the number of filaments and the increase in the background signal in the recorded area (Figure 2.17 a).

Comparing the depicted selected frames in Figure $2.17 \mathrm{~b}$, a significant reduction in signal intensity of the MAP4-rsEGFP2 construct while recording the 40 STED frames becomes apparent. However, the fluorescence signal is still sufficient enough to highlight the microtubule filaments, especially in the last frame even at the decreasing signal to noise ratio.

It can be concluded that STED imaging in leaf epidermis cells of Arabidopsis thaliana is feasible to record single high-resolution images of microtubule filaments with $\sim 50$ $\mathrm{nm}$ resolution. Nevertheless, potential manipulations of the target structures may occur due to the high light intensities used in STED-microscopy within the time scale of image acquisition.

\subsubsection{RESOLFT time-lapse imaging of Arabidopsis thaliana}

During the acquisition of single RESOLFT frames (Section 2.2.4), no obvious signs of cellular distress were visible. The microtubule network in upper epidermis leaf cells exhibited a dynamic behaviour of growing as well as shortening of individual filaments. To analyse whether long-term RESOLFT recordings causes the same photo-manipulation of the microtubule network as STED-microscopy, 40 RESOLFT frames were recorded continuously. To capture fast dynamic changes of the microtubule network with high spatial resolution, the RESOLFT imaging parameters identified before (Figure 2.15) had to be adapted to reduce the acquisition time per single RESOLFT-frame. To record the movement of microtubule filaments at high-resolution within an area of $12.9 \mu \mathrm{m} \times 9.72 \mu \mathrm{m}$, the pixel size was set to 30 $\mathrm{nm}$ to fully display the before achieved resolution of $\sim 75 \mathrm{~nm}$ (Figure 2.15). The off-switching time of the $488 \mathrm{~nm}$ off-switching doughnut-shaped laser beam ( $43 \mu \mathrm{W})$ was shortened to $120 \mu \mathrm{s}$, while no line accumulation was applied. Beside this, the activation was shorten to $12 \mu \mathrm{s}$ at $3.5 \mu \mathrm{W}$ and the readout was performed with $30 \mu \mathrm{s}$ at $15.8 \mu \mathrm{W}$. These parameters resulted in an acquisition time of $\sim 22$ seconds for a single RESOLFT frame. 

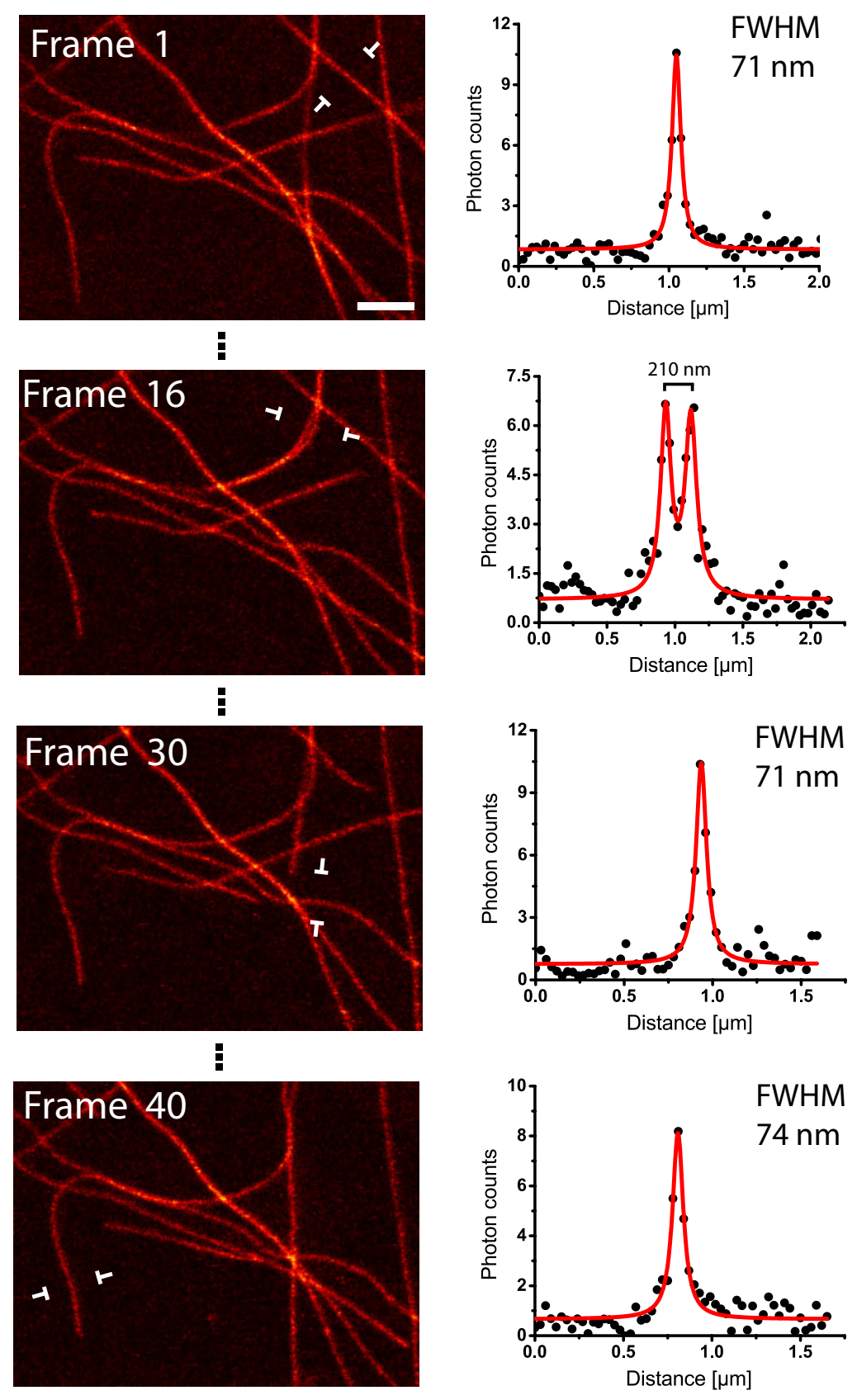

Figure 2.18: Time-lapse RESOLFT imaging of MAP4-rsEGFP2 labelled microtubule filaments in upper leaf epidermis cells. Four selected from in total 40 continuously recorded RESOLFT frames, illustrating the dynamics of the MAP4-rsEGFP2 labelled microtubule network in upper leaf epidermis cells. The line profiles of indicated positions represent an average of five adjacent measurements (of $30 \mathrm{~nm}$ distance each) across single filaments at the indicated positions. The averaged data were fitted with a Lorentzian function (solid line). The FWHM was determined on the fitted function. Images display raw data smooth using a 1.2 pixel wide Gaussian function. Scale bar: $2 \mu \mathrm{m}$. 
Applying these RESOLFT parameters, the dynamic behaviour of the microtubule network in upper leaf epidermis cells could be captured at high spatial resolution in 40 consecutive RESOLFT frames (Figure 2.18). The selected frames from the recorded time-lapse series illustrate the dynamic changes of the microtubule network in upper leaf epidermis cells. The reduction in the off-switching time by $\sim$ 4 fold could be successfully compensated by a strong increase in the intensity of the off-swichting beam. This is illustrated in the line profiles taken across single filaments (averaged along five pixel positions) reporting a resolution of $\sim 75 \mathrm{~nm}$ in the recorded time-lapse series, indicating sufficient off-switching.

The colormap used in Figure 2.18 is normalised to the first frame of the recorded time-lapse series. As can be seen by comparing the first and the last frame, the fluorescence signal of the individual microtubule filaments is not reduced significantly over the 40 frames. This absence of bleaching is in contrast to the noticeable bleaching in the recorded 40 RESOLFT time-lapse series taken in Drosophila melanogaster (Figure 2.8). The absence of bleaching is further strengthened by the fact that the resolution is not declining over the recorded time-lapse series as can be seen in the depicted line profiles (Figure 2.18).

\subsubsection{Bleaching analysis of rsEGFP2 in Arabidopsis thaliana}

The bleaching analysis on long term RESOLFT recordings of MAP4-rsEGFP2labelled microtubule filaments presented in this section was carried out together with Dr. Lars Frahm.

\subsubsection{Bleaching analysis}

To analyse the bleaching behaviour of the MAP4-rsEPGF2 labelled microtubules in RESOLFT recordings, an area of $47 \mu \mathrm{m}^{2}$ was imaged continuously over 250 frames in the RESOLFT mode. The following imaging parameters were applied: $405 \mathrm{~nm}$ activation: $20 \mu \mathrm{s}, 3.8 \mu \mathrm{W}, 488 \mathrm{~nm}$ off-switching: $340 \mu \mathrm{s}, 11.5 \mu \mathrm{W}, 488 \mathrm{~nm}$ readout: $40 \mu \mathrm{s} 23.5 \mu \mathrm{W}$. These parameters resulted in a recording time of $\sim 12$ seconds per RESOLFT frame.

As observed before, the microtubule filaments in upper leaf epidermis cells exhibited a dynamic behaviour; in the recorded time-lapse series, the total number of filaments in the observed area is constantly changing since filaments are moving in and out of the observation area.

The fast dynamics of the microtubule network impeded a bleaching analysis based on the average fluorescence signal per frame, since the total number of filaments is constantly changing over the recorded time-lapse series (Figure 2.19 a). 

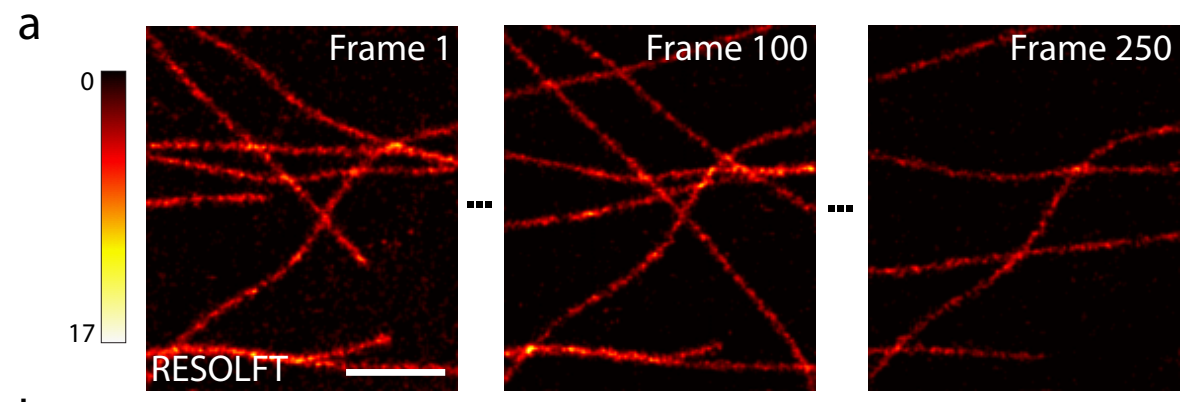

b
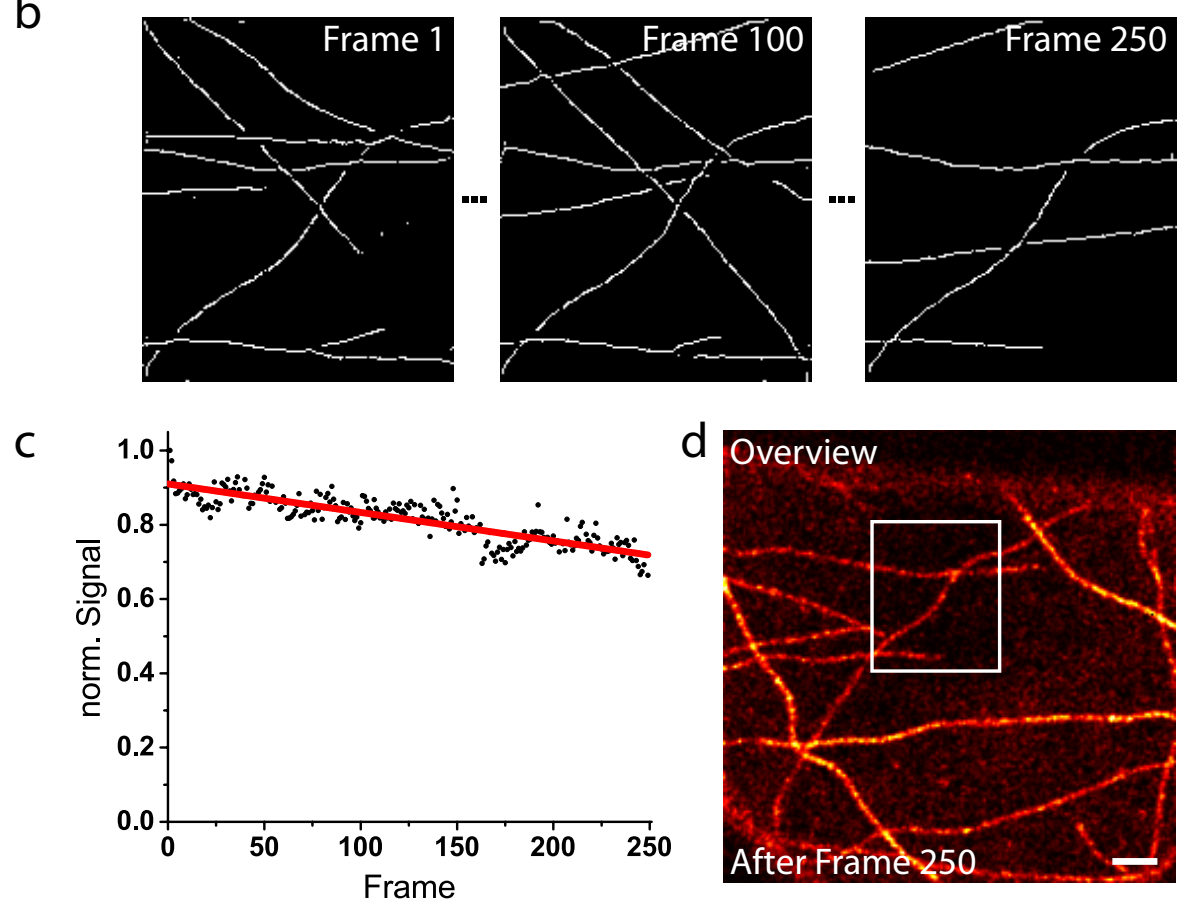

Figure 2.19: Bleaching analysis of MAP4-rsEGFP2 in RESOLFT timelapse recordings of leaf epidermis cells. a) Three selected from in total 250 RESOLFT frames illustrating the bleaching behaviour of MAP4-rsEGFP2 labelled microtubules in the RESOLFT mode. The color map of the depicted images is normalized to the signal intensity of the first frame. b) Bleaching analysis of single RESOLFT frames. The average signal intensity of all shown white pixels per frame were analysed. Pixels corresponding to the background noise were rejected for the analysis. c) Bleaching curve of average signal per filament in each RESOLFT frame. Data (black dots) was fitted with a linear function (red line). d) Confocal overview image after RESOLFT time-lapse imaging of 250 frames of the indicated position. Scale bars: $2 \mu \mathrm{m}$.

A change in the number of filaments in the observation area will also alter the average signal per frame; therefore a possible bleaching of the fluorescent label rsEGFP2 cannot be detected.

In order to analyse the bleaching of rsEGFP2 in the time-lapse recordings, an algorithm was applied which determines the average fluorescence signal per filament in each frame. 
Following this, the bleaching analysis is independent of the total number of filaments in the frame (Figure $2.19 \mathrm{~b}$ ).

The applied algorithm detects filaments in each frame and calculates the average fluorescence signal of all pixels which were assigned to be part of the filaments (depicted in white lines in Figure 2.19 b). Figure 2.19 c) depicts the plotted average brightness per filament in each single frame of the recorded time-lapse RESOLFT series. Here a reduction of the average filament brightness of $\sim 25 \%$ over the recorded 250 frames is discernible.

\subsubsection{FRAP measurements on MAP4-rsEGFP2 labelled microtubule filaments}
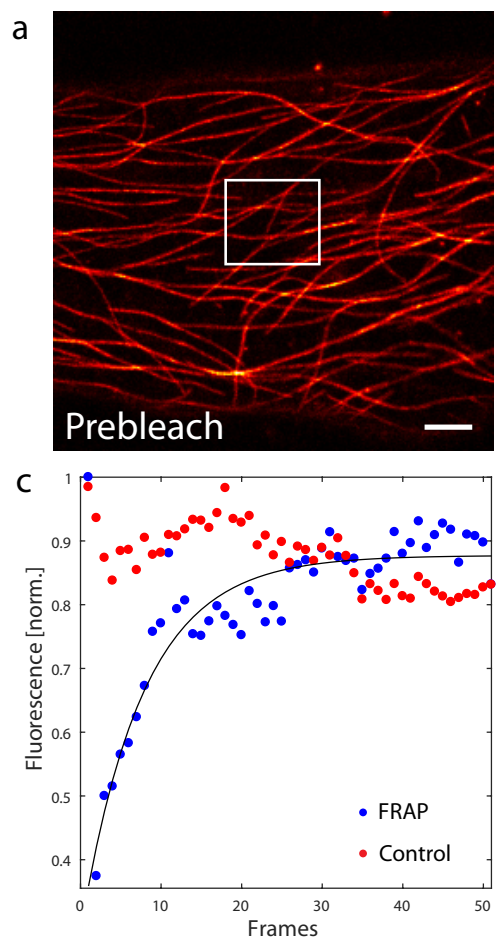
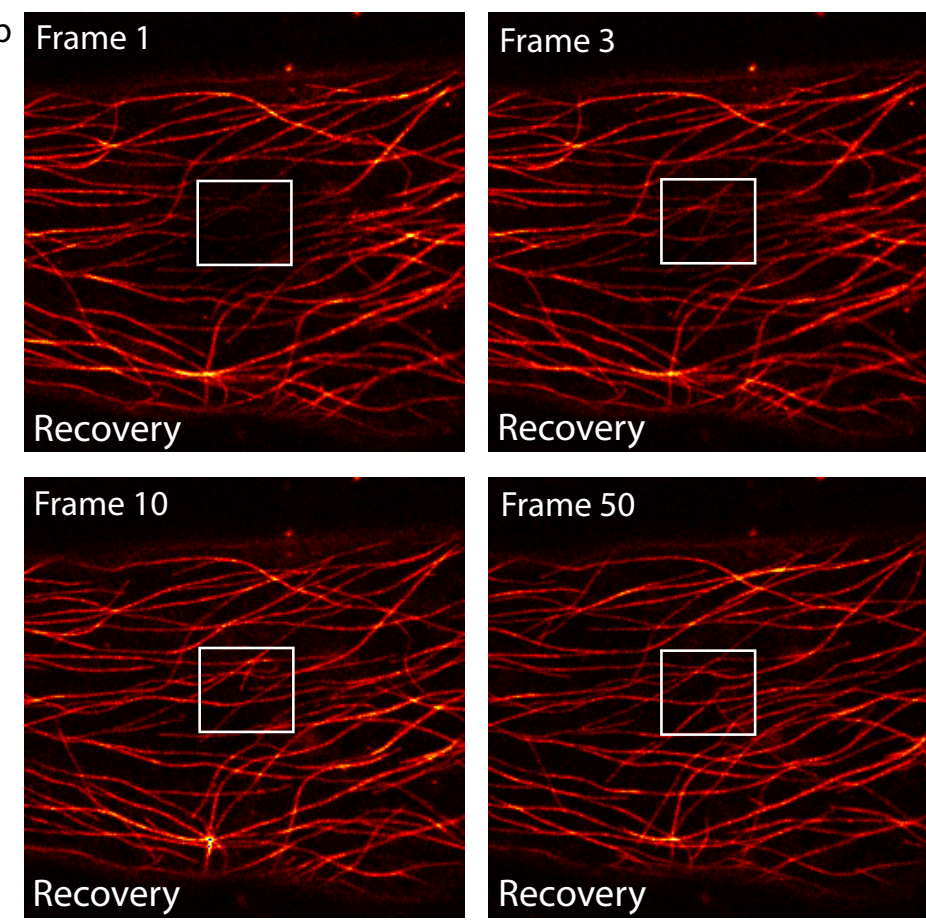

Figure 2.20: FRAP measurement on MAP4-rsEGFP2 labelled microtubules in upper leaf epidermis cells of Arabidopsis thaliana. a) Confocal overview image before bleaching of indicated positions. b) Four selected frames from in total 50 consecutive frames illustrating the recovery after photobleaching of indicated region. c) Analysis of FRAP measurement on indicated area in a) and b). Blue dots depict the average fluorescence signal in the indicated area in each frame of the recorded time-lapse series after photo-bleaching. Red dots depict the average fluorescence outside the indicated area per frame in the recorded time-lapse series. Black line depicts an exponential fit of the FRAP data points. Recovery to $50 \%$ after 5 frames. Recording time of each frame: 1.3 seconds. Scale bar: $5 \mu \mathrm{m}$. 
To examine whether the reduced bleaching rate of rsEGFP2 in the RESOLFT mode is the result of replacement of the bleached MAP4-rsEGFP2 molecules at the microtubule filaments, fluorescence recovery after photobleaching (FRAP) measurements were performed (Section 4.12).

In upper leaf epidermis cells a region of interest was illuminated with light of $405 \mathrm{~nm}$ and $488 \mathrm{nmm}$ simultaneously to bleach the MAP4-rsEGFP2 protein in this region (indicated region in Figure 2.20). Subsequently, 50 consecutive confocal overview frames were recorded to analyse whether the bleached fluorescence will recover. As a control, the average fluorescence in the non-bleached region was used. The analysis of the average fluorescence signal in the recorded confocal time-lapse series was restricted on the filaments in each frame, similar to as described in Section 2.2.7. The normalized average fluorescence of the control region is shown in red dots whereas the normalized average fluorescence of the bleached area is depicted in blue dots in Figure $2.20 \mathrm{c}$ ). After the bleaching pulse, the bleached region displays fast recovery of the average fluorescence signal. After $\sim 7$ seconds the fluorescence is recovered to $50 \%$ of its initial value and reaches $90 \%$ after $\sim 39$ seconds (Figure $2.20 \mathrm{c}$ ).

The performed FRAP experiment revealed a fast replacement of bleached MAP4rsEGFP2 proteins in upper leaf epidermis cells within seconds.

\subsubsection{Filament counting in RESOLFT time-lapse series}

The applied algorithm in section 2.2.7 was used to determine the average brightness of a single filament in every frame of the recorded time-lapse RESOLFT series. If the brightness of a single filaments is known, the filaments in each frame can be counted and tracked over time in a time-lapse series.

This is especially important if the optical resolution of the image is not sufficient to fully separate filaments located directly close together. In plant cells, microtubule filaments are often co-aligned over several micrometer distances and move along each other. The achieved resolution in RESOLFT imaging of plant cells of $\sim 70 \mathrm{~nm}$ represents an $\sim 3-4$ times resolution enhancement compared to regular diffraction-limited confocal imaging. However, it is still insufficient to optically separate filaments which are located directly next to each other.

This is well exemplified in the depicted frames of the recorded RESOLFT time-lapse series shown in Figure 2.21. In frame 128, two filaments are located closely to each other and cannot be optically separated (marked by the arrow in frame 128 Figure 2.21). The data analysis identified two filaments at this position via the fluorescence signal at this position which is represented by the green color coding (arrow in frame 128 Figure 2.21). 

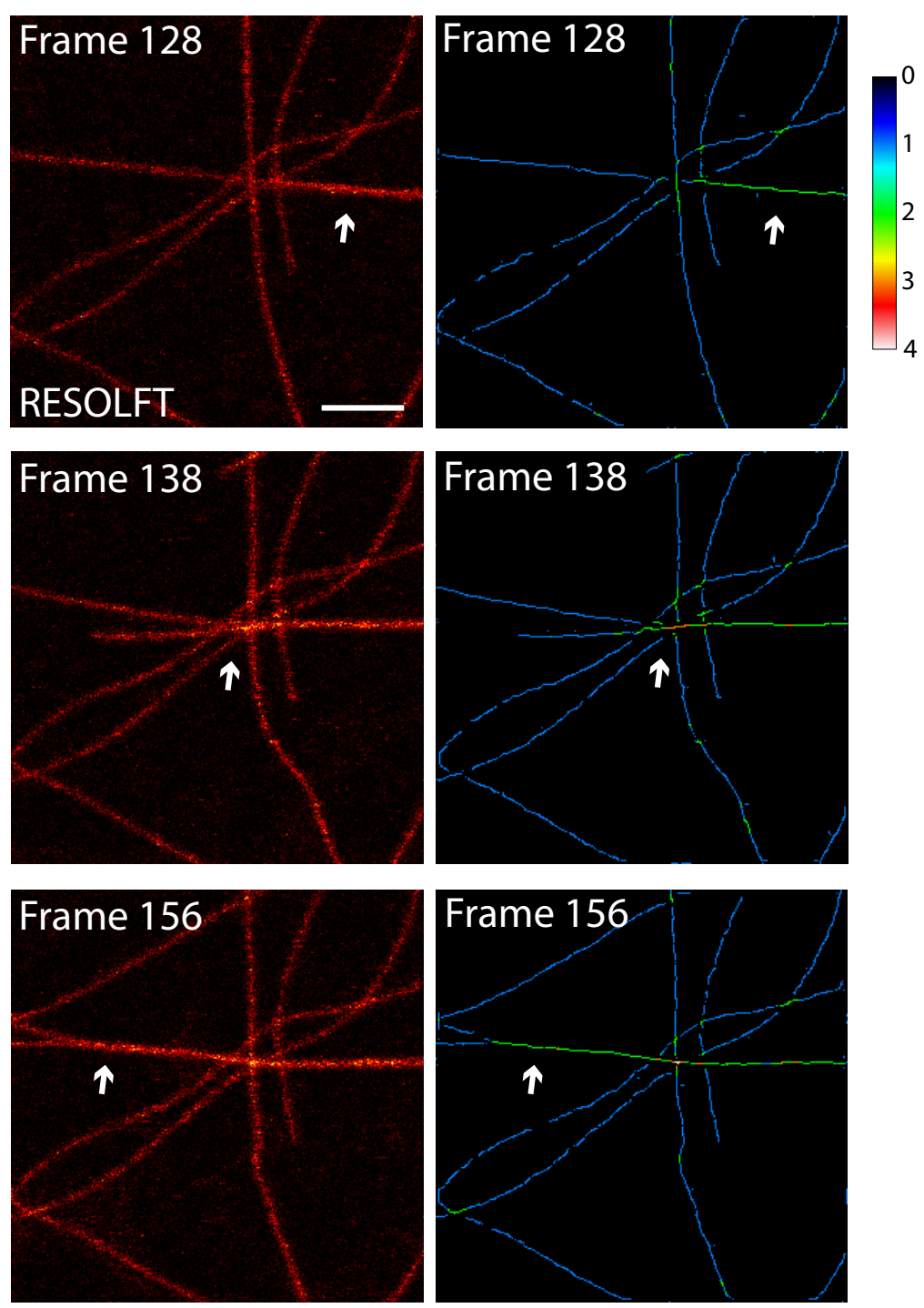

Figure 2.21: Filament counting and tracking in time-lapse RESOLFT recordings of the microtubule network in upper leaf epidermis cells of Arabidopsis thaliana. Left images: depicted frames in a total of 200 recorded RESOLFT frames. Right images: False color coded images, every pixel in the frame is color coded with respect to the multitude of the average brightness of a single filament. Colormap illustrating the average brightness of 0 - 4 filaments. Scale bar: $2 \mu \mathrm{m}$.

As can be seen in frames 138 and 156, the movement of single filaments resulting in fusion and co-alignment of two filaments can be visualized and tracked over time in the recorded time-lapse series (arrows in frames 138 and 156 in Figure 2.21).

Together, the displayed recordings of rsEGFP2-MAP4 labelled microtubule filaments in Arabidopsis thaliana demonstrate the applicability of RESOLFTmicroscopy for high-resolution imaging in plant cells. RESOLFT imaging facilitated single images as well as time-lapse recordings featuring $\sim 70 \mathrm{~nm}$ resolution of upper 
epidermis cells. The bleaching of rsEGFP2 was negligible while recording continuously 250 RESOLFT frames while no signs of phototoxicity were discernible during the image acquisition. 


\section{Discussion}

\subsection{RESOLFT-microscopy of Drosophila melanogaster}

Since the first proof of principle RESOLFT demonstration by Hofmann et al. [20], several studies demonstrated the application of high-resolution RESOLFTmicroscopy in different living specimens (Section 1.4.3).

With this work the usability of RESOLFT-microscopy for high-resolution imaging of multicellular organisms was analysed and established for the first time.

\subsection{1 rsEGFP2- $\alpha$-tubulin expressing Drosophila melanogaster}

For the establishment of high-resolution RESOLFT-microscopy in Drosophila melanogaster, microtubule filaments were chosen as the target structure for imaging. Microtubule filaments are rigid rods with a fixed diameter of $\sim 25 \mathrm{~nm}$, which is $\sim 8$ times smaller than the size of the diffraction-limited excitation spot [83]. Using a small structure of fixed size, the resolution enhancement in the RESOLFT imaging mode is comparable along different experiments.

The stable genomic integration of the expression construct led to a sample with invariable and constant labelling of the target structure in all cells of Drosophila melanogaster. This enabled a comparison of the achieved resolution enhancement between various experiments and different configurations of the custom-built microscope used for imaging.

Previous reports on the application of RESOLFT-microscopy were based on tranfected single cells expressing RSFP fusion proteins to label a target structure in the cell (Section 1.4.3). Transfected cells exhibit variable expression levels of the fusion construct due to different numbers of expression plamids in the cells [84]. Further, overexpression of fusion proteins can cause numerous artefacts like protein mislocalization, erroneous morphology of the target structure and many others [59], [63].

For the establishment of RESOLFT-microscopy in Drosophila melanogaster, the expression level of the RSFP fusion construct was chosen to avoid overexpression artefacts so as not to affect the fitness of Drosophila melanogaster. Albeit, the fluorescent signal had to be sufficiently high to enable RESOLFT-microscopy of various cell types. As shown in Section 2.1.2, the additional expression of rsEGFP2$\alpha$-tubulin next to the endogenous protein did not interfere with the fitness of the generated Drosophila melanogaster stock. The labelling of microtubules during the oogenesis and spermatogenesis suggests no negatively impact of the rsEGFP2- $\alpha$ - 
tubulin expression on the embryogenesis and underlines the versatility of rsEGFP2 as a genetically encoded label for tagging of target proteins.

The Western blot analysis revealed a 3:1 ratio of non-labelled to rsEGFP2-labelled $\alpha$-tubulin protein in total cell lysate of third instar larvae (Section 2.1.2). This ratio can be seen as an average proportion for all tissues; small variations between tissues may exist, since it is possible that differences in gene expression and protein turnover lead to varied protein levels of the rsEGFP2-labelled $\alpha$-tubulin fusion protein. Albeit, the average fluorescence brightness of single rsEGFP2 labelled $\alpha$-tubulin filaments was similar in different types of tissues used for RESOLFT-microscopy in Section 2.1.

The fluorescence signal of single microtubule filaments was sufficient to enable RESOLFT-microscopy, it was however relatively low. The maximum fluorescence intensity of single tubulin filaments was in the range of $10-20$ photon counts in the RESOLFT mode featuring a $30-40 \mu$ s readout step. To increase the fluorescence signal of the labelled microtubule filaments, the degree of labelling could be enhanced. This could be achieved by using endogenous labelling techniques like the CRISPR/Cas9 system [85], [86], [87]. Endogenously labelling of the genomic loci of the $\alpha$-tubulin gene could enable a complete labelling of all $\alpha$-tubulin proteins in the cell. This would increase the fluorescence signal of the labelled tubulin filaments compared to the generated fly stock, where approximately one out of four $\alpha$-tubulin proteins is labelled by rsEGFP2.

\subsubsection{Optical resolution in RESOLFT imaging of Drosophila melanogaster}

As demonstrated in Section 2.1, RESOLFT-microscopy could be performed on isolated tissues as well as in intact larvae of Drosophila melanogaster. The achieved optical resolution in the RESOLFT mode was robustly in the range of $50 \mathrm{~nm}-60$ $\mathrm{nm}$ (Section 2.1.2), this represents a 4 - 5 fold enhancement compared to regular diffraction-limited confocal microscopy using a GFP derivative as a fluorescent label. The achieved resolution is in accordance with previously reported FWHMs of 45 - $48 \mathrm{~nm}$ of rsEGFP2-labelled Keratin filaments of single mammalian cells in RESOLFT-microscopy [45].

The achieved resolution in the RESOLFT recordings in this work is comparable to that of live-cell STED-microscopy using fluorescent proteins as label, which is typically in the range of $50 \mathrm{~nm}$ [88]. One advantage of STED-microscopy is that the off-switching of fluorescent molecules in the periphery of the excitation volume is in the ns time scale, whereas in RESOLFT it is in the $\mu$ s or ms timescale. Thus 
STED-microscopy is better suited for imaging of dynamic structures or processes. However, in STED-microscopy the applied light intensities are up to $\sim 10^{6}$ times higher compared to RSFP-based RESOLFT-microscopy, therefore RESOLFT is better suited for long-term imaging of living cells [46], [45].

To exclude an erroneous determination of the achieved resolution in RESOLFT recordings of Drosophila melanogaster, all line profiles in this work were taken along 3 - 10 pixel widths and the FWHM was determined by using a fitted function. This analysis is in contrast to some previously reported RESOLFT studies, where line profiles were taken along single pixels or in deconvolved images, resulting in erroneous small resolution values [56], [59], [89].

As can be seen in Figure 2.12, the determination of the FWHMs based on line profiles can show variations in the range of $\sim 10 \%$ if multiple line profiles were taken in the same image. This can be explained by variations in the local background signal at different positions in one image. Therefore in this work, in all RESOLFT images, line profiles were taken at multiple positions to determine the average resolution in each image (Figures 2.3 and 2.12).

The achieved optical resolution of $\sim 50 \mathrm{~nm}$ in RESOLFT recordings of Drosophila melanogaster could still be further improved. One major constraint to achieve better resolution was the limitation in the fluorescence signal in the RESOLFT mode. As demonstrated in Section 2.1.2, single microtubule filaments often exhibited only 10 - 20 photons as maximum intensity in RESOLFT recordings, even if a line accumulation of two iterations was applied.

To further increase the optical resolution in RESOLFT recordings, the off-switching doughnut shaped beam has to be applied with higher light intensities. Thus the total number of molecules resting in their fluorescent on-state at the center of the excitation volume will be further restricted to a smaller size, resulting in better optical resolution in the image. However, this will lead to a decrease in the signal to noise ratio, since the number of off-switched molecules in the excitation spot increases compared to the number of molecules remaining in the fluorescent on-state. Consequently, the residual fluorescence signal of the off-switched population in the periphery of the excitation spot contributes stronger to the image. Therefore it is important that the RSFP used features a very low residual fluorescence in the off-state. The RSFP rsEGFP2 exhibits a residual fluorescence in the off-state of $\sim 6-7 \%$, whereas rsEGFP has a residual fluorescence of $1-2 \%$ and could be used to achieve higher optical resolution in RESOLFT recordings of Drosophila melanogaster [45], [46]. However, the folding of rsEGFP at $37^{\circ} \mathrm{C}$ is inefficient, leading to low fluorescence in the target structure since many fluorescent proteins are not functionally 
folded [46], [55]. The folding efficiency of fluorescent proteins is in general better at lower temperatures, thus rsEGFP might be used in Drosophila melanogaster at room temperature.

Nevertheless, the off-switching of rsEGFP is in the range of $10-20 \mathrm{~ms}$, which is $\sim 25$ times slower compared to rsEGFP2, thus imaging of fast dynmics of the microtubule network may not be possible [45].

The limitation of the fluorescence signal in the RESOLFT recordings is further based on the utilization of a negative switching RSFP, since it is switching to the fluorescent off-state while the fluorescence is read out. Therefore the total number of photons per read out step is limited. This can be compensated by performing line accumulations with the drawback of longer acquisition times.

The signal limitation in RESOLFT-microscopy could be overcome by utilizing the RSFP Dreiklang. In Dreiklang, the on- and off-switching is decoupled from the read out of fluorescence. Hence the molecules restricted to a sub-diffraction size at the very center of the excitation spot can be read out until sufficient photons are detected. However, Dreiklang possesses a strong switching fatigue; after $\sim 160$ switching cycles, $50 \%$ of the Dreiklang molecules are bleached. Additionally, the off-switching of Dreiklang is in the timescale of ms which makes RESOLFT imaging of fast dynamic processes in living cells challenging [42].

Next to optimisation of the image acquisition, the resolution of the recorded RESOLFT images can be improved using deconvolution algorithms, as already illustrated in several previous RESOLFT studies [45], [56], [46], [89]. However all data shown in this work represents only raw data to fully report on the possibilities as well as the limitations of RESOLFT-microscopy in Drosophila melanogaster.

\subsubsection{Photostress in RESOLFT-microscopy}

The light intensities applied in RESOLFT recordings of isolated tissues of Drosophila melanogaster were in the range $5-40 \mathrm{~kW} / \mathrm{cm}^{2}$. This is in accordance with previously reported values of RESOLFT-microscopy using rsEGFP2 in single mammalian cells [45]. These intensities are comparable to those applied in scanning confocal microscopy $\left(10-50 \mathrm{~kW} / \mathrm{cm}^{2}\right)$ resulting in a total light dose of approximately 500 Joule $/ \mathrm{cm}^{2}$ per image [60], [90]. However, these light intensities are higher than what is sometimes regarded as a critical light intensity threshold in long-term imaging of developmental processes of only few Joule/ $\mathrm{cm}^{2}$ [60], [90].

Photostress is dependent on the wavelength of the applied excitation light [60], [91]. Illumination with light of $405 \mathrm{~nm}$ wavelength at $0.2 \mathrm{~kW} / \mathrm{cm}^{2}$ for 240 seconds or a total illumination dose of $48 \mathrm{Joule} / \mathrm{cm}^{2}$ can cause photodamage like photo-fixation 
and an impairment of cell division upon irradiation. If the same total light dose is applied using light of $514 \mathrm{~nm}$ wavelength, no similar cellular destructions occur [60]. Compared to the stochastic targeted nanoscopy methods, point scanning RESOLFT-microscopy restricts the damaging excitation light to small parts of the sample while the rest of the sample is not illuminated. Thus possible phototoxic effects could be better compensated by the cell since the excitation light is applied to a restricted area for a very short time period. In contrast, in the stochastic targeted method PALM, the whole cell is illuminated for e.g. 240 seconds using light intensities of $0.05 \mathrm{~kW} / \mathrm{cm}^{2}$ of $405 \mathrm{~nm}$ light and $0.2 \mathrm{~kW} / \mathrm{cm}^{2}$ of $488 \mathrm{~nm}$ light to capture 12000 frames [60].

In this work, the illumination with light of $405 \mathrm{~nm}$ was needed to activate the rsEGFP2 molecules at each scanning position. The average light dose applied per single RESOLFT frame was in the range of $2-10 \mathrm{Joule} / \mathrm{cm}^{2}$ in accordance with Grotjohann et al. [45]. Following this, in the long-term RESOLFT recordings of 40 RESOLFT frames (Figures 2.8 and 2.9), the critical $405 \mathrm{~nm}$ illumination dose of 48 Joule $/ \mathrm{cm}^{2}$ identified by Wäldchen et al., was exceeded [60]. However, in the performed RESOLFT recordings, no visual signs of photostress were noticed.

One way to decrease the photostress in RESOLFT imaging using rsEGFP2 might be the use of two photon illumination for activation of the off-switched rsEGFP2 molecules, thus avoiding the illumination with light of $405 \mathrm{~nm}$ wavelength. This can be achieved via a two photon process using a femtosecond-pulsed laser at $780 \mathrm{~nm}$ wavelength as demonstrated by Böhm et al. [92].

The photostress in RESOLFT imaging could be decreased further by using an RSFP which exhibits absorbance and emission spectra in the red spectral range. Light of longer wavelength is less damaging to cells, since it is less absorbed by cellular compounds [60]. This would be an additional advantage for tissue imaging, because UV-light is strongly absorbed and scattered by tissue at high imaging depths. As seen in Section 2.1.2, the UV-light intensities could be decreased 3 fold if imaging was performed in the first cell layer instead of imaging at $\sim 10 \mu \mathrm{m}$ distance to the cover slip inside the tissue.

\subsubsection{Tissue RESOLFT-microscopy}

Previous studies of RESOLFT-microscopy performed on tissues were based on imaging of single neurons in hippocampal brain slices. In these reports, single neurons were transduced to express an RSFP-Lifeact fusion protein. Lifeact is a short peptide of 17 amino acids which binds to F-actin filaments and thus highlights the actin network of the cell [93]. RESOLFT imaging was performed on neuronal spine necks 
and a FWHM of $\sim 70 \mathrm{~nm}-80 \mathrm{~nm}$ of these sub structures was reported. Actin fibers within the neurons were not visible [46], [89].

The indirect labelling of the F-actin network via transiently binding of Lifeact-RSFP fusion proteins allows for the replacement of bleached Lifeact-RSFP proteins and thus can compensates a decrease in the fluorescence signal during image acquisition. Overexpression of Lifeact-RSFP fusion proteins might lead to a volume staining of the neuronal spine neck, caused by mostly unbound Lifeact-RSFP proteins situated in the cytoplasm, resulting in a strong fluorescence signal.

This labelling of the F-actin network is in contrast to the labelling strategy applied in this work for visualisation of a filamentous target structure in tissues. In Section 2.1 direct labelling of the tubulin network was achieved via expression of a RSFP- $\alpha$-tubulin fusion construct. The rsEGFP2- $\alpha$-tubulin protein was integrated into microtubule filaments, therefore it cannot be easily replaced and is prone to bleaching during imaging. Further, the fluorescence signal of labelled single tubulin filaments which feature a diameter of $\sim 25 \mathrm{~nm}$, is lower compared to a semi-volume staining of the neuronal spine neck featuring a diameter of $70 \mathrm{~nm}-80 \mathrm{~nm}$ [83], [94]. However, as can be seen in Section 2.7, RESOLFT-microscopy can be used to record high-resolution images of single filaments up to $\sim 40 \mu \mathrm{m}$ deep inside living tissues. RESOLFT-microscopy features an additional improvement for imaging at higher imaging depths. As mentioned before, the switching capability of RSPFs led to an enhancement of the signal to noise ratio in the recorded RESOLFT images (Figure 2.7). This effect is based on the targeted off-switching of fluorescent proteins in the periphery of the focal spot, leading to a reduced background signal in the image [78], [79].

At increasing imaging depths, the optical resolution of the RESOLFT recordings decreased, most likely caused by aberrations of the excitation light in the tissue. A further study could assess whether two photon RESOLFT imaging can be performed to enable RESOLFT imaging with rsEGFP2 at higher imaging depths. As mentioned before, the activation of rsEGFP2 can be performed via a two photon process [92], however, it needs to be analysed if also the off-switching and readout of rsEGFP2 can be performed as two photon processes. In this case, the applied laser wavelengths will be strongly red shifted compared to the wavelength used in this study and thus will be less scattered and absorbed by the tissue. This might enable RESOLFT imaging with rsEGFP2 at imaging depths $>40 \mu \mathrm{m}$ in tissues.

So far there is no demonstration of a stochastic-targeted method (STORM, PALM) applied to living tissues. These methods are likely unsuited for imaging of thick specimens due to scattering and aberrations of the excitation light by the tissue. 
Further, in most stochastic-targeted methods the detection is based on a camera recording a large field of view. In this design, the detection is not restricted to a single small optical section of the tissues, meaning also out-of focus fluorescence is detected which results in a high background signal in the image.

\subsubsection{In vivo RESOLFT-microscopy}

With this work, the application of RESOLFT-microscopy was extended to in vivo imaging of intact living Drosophila melanogaster larvae (Section 2.10). The utilisation of a confocal point scanning RESOLFT-microscope allowed imaging through the intact cuticle of the living larva; however, irregular movement of the larva impeded the recording of multiple RESOLFT frames of the same position. The larva was mounted between two spacers in Schneider media and sealed with a coverslip. Due to absence of sedation, irregular muscle contractions of the larva occured during the image acquisition. Further work is needed to establish long-term RESOLFT imaging of Drosophila melanogaster including improved sample preparation. The larva could be placed in a specialized imaging chamber to sedate the larva and thus suppress muscle contractions while imaging. It had been shown previously that specialized sample preparations allow live cell recordings of living Drosophila larvae over long time periods including feeding and ventilation of the larva [95], [96]. Further, this would enable long-term in vivo RESOLFT imaging to study developmental processes over time with high optical resolution. As demonstrated in this work, using rsEGFP2 as a fluorescent probe time-lapse RESOLFT recordings of 40 consecutive frames in isolated tissues are possible (Figure 2.8). RESOLFT imaging with more than 40 frames will be complicated by bleaching of the fluorophores during the acquisition. However, the imaging period could be extended by performing regular diffraction-limited confocal time-lapse imaging first to reduce the bleaching of the rsEGFP2 proteins, with the possibility to switch to high-resolution RESOLFT imaging instantly, capturing the process of interest in high spatial resolution.

Next to optimization in the sample preparation, RSFPs in the red spectral region might be beneficial for in vivo RESOLFT imaging. Longer wavelengths are less absorbed and scattered by the cuticle of the larva and thus would enable RESOLFT imaging at high imaging depths. Further, the photostress induced by the excitation light would be reduced, since light of longer wavelengths features lower energy and is less absorbed by the tissue [60].

In the last years, the palette of fluorescent proteins was extended to the Near Infrared (NIR)-spectrum by engineering of e.g. bacterial phytochromes. These 
proteins are well suited for in vivo imaging since the absorbtion of hemoglobin, water and melanin is minimal in the NIR spectral reagion (650 nm to $900 \mathrm{~nm}$ ) [97]. So far no RSFP based on a bacterial phytochrome has been reported. However, few studies refer to the utilization of NIR-fluorescent proteins in Drosophila for diffraction limited confocal imaging. In 2014, Yu et al. reported on the utilization of the fluoresent protein IFP 2.0, which was engineered from a bacterial phytochrome that incorporates biliverdin as an external chromophore and features excitation and emission maxima at $690 \mathrm{~nm}$ and $711 \mathrm{~nm}$, respectively. It was successfully used for imaging of neurons in living Drosophila larvae [98]. In 2015, Yu et al. reported on the development of mIFP as an improved monomeric bacterial phytochrome which was used for labelling of several cellular structures of Drosophila [99]. However, in all demonstrations, the additional expression of a heme oxygenase was necessary to increase the amount of free biliverdin in the cells since the endogenous levels of biliverdin are low in Drosophila cells resulting in weak fluorescence signal in tissues [98], [99].

\subsubsection{Conclusions and Outlook}

This work presents the characterisation and establishment of RESOLFT-microscopy in Drosophila melanogaster. As it could been shown for the first time, RESOLFTmicroscopy is capable of high-resolution tissue and in vivo imaging of Drosophila featuring a lateral resolution of 50 - $60 \mathrm{~nm}$. The used fluorescent label rsEGFP2 demonstrated its versatility as a genetically encoded tag for high-resolution RESOLFT imaging in a multicellular organism without influencing the fitness of the living sample.

Based on the presented establishment of RESOLFT-microscopy in Drosophila, RESOLFT-microscopy could be used for high resolution live cell imaging in the field of cell biology or developmental biology of Drosophila. Therefore, structures or proteins of interest should be labelled with the GFP derivative rsEGFP2, enabling a characterisation of their localization and dynamics on the nanoscale.

One possible application of RESOLFT-microscopy would be the characterisation of mitochondrial protein localization since mitochondria feature a diameter of $\sim$ $200 \mathrm{~nm}$, which is below the optical resolution limit of conventional light microscopes. Therefore, high resolution imaging is needed to study the sub-mitochondrial protein localization.

Since the development of rsEGFP2, several other RSFPs have been reported (Section 1.4.3). Further studies are needed to fully compare these RSFPs and their 
performance for high-resolution RESOLFT imaging in Drosophila. With ongoing development of RSFPs, the palette will be expanded to RSFPs in the NIR-spectral region, which might be beneficial for tissue and in vivo imaging. Thus, their utilization would reduce the possible phototoxic effects in living systems and allow imaging deeper inside tissues or whole animals.

Next to the improvement by parallelization of the off-switching step of negative switching RSFPs by Chymrov et al. [50], the point scanning RESOLFT-microscope approach should be improved to achieve faster RESOLFT imaging. One way would be the establishment of adaptive scanning. Hence scan positions where no structures are located could be skipped and the recording time of a single RESOLFT frame could be reduced. This would further reduce the photostress during imaging since many illumination steps might be omitted.

Based on the described establishment of RESOLFT-microscopy in Drosophila, the applicability of RESOLFT-microscopy in other model organisms like Caenorhabditis elegans and Danio rerio should be evaluated and characterised in the future.

\subsection{RESOLFT-microscopy of Arabidopsis thaliana}

This work reports on the characterisation and establishment of high-resolution RESOLFT-microscopy of the model plant Arabidopsis thaliana for the first time. The low light intensities applied in RESOLFT-microscopy make it particularly suitable for imaging in light absorbing plant cells.

\subsubsection{High-resolution imaging of Arabidopsis thaliana}

Previous demonstrations of live cell nanoscopy in plant cells were limited to two studies (Section 1.6). Liesche et al. reported on STORM-microscopy with optical resolution of $\sim 100 \mathrm{~nm}$ and visualised cellulose bundles in living onion epidermis cells. The TIRF-STORM approach used in this study restricted the imaging to the apical cell wall of epidermis cells, since the excitation light decays exponentially from the coverslip-medium interface (100 - $200 \mathrm{~nm}$ along the axial axis) [74]. In 2011, Kleine-Vehn et al. reported on STED imaging of YFP-labelled PIN proteins in living non-photosynthetic root epidermis cells of Arabidopsis thaliana [75].

Next to these studies, there are many reports on the application of SIM-microscopy in plant cells (reviewed by [72]). As mentioned before, SIM is by definition not a nanoscopy method because it is still diffraction-limited. The optical resolution of SIM is limited to $\sim 120 \mathrm{~nm}$ laterally and $\sim 350 \mathrm{~nm}$ axially [64]. To achieve a better optical resolution, SIM has to be combined with nonlinear approaches, like saturated illumination (SSIM) or e.g. photoswitchable fluorescent proteins [27], [100]. 
However, there are no demonstrations of the application of these approaches in plant cells yet.

In the work of Komis et al., SIM-microscopy was used to analyse the dynamic changes of the microtubule network in hypocotyl cells of Arabidopsis [101]. Similar to as performed in Section 2.2, the microtubule filaments were labelled by the expression of a MAP4-GFP construct following Marc et al., [80]. The application of SIM-microscopy enabled the visualisation of single microtubule filaments featuring a FWHM of $135 \mathrm{~nm}$ using a 1.57 NA 100x objective.

Spherical aberrations caused by light scattering at the plant cell wall can lead to reconstruction artefacts in the post-processing of SIM data. To reduce these aberrations during the imaging, etiolation of the plants was performed in this study. Growing plants in complete darkness leads to a thinning of the outer epidermal cell wall, thus reducing spherical aberrations while imaging. Nevertheless, this is a strong sample manipulation, since it represents abnormal physiological conditions, which can alter the target structure. This sample manipulation is in contrast to the performed sample preparation in this work. For the RESOLFT recordings of Arabidopsis thaliana, seedlings were grown under physiological conditions with $16 \mathrm{~h}$ of light illumination (Section 4.7). From these plants, small pieces (4 mm x $4 \mathrm{~mm}$ ) of leaves were cut out and used as sample without any additional manipulations (See Section 5.3). The recorded images presented in Section 2.2 demonstrate that RESOLFT-microscopy can be used for high resolution imaging in plant cells without any sample manipulation.

\subsubsection{Labelling of microtubule network by MAP4-rsEGFP2 expression}

The labelling strategy of Marc et al. [80] was used to specifically label microtubule filaments by the attachment of a microtubule binding protein fused with a fluorescent protein. The MAP4-rsEGFP2 construct was expressed in leaf epidermis cells, stomata cells, trichomes, cells of petiole, sepals and petals cells and displayed uniform and specific labelling of microtubule filaments in these cell types.

An expression of the MAP4-rsEGFP2 fusion protein in root cells was not found. However, the ubiquitin-10 promoter used here is known to be active in root cells [81]. The absence of expression in root cells could be explained by the performed transformation via Agrobacterium tumefaciens. The integration of the expression construct occurs randomly into the genome. Accordingly, position effects can influence the expression pattern. An integration in genome regions which are transcriptionally silent in root cells, might suppress an expression in the generated transformants. 
The MAP4-rsEGFP2 expression level in leaf epidermis cells was sufficient for highresolution RESOLFT and STED imaging in this cell type. However, the maximum fluorescence intensity of single microtubule filaments was in the range of $5-15$ counts in the RESOLFT mode (Figure 2.15).

The MAP4-rsEGFP2 construct is expressed under control of the ubiquitin-10 promotor which exhibits a constant expression level and is well suited to be used in stable lines of transformed plants [81]. The Cauliflower Mosaic Virus (CaMV) 35S promoter features a stronger expression level than the used ubiquitin-10 promoter and could therefore be used to enhance the fluorescence signal of MAP4-rsEGFP2 for the RESOLFT recordings [81], [102]. This can be done using a transient transformation of single leaves using Agrobacterium tumefaciens. For the generation of stable transformants, this promotor was not used since it shows invariable expression levels and is sensitive for gene silencing, which in turn could result in weak expression levels in stable transformants [81], [103].

\subsubsection{Autofluorescence suppression by fluorescence lifetime gating}

In Section 2.14, fluorescence lifetime gating was used to suppress the autofluorescence from chloroplasts in the rsEGFP2 detection channel during confocal live cell recordings of leaf epidermis cells. The chloroplasts are one of the main sources of autofluorescene in the wavelength region of GFP in plant cells [104]. Chloroplasts contain various light absorbing and emitting molecules, like chlorophylls, xanthophylls and carotenes. Chlorophyll a has a relatively broad emission spectrum with a strong emission peak at $680 \mathrm{~nm}$ [82]. Illumination with blue light of $470 \mathrm{~nm}$ leads to the emission in the range of $480 \mathrm{~nm}-600 \mathrm{~nm}$, which is overlapping with the emission of rsEGFP2 and is thus detected in the GFP detection channel (500 nm $550 \mathrm{~nm})[105]$.

As demonstrated in Figure 2.14, the performed fluorescence lifetime gating resulted in a reduction of the chloroplast autofluorescence in the rsEGFP2 detection channel and thus enabled the analysis of rsEGFP2 labelled microtubule filaments which were located directly next to the chloroplasts.

In previous studies, sample modifications were used to reduce the autofluorescence of the chloroplasts in fluorescence imaging of plant cells. These modifications include the extraction of chlorophylls via ethanol or gene silencing to suppress the xanthophyll and chlorophyll production in leaves [104], [106]. Albeit, these modifications represent a strong modification of the plant and can alter the sample.

The performed fluorescence lifetime gating allowed the suppression of the chloro- 
plast autofluorescence without altering the physiology of the sample. Thus green plants which were grown under physiological conditions could be used for imaging without any further sample manipulation.

While working on this project, a similar approach was indepently reported by Kodama in 2016 [105]. In this study, fluorescence lifetime gating was used to reduce the autofluorescence of chloroplasts in Metrosideros polymorpha and Arabidopsis thaliana in conventional confocal microscopy.

It has been demonstrated previously that RESOLFT can be combined with fluorescence lifetime imaging to separate two spectrally similar RSFPs based on their respective fluorescence lifetimes [53]. However, fluorescence lifetime gating has not used in planta with high-resolution imaging to suppress autofluorescence so far. Further work is required to extend the RESOLFT imaging of MAP4-rsEGFP2 labelled microtubules to analyse whether the fluorescence lifetime imaging in planta can be used to reduce the autofluorescence of chloroplasts in high-resolution RESOLFT images.

\subsubsection{Live-cell STED-microscopy of Arabidopsis thaliana}

So far only one study reports on the application of high resolution STED-microscopy in living plant cells. In this study STED-microscopy was used to analyse the protein localization of YFP-labelled PIN2 auxin efflux carriers at the membrane of Arabidopsis thaliana root epidermis cells [75]. The study was restricted to single STED images of non-photosynthesis active root cells, time-lapse imaging was not performed.

The high light intensities used in STED-microscopy might cause photodamage in photosynthesis active plant cells. The red shifted STED light could be absorbed by chlorophylls, which could then lead to the production of reactive oxygen species and to overheating of the sample [76].

In this work, STED-microscopy was performed to analyse whether STEDmicroscopy is applicable for high-resolution imaging in photosynthetic active leaf epidermis cells. For STED-microscopy of rsEGFP2, a pulsed STED-laser at $587 \mathrm{~nm}$ with intensities of 3-7 $\mathrm{mW}$ (measured at the back focal plane of the objective lens) was used.

In the recorded STED time-lapse series, a modification of the microtubule network was discernible during the image acquisition of 40 continuously recorded STED frames (Figure 2.17). The filaments showed reduced growth and apparent depolymerization. This modification of the target structure was not visible if single STED frames were recorded (Figure 2.2.5). The observed changes in the microtubule net- 
work within the STED time-lapse imaging is in contrast to the recorded time-lapse series of 40 consecutive RESOLFT frames. Here no visual signs of photoinduced changes of the microtubule network were visible (Figure 2.18).

The absence of photodamage during the time-lapse RESOLFT recordings might be explained by the different light intensities applied in STED- and RESOLFTmicroscopy. As mentioned before, the light intensities used in RESOLFT-microscopy are up to several orders of magnitude lower compared to those in STED-microscopy [45], hence photodamage is less likely in the RESOLFT imaging.

\subsubsection{Time-lapse RESOLFT recordings}

The development of fast switching RSFP variants like rsEGFP2 and Dronpa M159T enabled the acquisition of single RESOLFT recordings within seconds [44], [45]. This speed improvement allowed the recording of cellular dynamics in high optical resolution in a reasonable time scale using RESOLFT-microscopy [45], [89], [53], [59]. So far, no high-resolution time-lapse imaging of cellular dynamics in plant cells has been reported. As demonstrated in Figure 2.18, time-lapse RESOLFT imaging of microtubule filaments in leaf epidermis cells is possible. The nearly absence of bleaching during the imaging enabled time-lapse recordings with $\sim 70 \mathrm{~nm}$ resolution in the RESOLFT mode (Figure 2.18). The presented RESOLFT time-lapse recordings in this work are the first demonstration of high-resolution time-lapse imaging in plant cells.

As demonstrated in Section 2.1.5, the RESOLFT parameters can be optimized to increase the imaging speed by applying higher light intensities for the off-switching. For the acquisition of single RESOLFT frames in Arabidopsis thaliana, the offswitching doughnut shaped beam was applied for $480 \mu \mathrm{s}$ at $32 \mu \mathrm{W}$ (Figure 2.15). For fast time-lapse RESOLFT recordings, the off-switching was shortened to $120 \mu \mathrm{s}$ using $43 \mu \mathrm{W}$ to reduce the acquisition time per single RESOLFT frame, thus enable the recording of the dynamics of the microtubule network. As demonstrated by the line profiles in Figure 2.18, the shortening of the off-switching time did not result in a reduction of the achieved optical resolution compared to single RESOLFT recordings. This indicated that the shortening of the off-switching step was compensated by the increased light intensity of the off-switching beam.

One drawback of point scanning RESOLFT-microscopy imaging is the time consuming point-wise switching of the RSFPs at each scanning position. Using the fastest RSFP variants, the recording time of a single RESOLFT frame is slower compared to single STED frames. The dwell time in the performed RESOLFT time-lapse recordings of MAP4-rsEGFP2 labelled tubulin filaments was $180 \mu$ s (Figure 2.18); 
in the performed STED time-lapse imaging using rsEGFP2, it was $59 \mu \mathrm{s}$. STEDmicroscopy on fluorescent proteins is normally performed with non-switchable fluorescent proteins. In the performed STED imaging of MAP4-rsEGFP2 an additional activation step of $20 \mu \mathrm{s}$ was performed at each scan position. Typically, the dwell times of STED-microscopy using non-switchable fluorescent proteins are in the range of $20-50 \mu \mathrm{s}$ [88], [107].

The acquisition time of a single RESOLFT image is dependent on the switching speed of the RSFP used which is typically in the $\mu$ s time range for fast switching RSFPs like rsEGFP2 or DronpaM159T using light intensities of tens of $\mathrm{kW} / \mathrm{cm}^{2}$ [44], [45]. The switching in STED-microscopy can be seen as instantaneous, since it occurs within the fluorescence lifetime of the fluorophore used. Therefore STED recordings are faster as compared to single RESOLFT recordings even if the same fluorescent protein is used. However, as mentioned before, the light intensities used in STED-microscopy are in the $\mathrm{MW} / \mathrm{cm}^{2}$ to $\mathrm{GW} / \mathrm{cm}^{2}$ range, whereas in RESOLFTmicroscopy intensities of only several $\mathrm{kW} / \mathrm{cm}^{2}$ are used.

\subsubsection{FRAP measurements of MAP4-rsEGFP2 labelled microtubules}

Within the performed time-lapse imaging of 40 consecutive RESOLFT frames of MAP4-rsEGFP2 labelled microtuble filaments in leaf epidermis cells, no signs of bleaching were visible (Figure 2.18). The absence of bleaching of MAP4-rsEGFP2 is in contrast to the observed bleaching of rsEGFP2-labelled $\alpha$-tubulin in the recorded time-lapse series of 40 consecutive RESOLFT frames of Drosophila melanogaster (Figure 2.8).

The performed FRAP measurements in leaf epidermis cells demonstrated a fast replacement of the bleached MAP4-rsEGFP2 fusion proteins within $\sim 5$ seconds to recover $50 \%$ of the initial fluorescence.

This replacement is possible, since the microtubule filaments were indirectly labelled via the attachment of a microtubule binding protein fused with rsEGFP2 which enables dissociation and replacement of the bleached rsEGFP2 proteins. In Drosophila melanogaster the microtubules were labelled via expression of rsEGFP2- $\alpha$-tubulin fusion proteins, which were integrated in mictotubule filaments and thus can not be easily replaced.

The fast replacement of MAP4-rsEGFP2 enabled long-term RESOLFT recordings with 250 consecutive RESOLFT frames while the fluorescence signal was only reduced by $\sim 30 \%$. As can be seen in Section 2.17, the fast replacement of bleached MAP4-rsEGFP2 enabled 40 consecutive STED frames without strong 
bleaching of the rsEGFP2 signal. Normally, if the fluorescent proteins cannot be replaced during imaging, only $\sim 10$ consecutive STED frames were possible [107].

\subsubsection{Conclusions and Outlook}

Previous demonstration of live cell high resolution microscopy were restricted to non-photosynthesis active cells or to imaging at the apical cell membrane.

With this work, the applicability of RESOLFT-microscopy in plant cells was characterised and demonstrated for the first time.

It could be shown that RESOLFT-microscopy is capable of high-resolution imaging in green leaf epidermis cells, featuring a resolution of $\sim 60 \mathrm{~nm}-70 \mathrm{~nm}$. Next to the acquisition of single RESOLFT images, time-lapse recordings of living plant cells with $\sim 70 \mathrm{~nm}$ optical resolution could be recorded using RESOLFT-microscopy for the first time.

The implementation of RESOLFT-microscopy using a point scanning microscope enabled imaging beyond the apical cell wall deep within plant tissue with good signal to noise ratio in the recorded high resolution-images. The low light levels applied in RESOLFT-microscopy enabled time-lapse recordings of $1 \mathrm{~h}$ while capturing cellular dynamics in light absorbing leaf epidermis cells.

Based on the performed RESOLFT imaging in this work, high resolution RESOLFT imaging can now be applied to other target structures or proteins of interest to study their localization with high optical resolution in living plant cells. 


\section{Material and Methods}

\subsection{Generation of rsEGFP2- $\alpha$-tubulin expressing Drosophila melanogaster}

For labelling of the microstubule network within Drosophila melanogaster, a synthetic gene sequence encoding the fluorescent protein rsEGFP2 (GenBank \# AGE84598.1) attached to the N-terminus of the alpha-tubulin 84B (CG1913, UniProt \#P06603) protein was synthesized. The synthesised DNA was cloned into an attB-containing fly transformation vector under the control of the Ubiquitin p63E (CG11624) promotor-region (3L; 3901762 to 3903743) using the SpeI and XbaI restriction enzymes (New England Biolabs Inc.). To generate transgenic Drosophila melanogaster flies, a standard phiC31 integrase based germ line transformation procedure was followed, targeting the landing site M3xP3-RFP.attPZH$86 \mathrm{Fb}$ using Bloomington stock \#24749. The injection into the Bloomington stock \#24749 was performed by BestGene Inc. (California, USA). The stock was crossed homozygous and used for all experiments described in Section 2.1.

\subsection{Sample preparation of Drosophila melanogaster}

Third instar larvae of rsEGFP2- $\alpha$-tubulin expressing Drosophila melanogaster were dissected in Schneider's cell culture medium (Life Technologies, California, USA) supplemented with $10 \%$ Fetal Bovine Serum (FBS) to isolate single tissues. The tissues were mounted in Schneider's cell culture medium supplemented with $10 \%$ FBS on a microscope slide and covered with a coverslip (No. $1.5 \mathrm{H} 12 \mathrm{~mm} \varnothing$, Paul Marienfeld GmbH, Lauda-Königshofen, Germany). For in vivo RESOLFT imaging an intact second instar larva was placed between two spacers in Schneider's medium and covered with a coverslip. All samples were sealed using nontoxic duplicating silicone (Picodent, Wipperfuerth, Germany).

\subsection{Western Blot}

To gather protein lysate of rsEGFP2- $\alpha$-tubulin expressing Drosophila melanogaster, wandering third instar larvae were dissected in $1 \mathrm{x}$ PBS to remove the fat tissue and subsequently homogenized in protein extraction buffer $(10 \%$ (v/v) Glycerol, $50 \mathrm{mM}$ HEPES (pH 7.5), 150 mM NaCl, 0.5 \% Triton-X-100, 1.5 mM MgCl, 1 mM EGTA) containing protease inhibitors (Pierce Protease Inhibitor Tablets, ThermoFisher, 
Massachusetts, USA). Protein lysate was centrifuged to pellet cellular debris. The supernatant was transferred to a new $1.5 \mathrm{~mL}$ tube and mixed with Laemmli Buffer (Sigma-Aldrich, St. Louis, Missouri, USA) and denatured for $10 \mathrm{~min}$ at $95{ }^{\circ} \mathrm{C}$ before loading onto an $10 \%$ SDS-polyacrylamide gel. The protein-lysate of approximately two larvae was loaded into each lane of the gel. The SDS gel was blotted using a semidry blotter onto a PVDF membrane. The protein-blot was blocked using $5 \%$ fat free milk powder dissolved in 1x PBS for 1 hour. The polyclonal a-tubulin antiserum (Abcam, Cambridge, England) and the anti-GFP antibody (Clonetech) were used in a concentration of 1:5000 in 2.5\% fat free milk powder dissolved in 1x PBS for 1 hour. HRP-conjugated secondary antibody (Jackson ImmunoResearch, Suffolk, UK) was used at a dilution of 1:10.000 in 2.5\% fat free milk powder dissolved in $1 \mathrm{x}$ PBS for 1 hour and detected with an ECL-kit (Perkin Elmer Life Science, Massachusetts, USA) using a custom built imaging system with a CCD-camera.

\subsection{Western Blot analyis}

To determine the signal ratio of rsEGFP2-labelled $\alpha$-tubulin to non-labelled $\alpha$ tubulin in Western blots, the signal intensities were determined using the ImageJ Plugin Gel Analyzer and the ratio was calculated based on the integral of each respective signal.

\subsection{Lifespan analysis}

The lifespan analysis was performed similar as described before [108]. For the analysis, 510 flies of the same gender were used in 17 vials (30 flies each), the flies were transferred to a new vial every three days and the total number of dead flies was recorded. The data of the lifespan analysis is depicted in Section 5.1.

\subsection{MAP4-rsEGFP2 Plasmid Construction}

To generate the microtubule marker fusion construct rsEGFP2-MAP4, the binary vector pC2 that confers in planta Basta resistance was used [109]. The UBQ10 promoter was integrated into $\mathrm{pC} 2$ via the RsrII restriction sites and the resulting plasmid was linearized with the BamHI and SpeI restriction sites. The rsEGFP2 and MAP4 were generated by PCR amplification from pMD-Vim-rsEGFP2 [45] and pC3 [109] using the oligonucleotide primer pairs CTGATTAACAGCGGTCCGGGATCCATGGTGAGCAAGGGCGAGG and CATCACCGCGTGCTTGTACAGCTCGTCCATGC, and ACAAGCACGCGGT- 
GATGTCCCGGCAAGAAGAAGCAAAG and GGTGATTTTTGCGGACTCTAGACTAGTTTAACCTCCTGCAGGAAAGTGGC, respectively, and ligated with the linearized vector by Gibson Assembly kit (NEB, Hitchin, UK,[110]) to construct the expression vector pUBQ10-rsEGFP2-MAP4.

\subsection{Generation of MAP4-rsEGFP2 expressing Arabidopsis thaliana.}

Seeds of Arabidopsis thaliana ecotype Col-0 were sown onto $8 \mathrm{~cm}^{2}$ pots and vernalized at $4{ }^{\circ} \mathrm{C}$ for three days. For Agrobacterium-mediated transformation, the plants were grown in a climate chamber (Johnson Controls, USA) under long day conditions $(16 / 8 \mathrm{~h})$ at $22 / 18^{\circ} \mathrm{C}$.

\subsection{Arabidopsis thaliana transformation}

Arabidopsis transformation was performed according to the floral dip method [111]. The pUBQ10-MAP4-rsEGFP2 plasmid was transformed into the Agrobacterium tumefaciens strain GV3101 pMP90RK. The resulting Agrobacterium strain were inoculated in LB liquid medium supplemented with $50 \mathrm{mg} / \mathrm{l}$ rifampicin, $30 \mathrm{mg} / \mathrm{l}$ gentamicin, $50 \mathrm{mg} / \mathrm{l} \mathrm{kanamycin,} 50 \mathrm{mg} / \mathrm{l}$ carbenicillin and grown for two days at $28^{\circ} \mathrm{C}$. The suspension culture was centrifuged at $4000 \mathrm{x} g$ for $20 \mathrm{~min}$ and then resuspended in infiltration medium (0.5x MS, $5 \%$ sucrose, and $0.015 \%$ Silwet L-77) that was used for floral dipping. Positive transformants were selected on soil by spraying seedlings of the T1 generation with 0.1\% BASTA (Bayer CropScience, Leverkusen, Germany). Plants of the T4 generation were used for microscopy.

\subsection{Sample preparation}

For imaging of leaf epidermis cells, small pieces of the leaf were cut out using a sharp knife. These pieces were placed in a drop of PBS, covered with a coverslip (Menzel $22 \times 40 \mathrm{~mm}, 1.5 \mathrm{H}$ ) and sealed using non-toxic duplicating silicone (Picodent, Wipperfuerth, Germany). For illustration of the sample preparation see Figure 5.3.

\subsection{RESOLFT-microscopy}

RESOLFT nanoscopy was performed using a modified 1C RESOLFT QUAD Scanning microscope (Abberior Instruments, Göttingen, Germany) equipped with an Olympus UPLSAPO 1.4 NA 100x oil or UPLSAPO 1.3 NA 60x silicone objective. 
For RESOLFT imaging with rsEGFP2 the following switching scheme was applied to the sample at each scanning position: First, illumination with light of $405 \mathrm{~nm}$ wavelength to switch all rsEGFP2 proteins into the fluorescent on-state. Second, off-switching of rsEGFP2 proteins in the periphery of the excitation spot using a $488 \mathrm{~nm}$ doughnut-shaped beam. The 2D-doughnut was realized by using a phase plate, whereas the 3D-doughnut was generated by using a spatial light modulator. Third, the residual on-state fluorophores at the very center of the excitation spot were read out using a Gaussian shaped beam of $488 \mathrm{~nm}$ light. The corresponding confocal images were recorded by applying the same switching scheme as used for RESOLFT imaging without the illumination step using a doughnut shaped beam. Some RESOLFT images were recorded with a line accumulation depending on the signal intensity.

\subsection{STED-microscopy}

STED imaging of MAP4-rsEGFP2 labelled microtubule filaments in Arabidopsis thaliana was performed using a custom build STED microscope which has been described previously [107]. For STED-imaging of MAP4-rsEGFP2, the following imaging scheme was applied to the sample at each scanning position: First, rsEGFP2 proteins were switched into the on-state with light of $405 \mathrm{~nm}$ wavelength. Second, rsEGFP2 proteins were excited using a pulsed $485 \mathrm{~nm}$ diode while the periphery of the focal spot was destimulated using superimposed doughnut-shaped STED beam of $587 \mathrm{~nm}$ (using a $20 \mathrm{MHz}$ repetition rate). The corresponding confocal images were recorded by applying the same switching scheme as used for STED imaging without the illumination with the STED beam.

\subsection{FRAP measurements}

FRAP measurements in upper leaf epidermis cells of Arabidopsis thaliana were performed using a Leica SP8 microscope equipped with a 60x 1.3 NA oil objective. Bleaching of region of interest was performed using simultaneous illumination with $405 \mathrm{~nm}$ and $488 \mathrm{~nm}$ light for 12 seconds. Subsequently, a confocal overview timelapse series of $31 \mu \mathrm{m}$ x $31 \mu \mathrm{m}$ was recorded using simultaneously illumination with $405 \mathrm{~nm}$ and $488 \mathrm{~nm}$ with a pixel dwell time of $4.88 \mu \mathrm{s}$. Recorded time series: 512 x 512 pixel, $4.88 \mu$ s dwell time, recording time per frame: 1.3 seconds. Analysis of the average fluorescence signal in the bleached and non-bleached region in the recorded time-lapse series was performed with Matlab 2016 (The MathWorks, Inc., MA, USA) applying a custom written algorithm described in Section 4.14. 


\subsection{Image analysis - Line profiles}

For determination of the FWHM of single tubulin filaments in recorded images, line profiles averaged along several pixel sizes were measured on raw data and fitted by a standard Lorentz-fit using the software Origin Pro 9.1 (OriginLab, Massachusetts, USA).

\subsection{Image analysis - Filament detection and counting}

To detect the tubulin filaments in the image, we used a ridge detection algorithm based on the calculation of the major eigenvalue of the Hessian Matrix at each pixel of the image [112]. In the resulting image of ridges, locally maximal lines were found by applying a filter returning one if a pixel was among the three largest pixels of it's eight neighbours. The filament brightness was estimated from the smoothed image at the positions of the detected filaments. The median of the brightness values was used to estimate the single filament brightness. The number of filaments was found by dividing the image by the single filament brightness and rounding the result. All procedures were implemented in Matlab 2016 (The MathWorks, Inc., MA, USA).

\subsection{Image manipulations}

All fluorescence images presented in this work are raw data, except where indicated, smoothing by convolution with a 1.2 pixel wide Gaussian using ImSpector (www.imspector.de) was performed. Image manipulations like background subtraction was never performed. False color coding was performed using the Temporal-Color Code plugin of ImageJ. 


\section{Appendix}

\subsection{Determination of average life span}

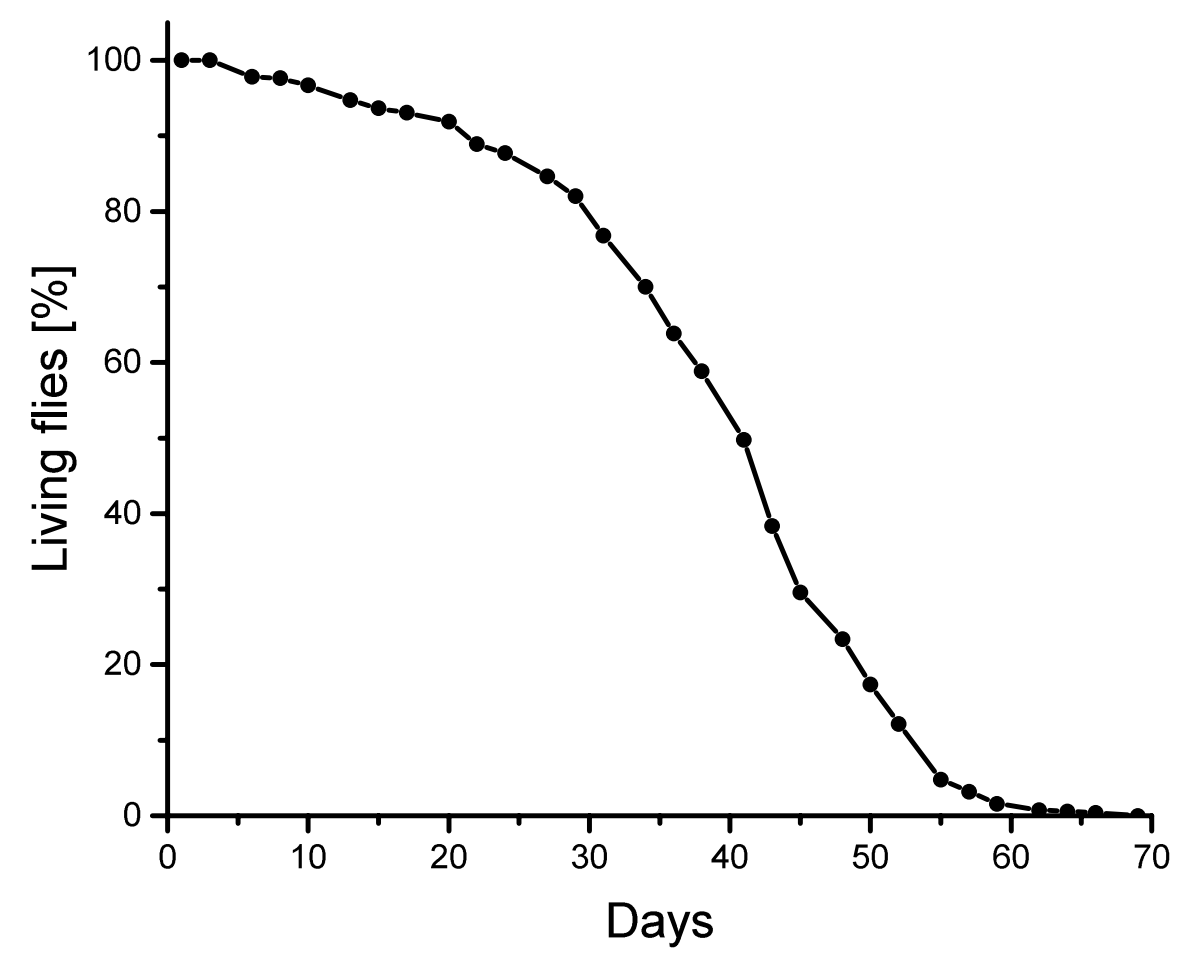

Figure 5.1: Analysis of average life span of rsEGFP2- $\alpha$-tubulin expressing Drosophila melanogaster used in Section 2.1. The flies displayed an average life span of 41 days. Life span analysis was performed as described in Section 4.5. 


\subsection{Western Blot analysis}

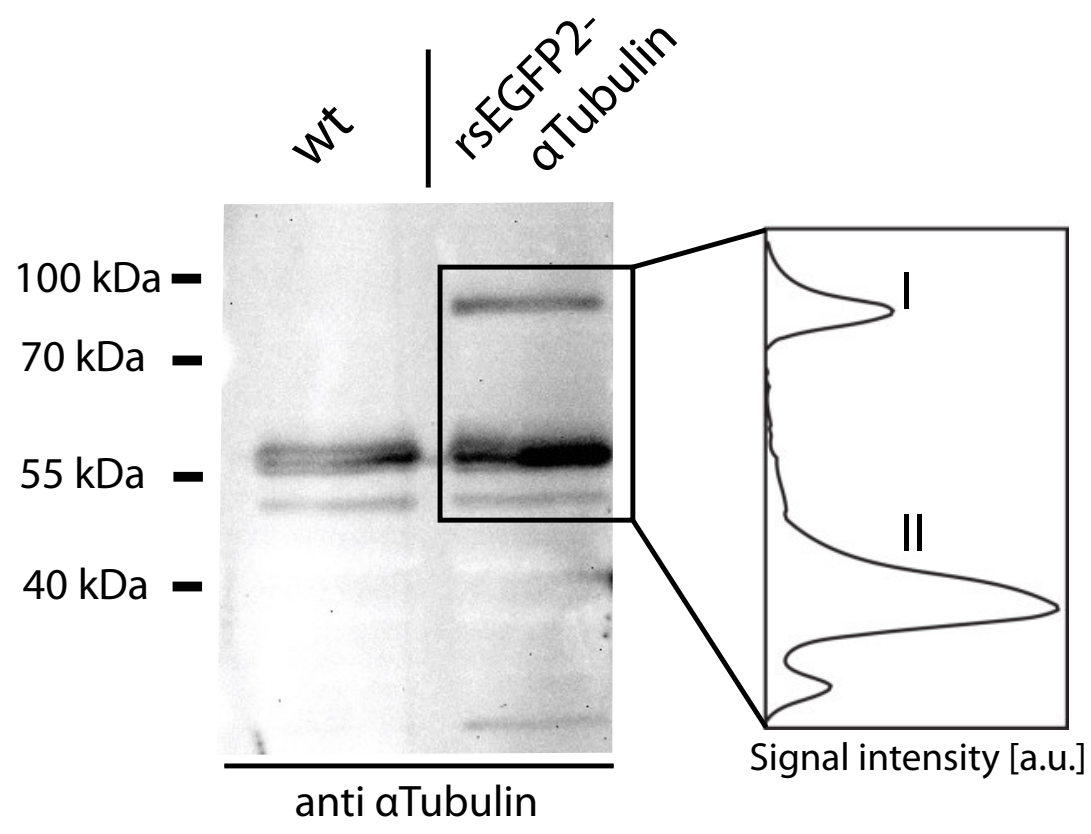

Figure 5.2: Western blot analysis of the expression level of rsEGFP2- $\alpha$ tubulin in Drosophila melanogaster third instar larvae.

To analyse the ratio between rsEGFP2-labelled (peak I) and non-labelled (peak II) $\alpha$-tubulin signals in the Western blot, the respective signals were determined using the ImageJ plugin Gel Analyzer (Figure 5.2). The integral of the peaks for rsEGFP2-labelled (peak I) and non-labelled (peak II) $\alpha$-tubulin signal was determined and the ratio was calculated (Table 5.1)

Table 5.1: Analysis of signal intensities of rsEGFP2-labelled (peak I) and nonlabelled (peak II) $\alpha$-tubulin signal in Figure 5.2.

\begin{tabular}{lcc}
\hline & Signal intensity [a.u.] & $\begin{array}{c}\text { Percentage of } \\
\text { total protein }\end{array}$ \\
\hline rsEGFP2-labelled- $\alpha$-tubulin (peak I) & 3644 & 26.3 \\
Non-labelled- $\alpha$-tubulin (peak II) & 13862 & 73.7
\end{tabular}




\subsection{Sample preparation Arabidopsis thaliana}

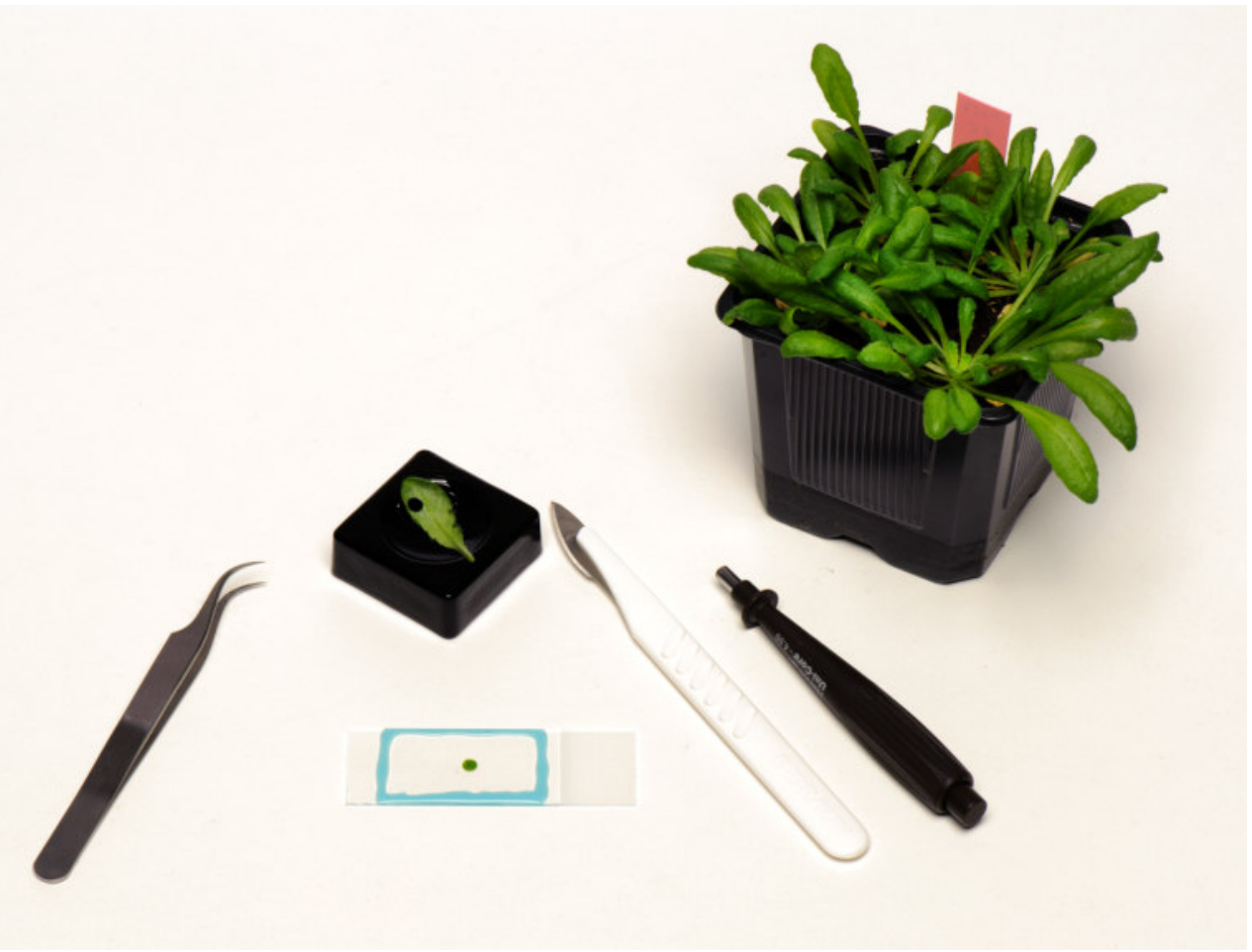

Figure 5.3: Illustration of sample preparation of MAP4-rsEGFP2 expressing Arabidopsis thaliana for imaging of leaf epidermis cells. From green leaves, small pieces of $4 \times 4 \mathrm{~mm}$ size, were cut out and placed in a drop of PBS, covered with a coverslip and sealed using non-toxic duplicating silicone. 


\subsection{References}

1. Hooke, R. Micrographia: or, Some physiological descriptions of minute bodies made by magnifying glasses. (J. Martyn and J. Allestry, 1665).

2. Jabłoński, A. Über den Mechanismus der Photolumineszenz von Farbstoffphosphoren. Zeitschrift für Physik 94, 38-46. ISSN: 0044-3328 (1935).

3. Valeur, B. \& Berberan-Santos, M. N. Molecular fluorescence : principles and applications 2. ed. ISBN: 3527328378 (Wiley-VCH, Weinheim, 2012).

4. Fornasiero, E. F. \& Rizzoli, S. O. Super-resolution microscopy techniques in the neurosciences ISBN: 1627039821 (Humana Press, New York u.a., 2014).

5. Airy, G. On the Diffraction of an Object-glass with Circular Aperture. Transactions of the Cambridge Philosophical Society 5, 283 (1835).

6. Abbe, E. Beiträge zur Theorie des Mikroskops und der mikroskopischen Wahrnehmung. Archiv für Mikroskopische Anatomie 9, 413-468. ISSN: 01767348 (1873).

7. Rayleigh, J. On the theory of optical images, with special reference to the microscope. Philos Mag. 42, 167-195 (1896).

8. Thompson, N. L., Burghardt, T. P. \& Axelrod, D. Measuring surface dynamics of biomolecules by total internal reflection fluorescence with photobleaching recovery or correlation spectroscopy. Biophysical Journal 33, 435-454. ISSN: 0006-3495 1542-0086 (1981).

9. Hell, S. W. Microscopy and its focal switch. Nat Methods 6, 24-32. ISSN: 1548-7091 (2009).

10. Heisenberg, W. The physical principles of the quantum theory (Chicago Univ Press, Chicago, 1930).

11. Van de Linde, S., Loschberger, A., Klein, T., Heidbreder, M., Wolter, S., Heilemann, M. \& Sauer, M. Direct stochastic optical reconstruction microscopy with standard fluorescent probes. Nat. Protocols 6, 991-1009. ISSN: 1754-2189 (2011).

12. Hu, D., Zhao, B., Xie, Y., Orr, G. \& Li, A. D. Understanding super-resolution nanoscopy and its biological applications in cell imaging. Phys Chem Chem Phys 15, 14856-61. ISSN: 1463-9076 (2013). 
13. Betzig, E., Patterson, G. H., Sougrat, R., Lindwasser, O. W., Olenych, S., Bonifacino, J. S., Davidson, M. W., Lippincott-Schwartz, J. \& Hess, H. F. Imaging Intracellular Fluorescent Proteins at Nanometer Resolution. Science 313, 1642-1645. ISSN: 0036-8075 (2006).

14. Hess, S. T., Girirajan, T. P. K. \& Mason, M. D. Ultra-High Resolution Imaging by Fluorescence Photoactivation Localization Microscopy. Biophysical Journal 91, 4258-4272. ISSN: 0006-3495 (2006).

15. Rust, M. J., Bates, M. \& Zhuang, X. Sub-diffraction-limit imaging by stochastic optical reconstruction microscopy (STORM). Nature Methods 3, 793-795. ISSN: 1548-7091 (2006).

16. Huang, B., Wang, W., Bates, M. \& Zhuang, X. Three-dimensional Superresolution Imaging by Stochastic Optical Reconstruction Microscopy. Science (New York, N.Y.) 319, 810-813. ISSN: 0036-8075 1095-9203 (2008).

17. Hell, S. W., Dyba, M. \& Jakobs, S. Concepts for nanoscale resolution in fluorescence microscopy. Curr Opin Neurobiol 14, 599-609. ISSN: 0959-4388 (Print) 0959-4388 (2004).

18. Hell, S. W. Toward fluorescence nanoscopy. Nat Biotechnol 21, 1347-55. ISSN: 1087-0156 (Print) 1087-0156 (2003).

19. Hell, S. W., Jakobs, S. \& Kastrup, L. Imaging and writing at the nanoscale with focused visible light through saturable optical transitions. Applied Physics A 77, 859-860. ISSN: 1432-0630 (2003).

20. Hofmann, M., Eggeling, C., Jakobs, S. \& Hell, S. W. Breaking the diffraction barrier in fluorescence microscopy at low light intensities by using reversibly photoswitchable proteins. Proc Natl Acad Sci U S A 102, 17565-9. ISSN: 0027-8424 (Print) 0027-8424 (2005).

21. Hell, S. W. \& Wichmann, J. Breaking the diffraction resolution limit by stimulated emission: stimulated-emission-depletion fluorescence microscopy. Opt Lett 19, 780-2. ISSN: 0146-9592 (Print) 0146-9592 (1994).

22. Klar, T. A., Jakobs, S., Dyba, M., Egner, A. \& Hell, S. W. Fluorescence microscopy with diffraction resolution barrier broken by stimulated emission. Proceedings of the National Academy of Sciences 97, 8206-8210 (2000).

23. Berezin, M. Y. \& Achilefu, S. Fluorescence Lifetime Measurements and Biological Imaging. Chemical reviews 110, 2641-2684. ISSN: 0009-2665 1520-6890 (2010). 
24. Hell, S. W. \& Kroug, M. Ground-state-depletion fluorscence microscopy: A concept for breaking the diffraction resolution limit. Applied Physics B 60, 495-497. ISSN: 1432-0649 (1995).

25. Donnert, G., Keller, J., Medda, R., Andrei, M. A., Rizzoli, S. O., Luhrmann, R., Jahn, R., Eggeling, C. \& Hell, S. W. Macromolecular-scale resolution in biological fluorescence microscopy. Proc Natl Acad Sci U S A 103, 11440-5. ISSN: 0027-8424 (Print) 0027-8424 (2006).

26. Andresen, M. et al. Structure and mechanism of the reversible photoswitch of a fluorescent protein. Proc Natl Acad Sci U S A 102, 13070-4. ISSN: 0027-8424 (Print) 0027-8424 (2005).

27. Gustafsson, M. G. Nonlinear structured-illumination microscopy: wide-field fluorescence imaging with theoretically unlimited resolution. Proc Natl Acad Sci U S A 102, 13081-6. ISSN: 0027-8424 (Print) 0027-8424 (2005).

28. Li, D. et al. Extended-resolution structured illumination imaging of endocytic and cytoskeletal dynamics. Science 349, aab3500. ISSN: 1095-9203 (Electronic) (2015).

29. Shimomura, O., Johnson, F. H. \& Saiga, Y. Extraction, Purification and Properties of Aequorin, a Bioluminescent Protein from the Luminous Hydromedusan, Aequorea. Journal of Cellular and Comparative Physiology 59, 223-239 (1962).

30. Chalfie, M., Tu, Y., Euskirchen, G., Ward, W. W. \& Prasher, D. C. Green fluorescent protein as a marker for gene expression. Science 263, 802-5. ISSN: 0036-8075 (Print) 0036-8075 (1994).

31. Ormo, M., Cubitt, A. B., Kallio, K., Gross, L. A., Tsien, R. Y. \& Remington, S. J. Crystal structure of the Aequorea victoria green fluorescent protein. Science 273, 1392-5. ISSN: 0036-8075 (Print) 0036-8075 (1996).

32. Heim, R., Prasher, D. C. \& Tsien, R. Y. Wavelength mutations and posttranslational autoxidation of green fluorescent protein. Proc Natl Acad Sci U S A 91, 12501-4. ISSN: 0027-8424 (Print) 0027-8424 (1994).

33. Niwa, H., Inouye, S., Hirano, T., Matsuno, T., Kojima, S., Kubota, M., Ohashi, M. \& Tsuji, F. I. Chemical nature of the light emitter of the Aequorea green fluorescent protein. Proc Natl Acad Sci U S A 93, 13617-22. ISSN: 0027-8424 (Print) 0027-8424 (1996). 
34. Brejc, K., Sixma, T. K., Kitts, P. A., Kain, S. R., Tsien, R. Y., Ormo, M. \& Remington, S. J. Structural basis for dual excitation and photoisomerization of the Aequorea victoria green fluorescent protein. Proc Natl Acad Sci U S A 94, 2306-11. ISSN: 0027-8424 (Print) 0027-8424 (1997).

35. Heim, R., Cubitt, A. B. \& Tsien, R. Y. Improved green fluorescence. Nature 373, 663-4. ISSN: 0028-0836 (Print) 0028-0836 (1995).

36. Lukyanov, K. A. et al. Natural animal coloration can Be determined by a nonfluorescent green fluorescent protein homolog. J Biol Chem 275, 2587982. ISSN: 0021-9258 (2000).

37. Ando, R., Mizuno, H. \& Miyawaki, A. Regulated Fast Nucleocytoplasmic Shuttling Observed by Reversible Protein Highlighting. Science 306, 13701373 (2004).

38. Andresen, M., Stiel, A. C., Trowitzsch, S., Weber, G., Eggeling, C., Wahl, M. C., Hell, S. W. \& Jakobs, S. Structural basis for reversible photoswitching in Dronpa. Proc Natl Acad Sci U S A 104, 13005-9. ISSN: 0027-8424 (Print) 0027-8424 (Linking) (2007).

39. Petersen, J., Wilmann, P. G., Beddoe, T., Oakley, A. J., Devenish, R. J., Prescott, M. \& Rossjohn, J. The 2.0-angstrom crystal structure of eqfp611, a far red fluorescent protein from the sea anemone entacmaea quadricolor. Journal of Biological Chemistry 278, 44626-44631. ISSN: 0021-9258 (2003).

40. Merzlyak, E. M. et al. Bright monomeric red fluorescent protein with an extended fluorescence lifetime. Nat Methods 4, 555-7. ISSN: 1548-7091 (Print) 1548-7091 (2007).

41. Brakemann, T. et al. Molecular basis of the light-driven switching of the photochromic fluorescent protein Padron. J Biol Chem 285, 14603-9. ISSN: 0021-9258 (2010).

42. Brakemann, T. et al. A reversibly photoswitchable GFP-like protein with fluorescence excitation decoupled from switching. Nat Biotechnol 29, 942-7 (2011).

43. Lacombat, F., Plaza, P., Plamont, M. A. \& Espagne, A. Photoinduced Chromophore Hydration in the Fluorescent Protein Dreiklang Is Triggered by Ultrafast Excited-State Proton Transfer Coupled to a Low-Frequency Vibration. J Phys Chem Lett 8, 1489-1495. ISSN: 1948-7185 (2017). 
44. Stiel, A. C., Trowitzsch, S., Weber, G., Andresen, M., Eggeling, C., Hell, S. W., Jakobs, S. \& Wahl, M. C. 1.8 A bright-state structure of the reversibly switchable fluorescent protein Dronpa guides the generation of fast switching variants. Biochem J 402, 35-42. ISSN: 0264-6021 (2007).

45. Grotjohann, T., Testa, I., Reuss, M., Brakemann, T., Eggeling, C., Hell, S. W. \& Jakobs, S. rsEGFP2 enables fast RESOLFT nanoscopy of living cells. Elife 1, e00248 (2012).

46. Grotjohann, T., Testa, I., Leutenegger, M., Bock, H., Urban, N. T., LavoieCardinal, F., Willig, K. I., Eggeling, C., Jakobs, S. \& Hell, S. W. Diffractionunlimited all-optical imaging and writing with a photochromic GFP. Nature 478, 204-8 (2011).

47. Andresen, M., Stiel, A. C., Folling, J., Wenzel, D., Schonle, A., Egner, A., Eggeling, C., Hell, S. W. \& Jakobs, S. Photoswitchable fluorescent proteins enable monochromatic multilabel imaging and dual color fluorescence nanoscopy. Nat Biotechnol 26, 1035-40. ISSN: 1546-1696 (Electronic) 10870156 (Linking) (2008).

48. Stiel, A. C., Andresen, M., Bock, H., Hilbert, M., Schilde, J., Schonle, A., Eggeling, C., Egner, A., Hell, S. W. \& Jakobs, S. Generation of monomeric reversibly switchable red fluorescent proteins for far-field fluorescence nanoscopy. Biophys J 95, 2989-97. ISSN: 0006-3495 (2008).

49. Subach, F. V., Zhang, L., Gadella, T. W., Gurskaya, N. G., Lukyanov, K. A. \& Verkhusha, V. V. Red fluorescent protein with reversibly photoswitchable absorbance for photochromic FRET. Chem Biol 17, 745-55. ISSN: 1074-5521 (2010).

50. Chmyrov, A., Keller, J., Grotjohann, T., Ratz, M., d'Este, E., Jakobs, S., Eggeling, C. \& Hell, S. W. Nanoscopy with more than 100,000 'doughnuts'. Nat Methods 10, 737-40. ISSN: 1548-7091 (2013).

51. Wang, S., Chen, X., Chang, L., Xue, R., Duan, H. \& Sun, Y. GMars-Q Enables Long-Term Live-Cell Parallelized Reversible Saturable Optical Fluorescence Transitions Nanoscopy. ACS Nano. ISSN: 1936-0851 (2016).

52. Lavoie-Cardinal, F., Jensen, N. A., Westphal, V., Stiel, A. C., Chmyrov, A., Bierwagen, J., Testa, I., Jakobs, S. \& Hell, S. W. Two-color RESOLFT nanoscopy with green and red fluorescent photochromic proteins. Chemphyschem 15, 655-63. ISSN: 1439-4235 (2014). 
53. Testa, I., D’Este, E., Urban, N. T., Balzarotti, F. \& Hell, S. W. Dual Channel RESOLFT Nanoscopy by Using Fluorescent State Kinetics. Nano Lett. ISSN: 1530-6984 (2014).

54. El Khatib, M., Martins, A., Bourgeois, D., Colletier, J. P. \& Adam, V. Rational design of ultrastable and reversibly photoswitchable fluorescent proteins for super-resolution imaging of the bacterial periplasm. Sci Rep 6, 18459. ISSN: 2045-2322 (2016).

55. Duwe, S., De Zitter, E., Gielen, V., Moeyaert, B., Vandenberg, W., Grotjohann, T., Clays, K., Jakobs, S., Van Meervelt, L. \& Dedecker, P. ExpressionEnhanced Fluorescent Proteins Based on Enhanced Green Fluorescent Protein for Super-resolution Microscopy. ACS Nano 9, 9528-41. ISSN: 1936-0851 (2015).

56. Tiwari, D. K., Arai, Y., Yamanaka, M., Matsuda, T., Agetsuma, M., Nakano, M., Fujita, K. \& Nagai, T. A fast- and positively photoswitchable fluorescent protein for ultralow-laser-power RESOLFT nanoscopy. Nat Methods. ISSN: 1548-7091 (2015).

57. Zhang, X., Zhang, M., Li, D., He, W., Peng, J., Betzig, E. \& Xu, P. Highly photostable, reversibly photoswitchable fluorescent protein with high contrast ratio for live-cell superresolution microscopy. Proc Natl Acad Sci U S A. ISSN: 0027-8424 (2016).

58. Rizzo, M. A., Davidson, M. W. \& Piston, D. W. Fluorescent protein tracking and detection: applications using fluorescent proteins in living cells. Cold Spring Harb Protoc 2009, pdb.top64. ISSN: 1559-6095 (2009).

59. Ratz, M., Testa, I., Hell, S. W. \& Jakobs, S. CRISPR/Cas9-mediated endogenous protein tagging for RESOLFT super-resolution microscopy of living human cells. Scientific Reports 5, 9592 (2015).

60. Wäldchen, S., Lehmann, J., Klein, T., van de Linde, S. \& Sauer, M. Lightinduced cell damage in live-cell super-resolution microscopy. Scientific Reports 5,15348 (2015).

61. Parton, R. M., Valles, A. M., Dobbie, I. M. \& Davis, I. Live cell imaging in Drosophila melanogaster. Cold Spring Harb Protoc 2010, pdb.top75. ISSN: 1559-6095 (2010).

62. Brand, A. H. \& Perrimon, N. Targeted gene expression as a means of altering cell fates and generating dominant phenotypes. Development 118, 401-15. ISSN: 0950-1991 (Print) 0950-1991 (Linking) (1993). 
63. Gibson, T. J., Seiler, M. \& Veitia, R. A. The transience of transient overexpression. Nat Methods 10, 715-21. ISSN: 1548-7091 (2013).

64. Gustafsson, M. G. Surpassing the lateral resolution limit by a factor of two using structured illumination microscopy. J Microsc 198, 82-7. ISSN: 00222720 (Print) 0022-2720 (2000).

65. Kittel, R. J. et al. Bruchpilot promotes active zone assembly, Ca2+ channel clustering, and vesicle release. Science 312, 1051-4. ISSN: 0036-8075 (2006).

66. Fouquet, W., Owald, D., Wichmann, C., Mertel, S., Depner, H., Dyba, M., Hallermann, S., Kittel, R. J., Eimer, S. \& Sigrist, S. J. Maturation of active zone assembly by Drosophila Bruchpilot. The Journal of Cell Biology 186, 129-145 (2009).

67. Owald, D. et al. A Syd-1 homologue regulates pre- and postsynaptic maturation in Drosophila. J Cell Biol 188, 565-79. ISsN: 0021-9525 (2010).

68. Schneider, J., Zahn, J., Maglione, M., Sigrist, S. J., Marquard, J., Chojnacki, J., Krausslich, H.-G., Sahl, S. J., Engelhardt, J. \& Hell, S. W. Ultrafast, temporally stochastic STED nanoscopy of millisecond dynamics. Nat Meth 12, 827-830. ISSN: 1548-7091 (2015).

69. Ehmann, N. et al. Quantitative super-resolution imaging of Bruchpilot distinguishes active zone states. Nat Commun 5, 4650. ISSN: 2041-1723 (2014).

70. Truong Quang, B. A., Mani, M., Markova, O., Lecuit, T. \& Lenne, P. F. Principles of E-cadherin supramolecular organization in vivo. Curr Biol 23, 2197-207. ISSN: 0960-9822 (2013).

71. Wegel, E., Gohler, A., Lagerholm, B. C., Wainman, A., Uphoff, S., Kaufmann, R. \& Dobbie, I. M. Imaging cellular structures in super-resolution with SIM, STED and Localisation Microscopy: A practical comparison. Sci Rep 6, 27290. ISSN: 2045-2322 (2016).

72. Schubert, V. Super-resolution Microscopy - Applications in Plant Cell Research. Frontiers in Plant Science 8. ISSN: 1664-462X (2017).

73. Radotić, K., Kalauzi, A., Djikanović, D., Jeremić, M., Leblanc, R. M. \& Cerović, Z. G. Component analysis of the fluorescence spectra of a lignin model compound. Journal of Photochemistry and Photobiology B: Biology 83, 1-10. ISSN: 1011-1344 (2006). 
74. Liesche, J., Ziomkiewicz, I. \& Schulz, A. Super-resolution imaging with Pontamine Fast Scarlet 4BS enables direct visualization of cellulose orientation and cell connection architecture in onion epidermis cells. BMC Plant Biol 13, 226. ISSN: 1471-2229 (2013).

75. Kleine-Vehn, J. et al. Recycling, clustering, and endocytosis jointly maintain PIN auxin carrier polarity at the plasma membrane. Mol Syst Biol 7, 540. ISSN: 1744-4292 (2011).

76. Komis, G., Šamajová, O., Ovečka, M. \& Šamaj, J. Super-resolution Microscopy in Plant Cell Imaging. Trends in Plant Science 20, 834-843. ISSN: 1360-1385 (2015).

77. Schnorrenberg, S., Grotjohann, T., Vorbruggen, G., Herzig, A., Hell, S. W. \& Jakobs, S. In vivo super-resolution RESOLFT microscopy of Drosophila melanogaster. Elife 5. ISSN: 2050-084x (2016).

78. Marriott, G. et al. Optical lock-in detection imaging microscopy for contrastenhanced imaging in living cells. Proc Natl Acad Sci U S A 105, 17789-94. ISSN: 0027-8424 (2008).

79. Yao, J., Shcherbakova, D. M., Li, C., Krumholz, A., Lorca, R. A., Reinl, E., England, S. K., Verkhusha, V. V. \& Wang, L. V. Reversibly switchable fluorescence microscopy with enhanced resolution and image contrast. J Biomed Opt 19, 086018. ISSN: 1083-3668 (2014).

80. Marc, J., Granger, C. L., Brincat, J., Fisher, D. D., Kao, T., McCubbin, A. G. \& Cyr, R. J. A GFP-MAP4 reporter gene for visualizing cortical microtubule rearrangements in living epidermal cells. Plant Cell 10, 1927-40 (1998).

81. Grefen, C., Donald, N., Hashimoto, K., Kudla, J., Schumacher, K. \& Blatt, M. R. A ubiquitin-10 promoter-based vector set for fluorescent protein tagging facilitates temporal stability and native protein distribution in transient and stable expression studies. The Plant Journal 64, 355-365. ISSN: 1365-313X (2010).

82. Krause, G. H. \& Weis, E. Chlorophyll Fluorescence and Photosynthesis: The Basics. Annual Review of Plant Physiology and Plant Molecular Biology 42, 313-349 (1991).

83. Amos, L. \& Klug, A. Arrangement of subunits in flagellar microtubules. $J$ Cell Sci 14, 523-49. ISSN: 0021-9533 (Print) 0021-9533 (1974). 
84. Cohen, R. N., van der Aa, M. A. E. M., Macaraeg, N., Lee, A. P. \& Szoka, F. C. Quantification of Plasmid DNA Copies in the Nucleus after Lipoplex and Polyplex Transfection. Journal of controlled release 135, 166-174. ISSN: 0168-3659 (2009).

85. Gratz, S. J., Cummings, A. M., Nguyen, J. N., Hamm, D. C., Donohue, L. K., Harrison, M. M., Wildonger, J. \& O'Connor-Giles, K. M. Genome engineering of Drosophila with the CRISPR RNA-guided Cas9 nuclease. Genetics 194, 1029-35 (2013).

86. Gratz, S. J., Ukken, F. P., Rubinstein, C. D., Thiede, G., Donohue, L. K., Cummings, A. M. \& O'Connor-Giles, K. M. Highly specific and efficient CRISPR/Cas9-catalyzed homology-directed repair in Drosophila. Genetics 196, 961-71. ISSN: 0016-6731 (2014).

87. Bassett, A. R., Tibbit, C., Ponting, C. P. \& Liu, J. L. Mutagenesis and homologous recombination in Drosophila cell lines using CRISPR/Cas9. Biol Open 3, 42-9 (2014).

88. Hein, B., Willig, K. I. \& Hell, S. W. Stimulated emission depletion (STED) nanoscopy of a fluorescent protein-labeled organelle inside a living cell. Proceedings of the National Academy of Sciences 105, 14271-14276 (2008).

89. Testa, I., Urban, N. T., Jakobs, S., Eggeling, C., Willig, K. I. \& Hell, S. W. Nanoscopy of living brain slices with low light levels. Neuron 75, 992-1000. ISSN: 0896-6273 (2012).

90. Stelzer, E. H. Light-sheet fluorescence microscopy for quantitative biology. Nat Methods 12, 23-6. ISSN: 1548-7091 (2015).

91. Wagner, M., Weber, P., Bruns, T., Strauss, W. S., Wittig, R. \& Schneckenburger, H. Light dose is a limiting factor to maintain cell viability in fluorescence microscopy and single molecule detection. Int J Mol Sci 11, 956-66. ISSN: 1422-0067 (2010).

92. Bohm, U., Hell, S. W. \& Schmidt, R. 4Pi-RESOLFT nanoscopy. Nat Commun 7, 10504. ISSN: 2041-1723 (2016).

93. Riedl, J. et al. Lifeact: a versatile marker to visualize F-actin. Nat Methods 5, 605-7. ISSN: 1548-7091 (2008).

94. Harris, K. M. \& Kater, S. B. Dendritic spines: cellular specializations imparting both stability and flexibility to synaptic function. Annu Rev Neurosci 17, 341-71 (1994). 
95. Ghannad Rezaie, M., Wang, X., Mishra, B., Collins, C. \& Chronis, N. Microfluidic Chips for In Vivo Imaging of Cellular Responses to Neural Injury in Drosophila Larvae. PLOS ONE 7 (2012).

96. Andlauer, T. F. \& Sigrist, S. J. Building an imaging chamber for in vivo imaging of Drosophila larvae. Cold Spring Harb Protoc 2012, 476-80. ISSN: 1559-6095 (2012).

97. Weissleder, R. A clearer vision for in vivo imaging. Nat Biotechnol 19, 316-7. ISSN: 1087-0156 (Print) 1087-0156 (2001).

98. Yu, D. et al. An improved monomeric infrared fluorescent protein for neuronal and tumour brain imaging. Nat Commun 5, 3626. ISSN: 2041-1723 (2014).

99. Yu, D. et al. A naturally-monomeric infrared fluorescent protein for protein labeling in vivo. Nature methods 12, 763-765. ISSN: 1548-7091 1548-7105 (2015).

100. Rego, E. H., Shao, L., Macklin, J. J., Winoto, L., Johansson, G. A., KampsHughes, N., Davidson, M. W. \& Gustafsson, M. G. Nonlinear structuredillumination microscopy with a photoswitchable protein reveals cellular structures at 50-nm resolution. Proc Natl Acad Sci U S A 109, E135-43. ISSN: 0027-8424 (2012).

101. Komis, G., Mistrik, M., Samajova, O., Doskocilova, A., Ovecka, M., Illes, P., Bartek, J. \& Samaj, J. Dynamics and organization of cortical microtubules as revealed by superresolution structured illumination microscopy. Plant Physiol 165, 129-48. ISSN: 0032-0889 (2014).

102. Benfey, P. N. \& Chua, N. H. The Cauliflower Mosaic Virus 35S Promoter: Combinatorial Regulation of Transcription in Plants. Science 250, 959-66. ISSN: 0036-8075 (Print) 0036-8075 (1990).

103. Mishiba, K.-i., Nishihara, M., Nakatsuka, T., Abe, Y., Hirano, H., Yokoi, T., Kikuchi, A. \& Yamamura, S. Consistent transcriptional silencing of 35Sdriven transgenes in gentian. The Plant Journal 44, 541-556. ISSN: 1365-313X (2005).

104. Zhou, X., Carranco, R., Vitha, S. \& Hall, T. C. The dark side of green fluorescent protein. New Phytologist 168, 313-322. ISSN: 1469-8137 (2005).

105. Kodama, Y. Time Gating of Chloroplast Autofluorescence Allows Clearer Fluorescence Imaging In Planta. PLoS ONE 11, e0152484. ISSN: 1932-6203 (2016). 
106. Wu, C., Chiera, J. M., Ling, P. P. \& Finer, J. J. Isoxaflutole treatment leads to reversible tissue bleaching and allows for more effective detection of GFP in transgenic soybean tissues. In Vitro Cellular and Developmental Biology Plant 44, 540-547. ISSN: 1475-2689 (2008).

107. Danzl, J. G., Sidenstein, S. C., Gregor, C., Urban, N. T., Ilgen, P., Jakobs, S. \& Hell, S. W. Coordinate-targeted fluorescence nanoscopy with multiple off states. Nat Photon 10, 122-128. ISSN: 1749-4885 (2016).

108. Linford, N. J., Bilgir, C., Ro, J. \& Pletcher, S. D. Measurement of Lifespan in Drosophila melanogaster. Journal of Visualized Experiments : JoVE, 50068. ISSN: 1940-087X (2013).

109. Ghareeb, H., Laukamm, S. \& Lipka, V. COLORFUL-Circuit: A Platform for Rapid Multigene Assembly, Delivery, and Expression in Plants. Frontiers in Plant Science 7, 246. ISSN: 1664-462X (2016).

110. Gibson, D. G., Young, L., Chuang, R. Y., Venter, J. C., Hutchison C. A., 3. \& Smith, H. O. Enzymatic assembly of DNA molecules up to several hundred kilobases. Nat Methods 6, 343-5. ISSN: 1548-7091 (2009).

111. Clough, S. J. \& Bent, A. F. Floral dip: a simplified method for Agrobacteriummediated transformation of Arabidopsis thaliana. Plant $J$ 16, 735-43. ISSN: 0960-7412 (Print) 0960-7412 (1998).

112. Steger, C. An unbiased detector of curvilinear structures. IEEE Transactions on Pattern Analysis and Machine Intelligence 20, 113-125. ISSN: 0162-8828 (1998). 


\subsection{List of Figures}

\section{List of Figures}

1.1 Jablonski diagram of energetic states of a fluorescent molecule. . . . . 2

1.2 Sketch of a scanning confocal microscope. . . . . . . . . . . . . . 3

1.3 Principle of coordinated-stochastic imaging methods . . . . . . . . . . 7

1.4 Principle of RESOLFT-microscopy . . . . . . . . . . . . . . . . . . . 8

1.5 Crystal structure and photo-switching of Dronpa. . . . . . . . . . . 13

1.6 Switching mechanisms of the RSFP Dronpa. . . . . . . . . . . . . . 14

1.7 Application of rsEGFP and rsEGFP2 in RESOLFT-microscopy. . . . 16

1.8 Imaging scheme of RESOLFT-microscopy utilizing negative switching RSFPs . . . . . . . . . . . . . . . . . 19

2.1 Confocal recordings of living resected tissues of transgenic wandering third instar larvae and of adult flies ubiquitously expressing rsEGFP2$\alpha$-tubulin. . . . . . . . . . . . . . . 2 25

2.2 Western blot analysis of the expression level of rsEGFP2- $\alpha$-tubulin in Drosophila melanogaster third instar larvae. . . . . . . . . . . . . 26

2.3 RESOLFT-microscopy of resected tissues from Drosophila melanogaster. . . . . . . . . . . . . . . . . . . 29

2.4 RESOLFT volume imaging using a xy-doughnut for off-switching. . . 30

2.5 RESOLFT imaging of volumes using a 3D-light pattern for offswitching. . . . . . . . . . . . . . . . . 32

2.6 Image quality of confocal detection in tissues of Drosophila melanogaster. . . . . . . . . . . . . . . 34

2.7 RESOLFT imaging at different imaging depths. . . . . . . . . . . . . 35

2.8 Time-lapse RESOLFT imaging with high frame rate of the microtubule network in body wall muscles of a dissected Drosophila melanogaster larva. . . . . . . . . . . . . . . . . . 37

2.9 Time-lapse RESOLFT imaging of the microtubule cytoskeleton in body wall muscles of dissected Drosophila melanogaster larva . . . . . 38

2.10 In vivo RESOLFT-microscopy of intact Drosophila melanogaster larva. 40

2.11 In vivo-RESOLFT imaging of intact Drosophila melanogaster larva at different scan positions in the larva. . . . . . . . . . . . . . . . 41

2.12 Determination of the achieved lateral image resolution in intact larvae. 42

2.13 Live cell confocal microscopic images of MAP4-rsEGFP2 labelled microtubule filaments in different cell types. . . . . . . . . . . . . . . 44

2.14 Autofluorescence reduction in live cell microscopy via fluorescence lifetime gating. . . . . . . . . . . . . . . . . . . 4 45

2.15 High-resolution RESOLFT imaging of MAP4-rsEGFP2 labelled tubulin filaments in leaf epidermis cells. . . . . . . . . . . . . . . 47

2.16 High-resolution STED imaging of MAP4-rsEGFP2 labelled microtubule filaments. . . . . . . . . . . . . . . . . . . . 48

2.17 High-resolution STED time-lapse imaging of MAP4-rsEGFP2 labelled tubulin filaments in leaf epidermis cells. . . . . . . . . . . . . . 49 
2.18 Time-lapse RESOLFT imaging of MAP4-rsEGFP2 labelled microtubule filaments in upper leaf epidermis cells. . . . . . . . . . . . 51

2.19 Bleaching analysis of time-lapse RESOLFT imaging in leaf epidermis cells. . . . . . . . . . . . . . . . . . . 5 5 53

2.20 FRAP measurement on MAP4-rsEGFP2 labelled microtubules in upper leaf epidermis cells of Arabidopsis thaliana . . . . . . . . . . . . 54

2.21 Filament counting and tracking in time-lapse RESOLFT recordings of the microtubule filaments in leaf epidermis cells. . . . . . . . . 56

5.1 Determination of average life span of rsEGFP2- $\alpha$-tubulin expressing Drosophila melanogaster . . . . . . . . . . . . . . . . . . . . . 78

5.2 Western blot analysis of the expression level of rsEGFP2- $\alpha$-tubulin in Drosophila melanogaster third instar larvae. . . . . . . . . . . . . . 79

5.3 Illustration of sample preparation of MAP4-rsEGFP2 expressing Arabidopsis thaliana for imaging of leaf epidermis cells. . . . . . . . . . . 80 


\subsection{List of Tables}

\section{List of Tables}

5.1 Analysis of signal intensities of rsEGFP2-labelled (peak I) and nonlabelled (peak II) $\alpha$-tubulin signal in Figure 5.2 . . . . . . . . . . . 79 


\subsection{Publications and use of published material}

Parts of this thesis have been published in the following publication:

Schnorrenberg, S., Grotjohann, T., Vorbruggen, G., Herzig, A., Hell, S. W., Jakobs, S., In vivo super-resolution RESOLFT microscopy of Drosophila melanogaster. eLIFE 5, 2016.

From this publication figure material was used in Section 2.1, the original source was cited in accordance with the Creative Commons Attribution license. 


\subsection{Acknowledgements}

I would like to thank all people who accompanied me during my doctoral thesis. Without their support and help, this thesis would not have been possible.

First I want to thank Prof. Dr. Stefan Jakobs for giving me the opportunity to work on this $\mathrm{PhD}$-thesis in his group and for his guidance throughout the 3.5 years. Next, I want to thank my thesis advisor Dr. Halyna Shcherbata for her support and advices during my $\mathrm{PhD}$ thesis which were an great help for this work. I am especially grateful to Dr. Tim Grotjohann for his fantastic supervision, guidance and help. It had been an great time working with him at the microscope. I would like to thank Prof. Dr. Stefan Hell for giving me the opportunity to work in this fantastic environment of the NanoBiophotonics group.

Additionally, I want to thank Dr. Melina Schuh, Dr. Gerd Vorbrüggen and Dr. Thomas Teichmann for being members of the examination board.

I am thankful to Gerd Vorbrüggen for all advices and for answering all my fly related questions throughout this thesis.

Further, I would like to thank Hassan Ghareeb for the generation of the Arabidopsis transformants used in this work and for the steady supply with new seedlings.

A big thank goes to Lars Frahm for his help with the bleaching analysis and the nice coffee breaks on the roof of tower 2. I am thankful to Dr. Jan Keller for programming of the PSF calculator and help with all my Matlab questions.

I want to thank Ellen Rothermel, Sylvia Löbermann and Rita Schmitz-Salue for excellent technical assistance and for keeping the lab running.

I am very thankful to Jaydev Jethwa for proofreading this thesis and for having the right cable when I needed one.

I am thankful to the complete Jäckle Department for the friendly atmosphere and the nice (scientific) conversations in the fly room. Further, I want to thank Dr. Alf Herzig for the cloning strategy and the plasmid pUbi-tubulin-attP used in this work.

I am very thankful to all my colleagues in the NanoBiophotonics Department who answered all my questions and were always a great help during all these years.

I want to thank all the other PhD-students who have become good friends in the last years. I am especially thankful to my office mate Dirk Meineke for the fantastic time together in the office as well as during bouldering in the last years. I am also very thankful to Christian Brüser who is always a good friend and a great help in many ways. Further, I want to thank Philipp Alt, Timo Konen, Isabelle Jansen, Lars Frahm, Jasmin Hubrich, Carola Gregor, Nickels Jensen, Martin Andresen, 
Elke Hebisch, Sven Sidenstein, Jasmin Pape, Klaus Gwosch, Florian Habenstein and many others for the enjoyable time together at work as well as during the free time activities in and around Göttingen.

I would also like to thank Jaydev Jethwa, Dr. Nickels Jensen, Mariah Snyder and Timo Konen for proofreading this thesis.

Finally, I want thank my entire family for all their love and support during these years. 